\title{
Organic light emitting diodes (OLEDs) and OLED-based structurally integrated optical sensors
}

by

\section{Yuankun Cai}

\author{
A dissertation submitted to the graduate faculty \\ in partial fulfillment of the requirements for the degree of \\ DOCTOR OF PHILOSOPHY
}

Major: Condensed Matter Physics

Program of Study Committee:

Joseph Shinar, Major Professor

Vikram Dalal

Rana Biswas

Curt Struck

Edward Yu

Iowa State University

Ames, Iowa

2010 


\section{TABLE OF CONTENTS}

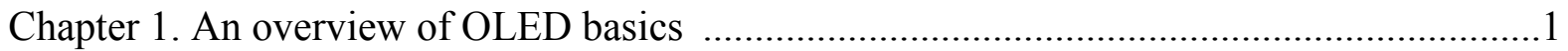

History of organic electroluminescence............................................................

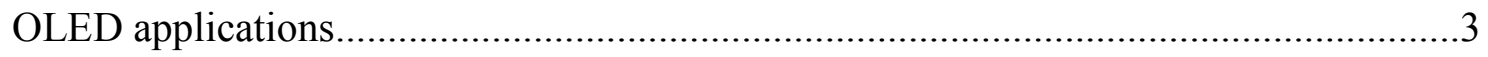

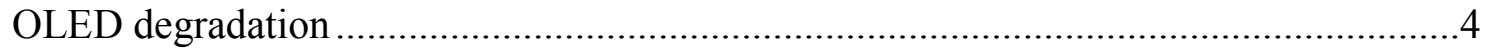

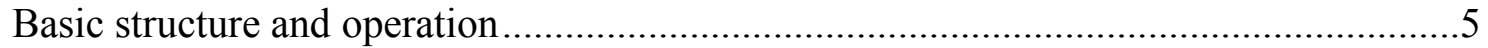

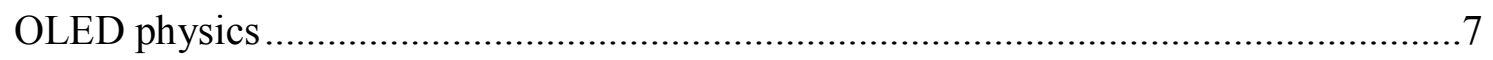

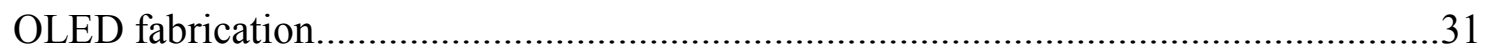

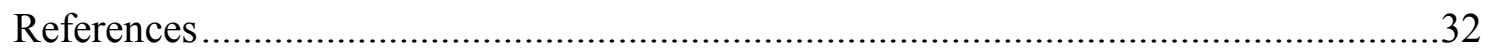

Chapter 2. General introduction to OLED-based structurally integrated optical sensors........36

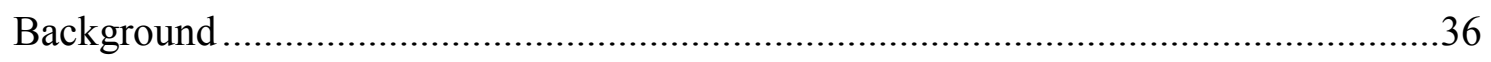

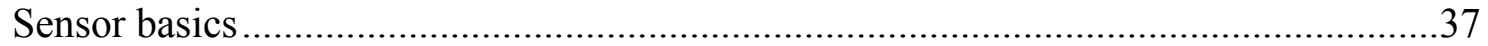

Development of OLED-based structurally integrated sensors...................................42

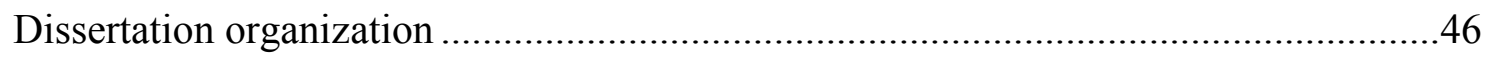

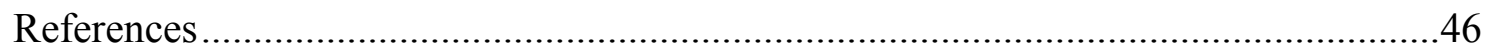

Chapter 3. Interface engineering for OLED improvement .............................................48

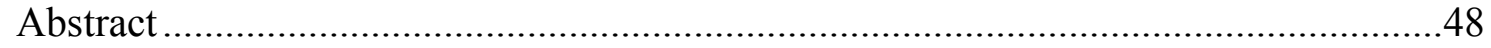

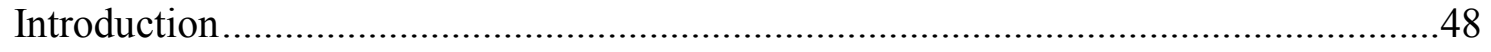

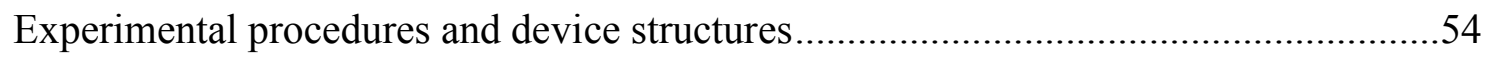

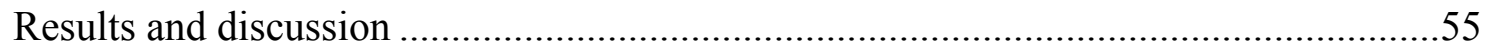

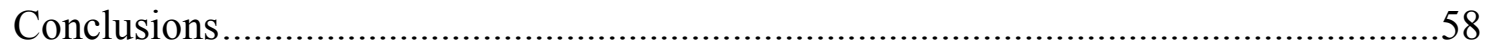

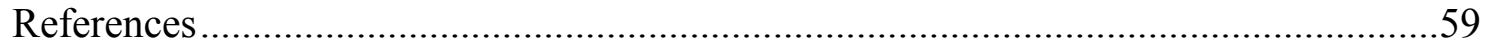

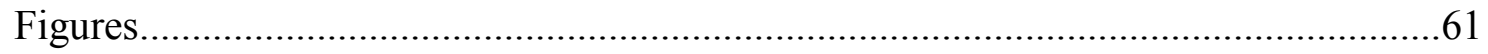

Chapter 4. OLED-based biosensing platform with $\mathrm{ZnO}$ nanoparticles for enzyme immobilization 


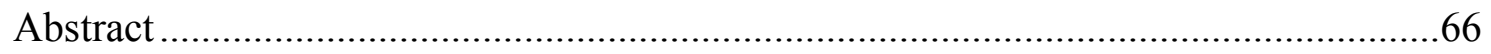

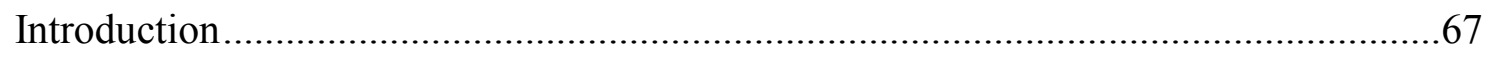

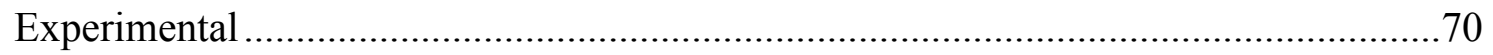

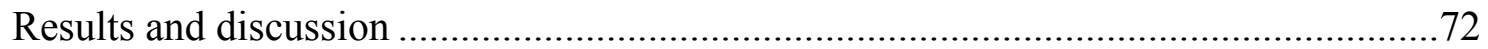

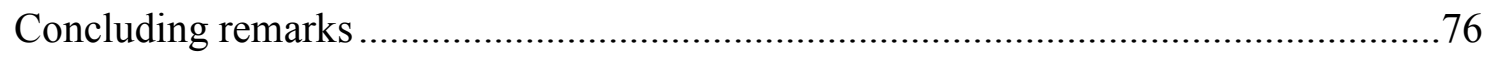

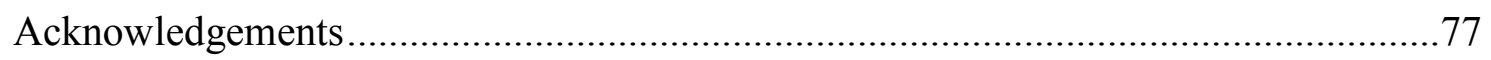

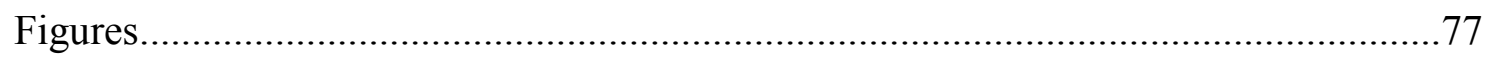

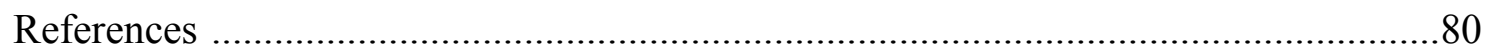

Chapter 5. Multianalyte sensor array based on an organic light emitting diode platform........82

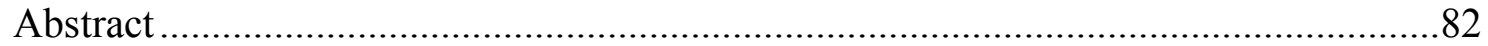

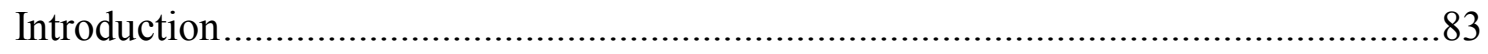

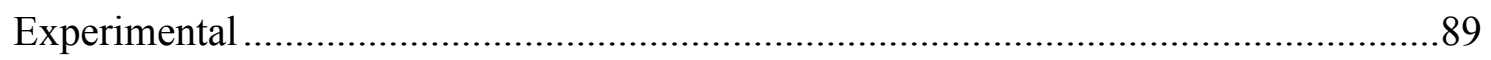

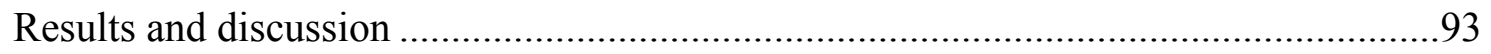

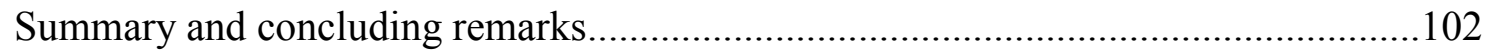

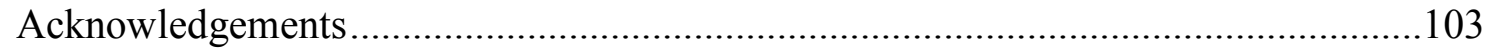

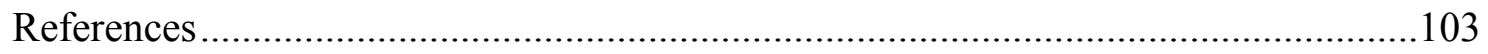

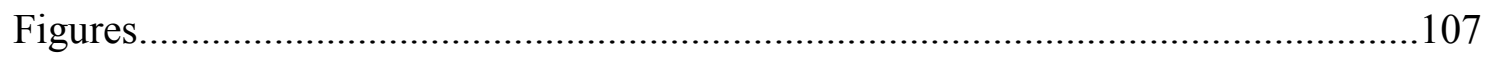

Chapter 6. Data analysis and aging in phosphorescent oxygen-based sensors ......................111

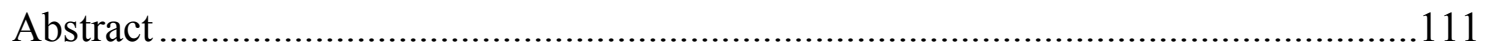

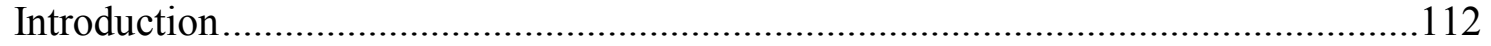

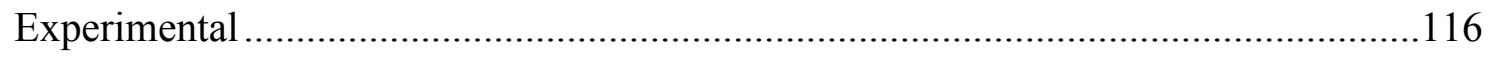

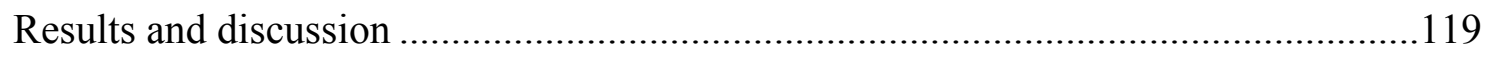

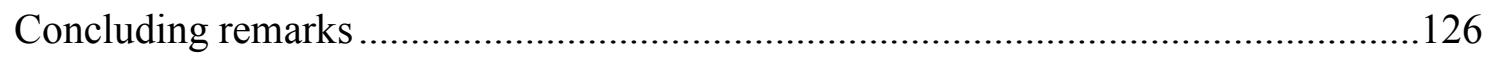

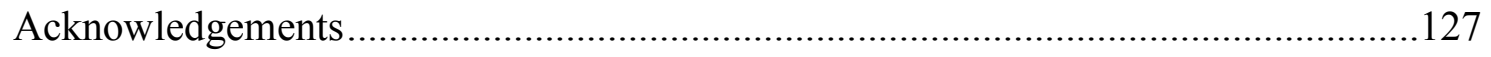

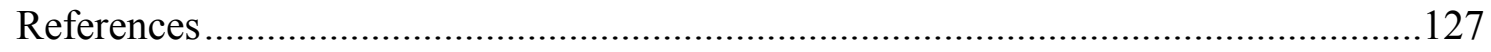

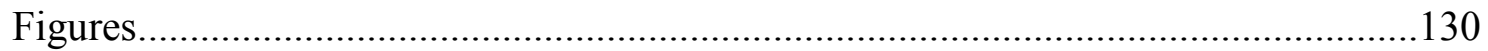


Chapter 7. Polythiophene-fullerene based photodetectors: tuning of spectral response and application in photoluminescence based (bio)chemical sensors...........................137

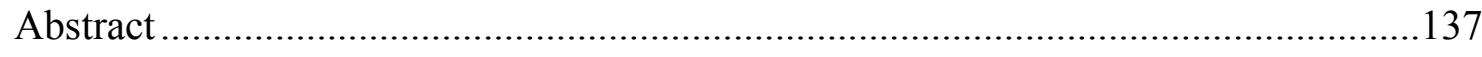

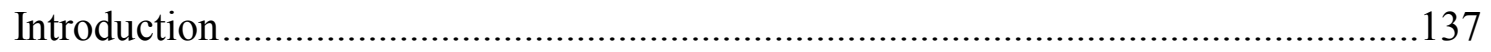

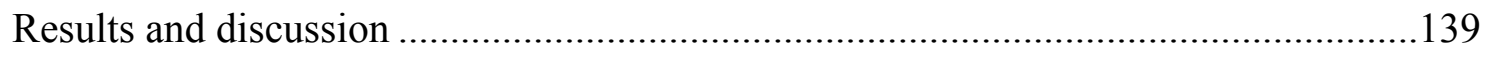

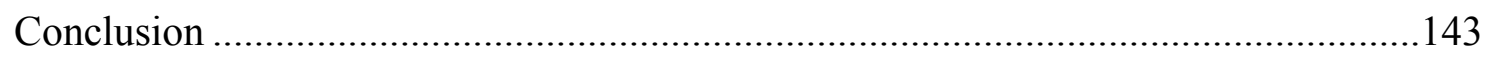

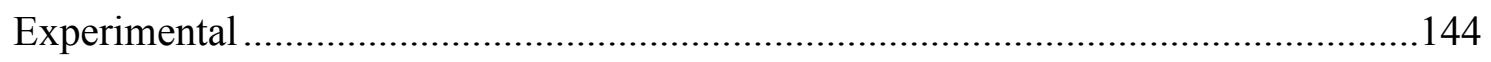

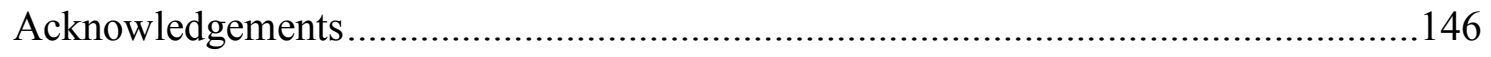

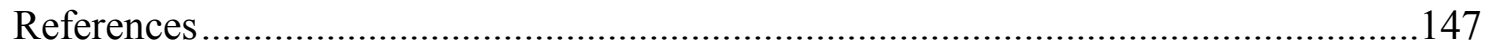

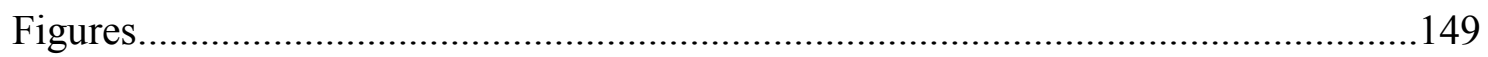

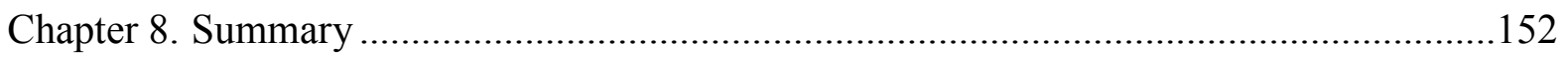

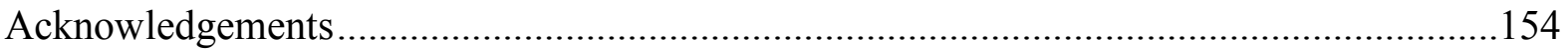




\section{Chapter 1. An overview of OLED basics}

\section{History of organic electroluminescence}

Dark hole injection into an organic crystal was first observed in 1960 by Martin Pope and his group in anthracene. In this seminal discovery, energetic requirements for electron and hole injecting contacts were also described [1]. Three years later, the same group reported the first observation of electroluminescence (EL) from single crystal anthracene and an impurity-doped one under direct current. However, the devices were 10-20 $\mu \mathrm{m}$ thick and only gave off visible emission above $400 \mathrm{~V}$ [2]. Following that, Helfrich and Schneider succeeded in producing double injection recombination EL in anthracene single crystal using hole and electron injecting electrodes. The voltage was significantly reduced to $\sim 60 \mathrm{~V}$ for observable emission. The electron mobility was estimated to be $\sim 0.4 \mathrm{~cm}^{2} /(\mathrm{V} . \mathrm{s})$, based on Child's law. However, the device was 1-5 mm thick, which resulted in a weak current of $10^{-}$

${ }^{10}$ A even at $100 \mathrm{~V}$ [3]. In 1982, Vincentt et al. used vacuum-deposited organic thin film (0.6 $\mu \mathrm{m})$ to achieve EL. The operation voltage was significantly lowered below $100 \mathrm{~V}$. However, the external quantum efficiency (EQE) still remained very low $(\sim 0.05 \%)$ [4].

In the ground-breaking work of Tang et al. in the 1980s, a novel structure was generated with two thin-film organic layers (total thickness $\sim 135 \mathrm{~nm}$ ) independently responsible for hole and electron transport. Higher EQE (1\%), power efficiency $(1.5 \mathrm{~lm} / \mathrm{W})$ and brightness $\left(>1000 \mathrm{Cd} / \mathrm{m}^{2}\right)$ were achieved at a driving voltage below $10 \mathrm{~V}$. Additionally, the device showed rectifier behavior, giving rise to the term OLED (organic light emitting diode) [5]. This discovery stimulated explosive development of this field. 
In parallel to these developments of crystal and amorphous organic-based EL devices, people started to look into the polymers as early as in the mid 1970s, probably motivated by the superior processibility. First reported successful attempt to create polymer LEDs was by UK's national physical lab using a poly(vinylcarbazole) (PVCz) film [6]. In 1990, Friend and co-workers reported highly efficient green-emitting polymer using spin-coated poly( $p$ phenylene vinylene) (PPV) thin film (100nm). The EL was assigned to the radiative decay of singlet excitons formed by injected electrons and holes. The EQE was $0.05 \%$ and the threshold for substantial current injection was around $14 \mathrm{~V}[7]$.

Baldo's work in 1998 broke the $25 \%$ internal efficiency limit [8] by harvesting triplet excitons using the phosphorescent dopant material platinum octaethylporphine (PtOEP). Peak EQE of $4 \%$ was achieved. This set another milestone since Tang's discovery. Later on, Adachi et al. pushed the $\mathrm{EQE}$ to $\sim 22 \%$, which translates to $\sim 100 \%$ internal quantum efficiency, using a phosphorescent dopant in a high band-gap host [9]. Since $\sim 10$ years ago, the third class of OLED materials (dendrimers), has started to draw significant attention, in addition to small molecules and polymers. A typical dendrimer is composed of a core, dendrons, and surface groups. Processing and electronic properties of surface groups and cores can be optimized independently. The number of dendrons provides molecular control over the intermolecular interactions that are crucial for device performance. Moreover, fluorescence and phosphorescence can be easily accessed through the cores. In 2002, using phosphorescent conjugated dendrimers, Lo et al. achieved device performance 5 fold better than polymer and comparable to evaporated molecules at the time [10]. 


\section{OLED applications}

OLEDs are extremely thin $(<0.4 \mu \mathrm{m}$, excluding the substrate $)$ and compatible with flexible substrates. This is uniquely advantageous in terms of device integration. They are easy to fabricate and polymer LEDs are solution processible, which renders the use of roll-toroll manufacturing possible, leading to cost effective mass production. Unlike LCDs, OLEDs are self-luminous without the need for backlight and polarizers. As a result, they are power efficient and have extremely high contrast ratio. Another advantage is their large viewing angle, which can exceed $160^{\circ}$. The emitting surface of OLEDs is normally flat, which causes a Lambertian emission profile. That is, the brightness appears to the same regardless of the viewing angle. Full color gamut is accessible through a variety of organic luminescent materials, which have been developed to cover the whole visible spectrum ranging from near ultra-violet to near infrared. Additionally, the turn-on time for OLEDs is very short and limited by the drift time before electron and hole recombination. The drift time can be

approximately given by $\frac{l^{2}}{\mu \cdot V}$, where $l$ is the length, $\mu$ is the carrier mobility and $V$ is the applied forward bias. Based on the normal conditions: thickness $100 \mathrm{~nm}$, voltage $10 \mathrm{~V}$, mobility $10^{-4}$ to $10^{-5} \mathrm{~cm}^{2} /(\mathrm{V} . \mathrm{s})$, the drift time is estimated to be $\sim 0.1-1 \mu \mathrm{s}$. The radiative decay time is $<100 \mathrm{~ns}$ for fluorescence and $<10 \mu$ s for phosphorescence. Therefore the turn-on and switching time are way below the limit of human sensation.

Due to the aforementioned advantages, OLEDs are promising in two major applications, solid state lighting and displays, which could potentially become competitive compared to existing display technologies. In fact, OLED display products have already presented in the market. For example, Nokia cell phone 6215i, Agilent U1253A handheld 
digital multi-meter, and Nikon Coolpix camera all feature OLED displays. Late in 2009, Sony launched the very first OLED TV Xel-1 into the US market. Amazingly, it is only $3 \mathrm{~mm}$ thick, consumes power of only $45 \mathrm{~W}$, and has a contrast ratio of 1,000,000:1 [11]. Although OLED technology is mainly used in displays, industrial players such as Osram Sylvania, Novaled, GE, and Konica Minolta have been working to produce lighting products. Performance ranging from $\sim 40-64 \mathrm{~lm} / \mathrm{W}$ with a half-life span up to $100,000 \mathrm{hrs}$ at initial brightness of 1000 nits has been announced [12]. In academia, Sun et al. have reported the state-of-art performance of white OLEDs with peak efficiency of $68 \mathrm{~lm} / \mathrm{W}$ by harvesting singlet and triplet excitons as well as wave-guided loss, using low index grid at the organic/ITO interface and microlens at ITO/glass interface [13]. Although progress has been achieved continuously, technological challenges remain to be solved for blue emitting materials, which have high bandgaps and are susceptible to degradation. Complicated control electronics needs to be in place in order to maintain the color balance and purity for display and lighting applications, if RGB colors degrade differently. It is crucial to understand the degradation mechanisms to refine the architecture and materials for reasonable operational lifetime, when compared to other existing technologies.

\section{OLED degradation}

OLED degradation mechanisms can be categorized into extrinsic and intrinsic, depending on whether they are due to the external stimulus or not. Water and $\mathrm{O}_{2}$ have been identified as the major extrinsic sources in the atmosphere to degrade OLEDs. This was implied in the work of Burrows, where simple glass encapsulation in $\mathrm{N}_{2}$ atmosphere increased the OLED lifetime by 2 orders of magnitude [14]. Schaer reported that $\mathrm{H}_{2} \mathrm{O}$ is 1000 
times more destructive than $\mathrm{O}_{2}$ [15]. Experimental evidence was provided by lifetime and microscope measurements with OLEDs exposed to $\mathrm{H}_{2} \mathrm{O}$ and $\mathrm{O}_{2}$ separately, indicating that dark spot formation was 1000 times faster in $\mathrm{H}_{2} \mathrm{O}$ than in $\mathrm{O}_{2}$. The effect of $\mathrm{O}_{2}$ is probably to cause oxidation of metal and organic in the bulk. It was concluded that electrochemical reaction of $\mathrm{H}_{2} \mathrm{O}$ generates $\mathrm{H}_{2}$, which caused delamination of the electrode [15]. To prevent $\mathrm{H}_{2} \mathrm{O}$ and $\mathrm{O}_{2}$ penetration, advanced barrier films (Barix ${ }^{\mathrm{TM}}$ ) were developed using roll-coating techniques. The films are transparent and flexible, made up by evaporated multilayers of polyacrylate and vapor barrier $\mathrm{Al}_{2} \mathrm{O}_{3}[16]$. The moisture permeation rate of the encapsulation layer was determined to be $<10^{-5} \mathrm{~g} / \mathrm{m}^{2} /$ day.

Intrinsic degradation has also been indentified since 1990s, which includes organic and metal diffusion [17], cationic tris(8-hydroxyquinolinato) aluminum $\left(\mathrm{Alq}_{3}\right)$ in $\mathrm{Alq}_{3}$-based OLEDs [18], charge trap and luminescence quencher formation resulting from chemical reactions, and thermal-induced morphology change [19]. Intrinsic degradation could be alleviated by resorting to better materials and device structures, although current understanding is still limited.

\section{Basic structure and operation}

A state-of-art OLED structure typically consists of multiple layers, with each layer responsible for a certain function. As shown in Fig. 1, the OLED starts with $\sim 150 \mathrm{~nm}$ thick ITO, followed by hole injection layer (HIL), hole transport layer (HTL), emission layer (EML), electron transport layer (ETL), electron injection layer (EIL) and $\sim 100 \mathrm{~nm}$ thick metal cathode. The total thickness of the organic layers is $\sim 100 \mathrm{~nm}$. Excluding the encapsulation layer and substrate, the thickness of the active layers is $\sim 350 \mathrm{~nm}$ in total. 


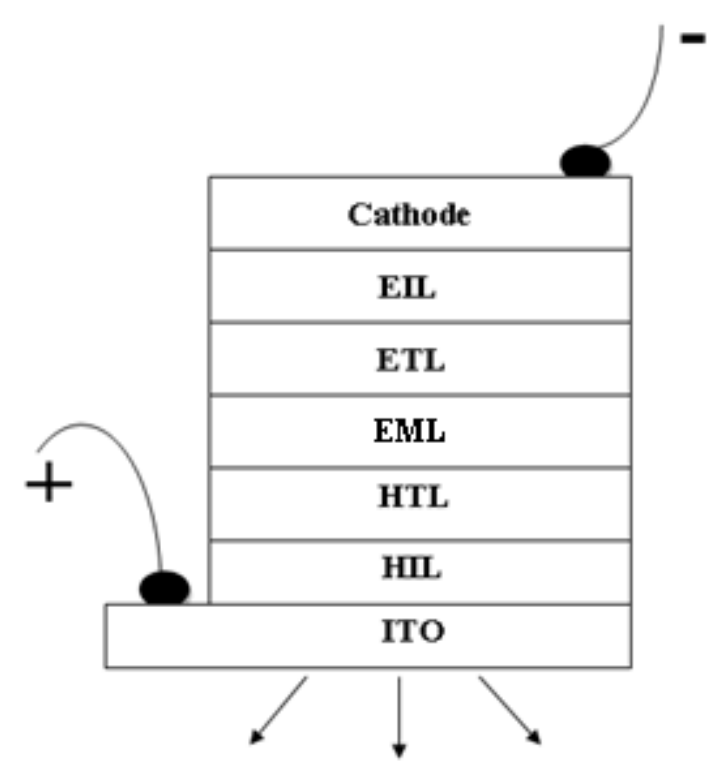

Fig. 1. Multi-layer OLED structure (not to scale)

Figure 2 shows the operation principle of an OLED, when a forward bias is applied, holes and electrons are injected and drifted under the influence of the external field. They meet at the recombination zone, where excitons are formed. Radiative relaxation of the excitons generates photons, part of which exit from the transparent side of OLEDs.

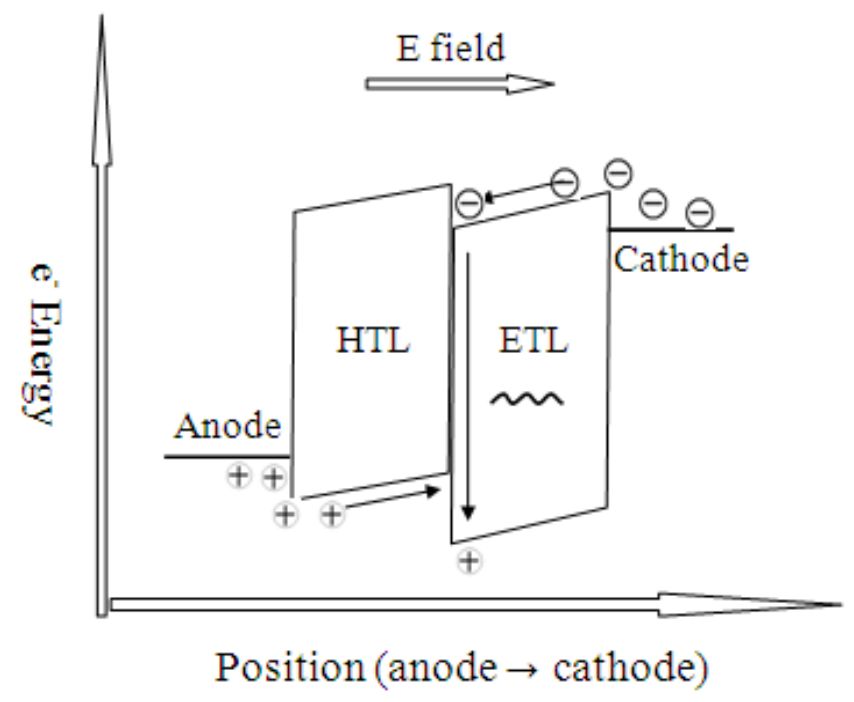

Fig. 2. Operation principle of simple two-layer structure 


\section{OLED physics}

\section{Organic semiconductors}

Organic semiconductors are $\Pi$-conjugated materials, i.e., materials in which single and double bonds or single and triple bonds alternate throughout the molecule or polymer backbone. In bonding via $\mathrm{sp}^{2}$ hybridization, one $\mathrm{s}$ and two $\mathrm{p}$ orbitals of a carbon atom form three hybridized orbitals, one of which can generate a strong $\sigma$ bond with that of a neighboring carbon atom. The un-hybridized $\mathrm{P}_{\mathrm{z}}$ orbital is perpendicular to the plane formed by the hybridized orbitals. Two $\mathrm{P}_{\mathrm{z}}$ orbitals of adjacent two carbon atoms form a weak п bond, where the electrons are delocalized and can move freely in the molecule, which contributes to the semiconducting properties.

Organic semiconductors are usually in the form of amorphous structures in OLEDs. The organic small molecules or polymers interact with one another through Van der Waals forces, which are much weaker than covalent bonding in the inorganic counterparts. Consequently, the carriers are transported by site-to site hopping through the molecules/polymer segments instead of bank-like transport. As a result, the mobility is several orders of magnitude lower. The typical mobility is $\sim 10^{-6}-10^{-3} \mathrm{~cm}^{2} /(\mathrm{V} . \mathrm{s})$ [20], where it is $10^{2}-10^{3} \mathrm{~cm}^{2} /$ (V.s) for inorganic materials. Moreover, the energy structure is described by highest occupied molecular orbital (HOMO) and lowest unoccupied molecular orbital (LUMO), in analogy to valence and conduction bands. Another property that distinguishes between organic and inorganic materials is the dielectric constant $\left(\epsilon_{\mathrm{r}}\right)$, which is $\sim 3$ and $\sim 10$ for organic and inorganic, respectively. Therefore the excitons experience different levels of dielectric screening, which partially determines the exciton binding energy. In inorganic 
materials, the so-called Mott-Wannier excitons have a typical binding energy of $\sim 10 \mathrm{meV}$ [21], while the Frenkel excitons of the organic molecules have a binding energy ranging from $\sim 0.2-1 \mathrm{eV}$.

\section{Current injection and transport}

\subsection{Barrier lowering by image charge}

When the contact between the metal and the organic material is established, there are some electrons transferred from the metal to the organic materials (probably into trap states) [20] via diffusion, provided it is energetically favorable. When the electron is at distance $x$

away from the metal surface, there is an induced positive charge located at $-\mathrm{x}$. Therefore the potential experienced by the electron due to the image charge is

$$
\phi_{\text {image }}=-\mathrm{q}^{2} /(16 \pi \epsilon \mathrm{x}), \epsilon=\epsilon_{\mathrm{o}} \epsilon_{\mathrm{r}}
$$

The effective potential barrier with applied field is given by

$$
\phi_{\mathrm{B}}=\phi_{\mathrm{m}}-\mathrm{q} /\left(\operatorname{Ex}_{\mathrm{m}}\right)-\mathrm{q}^{2} /\left(16 \pi \epsilon \mathrm{x}_{\mathrm{m}}\right)
$$

$\mathrm{x}_{\mathrm{m}}$ is the distance where the sum of the field and image charge term has a maximum, which is

$$
\Delta \phi=-\sqrt{\frac{q^{3} E}{4 \pi \varepsilon}}
$$

The energy diagram is shown in Fig. 3.

Organic semiconductors are intrinsically undoped with low level of thermally generated carriers at room temperature. The actual density of carriers induced by impurities in organic semiconductors is given by [23]:

$$
n_{i}=N_{o} \cdot \exp \left(-\frac{E_{g}}{2 k T}\right)
$$


For a typical value of $E_{g}=2.5 \mathrm{eV}$; and $\mathrm{N}_{0}=10^{21} \mathrm{~cm}^{-3}, \mathrm{n}_{\mathrm{i}}$ is $1 \mathrm{~cm}^{-3}$. Electrons are localized on the molecules and tails of energy states broadened by disorder. Charge carriers are injected from an extended band-like state of the metal electrode into the localized molecular polaronic state in the organic materials. There are two theories modeling the carrier injection behavior, i.e., thermionic emission and field assisted tunneling.

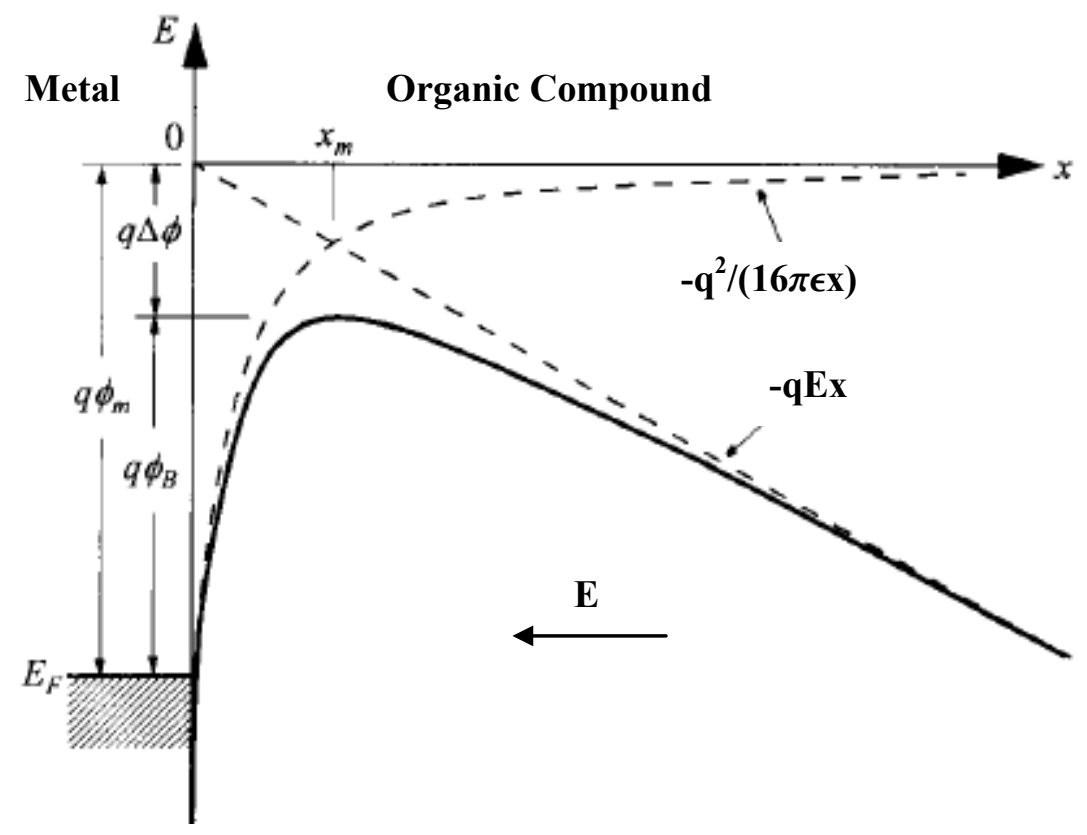

Fig. 3. Barrier lowering by image charge [22]

$\Delta \phi:$ Barrier lowered

$\phi_{\mathrm{m}}$ : Injection barrier (not considering image charge effect)

$\phi_{\mathrm{B}}$ : Injection barrier (considering image charge effect)

\subsection{Thermionic emission}

The essential assumption of this model is that an electron from the metal can be injected, once it acquires a thermal energy sufficient to surpass the potential energy maximum resulting from the superposition of image charge and external field contributions. 
Obviously, it has temperature dependence as shown in the Eq. 1.5, which is also called Richardson-Schottky formula.

$$
J_{t h}=A * T^{2} * \exp \left(\frac{-\emptyset_{b}}{K T}\right)
$$

where $\mathrm{A}$ is Richardson constant, and $\phi_{\mathrm{b}}$ is the barrier height modified by the image charge.

$$
A=\frac{4 \pi q m k^{2}}{h^{3}}
$$

$\mathrm{m}$ is the effective carrier mass. Due to the field-dependent barrier lowering, $J_{\mathrm{th}}$ increases with increasing bias. This represents the greatest current that can flow across the interface when no scattering occurs. However, when both the mobility of the ejected carrier and the applied field are low, the carriers can backflow into the electrode. In this regime, the current is diffusion-controlled. Emtage et al. [24] solved the diffusion-drift equation for injection into a wide bandgap semiconductor and specified the condition for diffusion-limited case, which is $\mu E^{\frac{3}{4}} \ll 5, \mu$ being of the unit of $\mathrm{cm}^{2} / \mathrm{V}$.s and $\mathrm{E}$ in $\mathrm{MV} / \mathrm{cm}$. It was also derived that

(1) In the low field limit $E \ll \frac{4 \pi \varepsilon k^{2} T^{2}}{q^{3}}$

$$
J=N \epsilon \mu E e^{\frac{-q \varphi_{0}}{k T}}
$$

(2) In the high field limit

$$
J=N \mu \sqrt{\frac{k T}{\pi}} \sqrt[4]{4 e E^{3} \epsilon} e \frac{-q \emptyset_{b}}{k T}
$$

$\phi_{\mathrm{o}}$ is the original barrier, and $\phi_{\mathrm{b}}$ is the image charge-modified barrier. This thermal injection process was proved by both Monte Carlo simulations [25] and experiments [26]. 


\subsection{Field assisted tunneling}

At low temperatures and high fields, field-assisted tunneling can be important compared to thermionic emission. Fowler-Nordheim (FN) tunneling model ignores the image charge effects and invokes tunneling of electrons from the metal through a triangular barrier, which can be made thin by applying a high field. It predicts that

$$
\begin{aligned}
& J \propto \frac{E^{2}}{\varnothing} \exp \left(-\frac{b}{E}\right) \\
& b=\frac{8 \pi \sqrt{2 m^{*}} \varphi^{3 / 2}}{3 q h}
\end{aligned}
$$

$\phi$ is the metal-organic barrier. Apparently, FN tunneling current has stronger electrical field dependence than thermionic emission current. Yang et al.[27] have demonstrated the FN tunneling type of unipolar conduction in poly[2-methoxy-5-(2'-ethyl-hexyloxy)-1,4phenylene vinylene] (MEH-PPV)-based OLEDs at high fields ranging from $\sim 0.5$ to $\sim 1.5 \times 10^{6}$ $\mathrm{V} / \mathrm{cm}$. Estimated from this model, barrier height at polyaniline (PANI)/MEH-PPV interface is approximately half that at ITO/MEH-PPV interface, which is consistent with the IV characteristic. However, the FN tunneling theory does not consider the image charge lowering, which amounts to $0.06-0.28 \mathrm{eV}$ at electrical fields between $10^{5}$ and $2 \times 10^{6} \mathrm{~V} / \mathrm{cm}$ in a dielectric with $\epsilon_{\mathrm{r}}=3.5$. As Bassler et al. mentioned [28], the barrier lowering may be comparable to the barrier height. As a result, the conduction is no longer injection limited, which renders FN tunneling model invalid. 


\subsection{Space charge limited current (SCLC)}

In modern OLEDs [20], standard EL operation requires injection $>3 \mathrm{~mA} / \mathrm{cm}^{2}$. However, the carrier mobility is low, being $10^{-6}-10^{-4} \mathrm{~cm}^{2} /(\mathrm{V} . \mathrm{s})$ for electrons and $10^{-5}-10^{-3}$ $\mathrm{cm}^{2} /$ (V.s) for holes. Such strong injection into low mobility materials inevitably leads to charge accumulation in organic materials. Mott-Curney relation, also known as Child's law, for trap-free unipolar conduction follows:

$$
J=\frac{9 \epsilon \mu V^{2}}{8 L^{3}}
$$

where $\mu$ is the mobility, $\epsilon$ is the dielectric permittivity, $\mathrm{V}$ is the applied voltage, and $L$ is the thickness of the sample. In the presence of shallow traps, the mobility can be thought of as "reduced effective mobility" with a pre-factor determined by the concentrations of the free and trapped charges.

$$
\begin{aligned}
& J=\frac{9 \epsilon(\theta \mu) V^{2}}{8 L^{3}} \\
& \theta=\frac{P_{f}}{P_{t}}
\end{aligned}
$$

$P_{f}$ is the free carrier concentration and $P_{t}$ is the trapped carrier concentration $[29,30]$.

It should be noted that SCLC dominates at high current, where traps are filled and the electrode/organic contact is Ohmic or quasi-Ohmic. In the work of Mori et al. [31], SCLC, however, was not observed in multilayer small molecular OLEDs. It was not only attributed to failure of the Ohmic contact presumption at the electrode/organic interface, but also to the energy barriers between the organic layers. On the contrary, the SCLC model is easily 
accepted in much simpler structures, such as polymer LEDs [32] and single layer hole-only small molecular devices [33].

\subsection{Trap charge limited current (TCLC)}

Disorder, electron-phonon interaction, low bandwidth, impurities, dopants, and degradation products can all be sources to trap the carriers. The distribution of trap energies is assumed to be Gaussian or exponential. Before the trap-free SCLC limit is reached, the bulk conduction is limited by trap filling of the carriers. Burrows et al. [34] modeled the IV with an exponential trap distribution below the LUMO states, with a characteristic energy $E_{t}$ $=0.15 \mathrm{eV}$ below the $\mathrm{LUMO}$ of $\mathrm{Alq}_{3}$. The IV relation is given by

$$
J \propto \frac{V^{\frac{T_{t}}{T}+1}}{d^{\frac{2 T_{t}}{T}+1}}
$$

where $T_{t}=E_{t} / k$ is the characteristic temperature of the trap distribution. If $T_{t} \gg T$, the measurement temperature, we can assume that the electrons are full below the electron quasiFermi level and empty above it. From temperature measurements, $T_{\mathrm{t}}$ was determined to be $1780 \mathrm{~K}$, thus most of the traps are indeed deep traps as required by the model. The trap density was found to be $3 \times 10^{18} \mathrm{~cm}^{-3}$, seven orders of magnitude larger than the estimated thermally generated carrier density. Calculations showed that the nature of the traps could be structural relaxation of $\mathrm{Alq}_{3}$ anion, which forms polaronic excited states.

Over the full range of applied bias, the IV characteristic may go through Ohmic, injection limited, shallow-trap SCLC / TCLC, and trap free SCLC. At very low fields, the

Ohmic conduction $J=q p_{0} \mu \frac{V}{d}$ due to the thermally generated carriers, may be important. 
However, the thermally generated carriers have very low concentration $<10^{11} \mathrm{~cm}^{2} /$ V.s. As a result, the Ohmic current is comparable to the noise level of the probe and not easily accessible [34]. As the field increases, the thermionic emission with a supralinear dependence on electrical field takes over. This could be replaced by FN tunneling current with further increase in the field. If the injection contact is Ohmic or the injection barrier is modified to be very small by image charge potential induced by high field, then bulk limited current becomes important, e.g., shallow-trap SCLC. As the injection level cranks up even further, quasi-Fermi level moves toward the LUMO for electrons and HOMO for holes, traps are being filled by TCLC. Following that, trap free SCLC dominates. In a work by Adachi et al. [31], an extremely high current density of $125 \mathrm{kA} / \mathrm{cm}^{2}$ was achieved through a CuPc film by using a small pixel size of $7.9 \mu \mathrm{m}^{2}$. FN tunneling current, shallow trap SCLC, and trap free SCLC were sequentially observed with increasing voltage.

\subsection{Mobility}

The temperature and electrical field dependence of the mobility was ignored in the aforementioned models. However, in many cases, variable mobility can be adopted to explain the IV characteristics very well $[35,36]$. Schein et al. [37] reported for the first time the temperature and electric field dependence of the mobility in a molecularly-doped polymer using hole-drift measurements. The empirical functional form was given by

$$
\mu=\mu_{0} \exp \left[-\left(\frac{T_{1}}{T}\right)^{2}\right] \exp \left\{\sqrt{E}\left[\left(\frac{\beta}{T}\right)-\gamma\right]\right\}
$$

Or simply, it can be written as $\mu(E, T)=\mu(0, T) \exp (y \sqrt{E})$, which has the field dependence of Poole-Frenkel type. In this model, the electrons are generally trapped in 
localized states. Occasionally, random thermal fluctuation will give the carrier enough energy to escape the localized state and move to the conduction band. Thus electrons can move through the crystal for a brief amount of time before relaxing into another localized state. Carrier transport in organic materials can be considered in a picture similar to this. Moreover, the potential of the trap states with a trap charge can be distorted asymmetrically under applied bias in a way similar to barrier lowering by image charge force. Poole-Frenkel type of mobility has been successfully demonstrated in IV characteristics of OLEDs [35, 36].

\subsection{Recombination}

If the oppositely charged carriers are injected statistically independent of each other, then the random process can be described by Langevin formalism [38]. In order to be bonded, the electron-hole pair requires that Coulombic attraction be larger than the thermal energy as shown in the following:

$$
\frac{q^{2}}{4 \pi \epsilon r}>k T
$$

Onsager radius $r_{c}$ is the maximum separation for electron-hole capture: $r_{c}=\frac{q^{2}}{4 \pi \epsilon k T}$. It is typically $\sim 17 \mathrm{~nm}$ at room temperature. Electron and hole pair within this radius will recombine and form excitons. The capture process is bimolecular and can be viewed as one carrier being static and another one drifting toward it with effective mobility $\mu_{m}=\mu_{e}+\mu_{h}$. It can be shown that the recombination efficiency is $R=\gamma p n$, where $\gamma=e \mu_{m} / \epsilon$ and $\mathrm{p}$ and $\mathrm{n}$ are concentrations of holes and electrons, respectively. 
As implied by the Langevin model, the capture process is spin-independent. In other words, the probability of formation of triplet excitons from charge transfer state in triplet configuration is the same as that of singlet excitons from charge transfer state in singlet configuration. Consequently, the ratio of triplet to singlet is 3 to 1 , which limits the maximum internal efficiency of fluorescent OLEDs to $25 \%$. However, there are suggestions that in polymers, the formation cross section for singlets and triplets are not equal $[39,40]$. As a result, the singlet ratio is larger than $1 / 4$. However, this issue is still being heavily debated $[41,42]$.

\section{Electronic processes in organic materials}

\subsection{Frank-Condon principle}

Organic small molecules or polymer typically comprise at least hundreds of atoms, making analytic quantum analysis too complicated. Therefore the analysis of molecular transitions relies on the Born-Oppenheimer (B-O) approximation, which allows the wavefunction of a molecule to be broken into its nuclear and electronic components. In other words, electrons can be approximated to respond instantaneously to the movement of the nuclei and the electronic transitions take place with the nuclei configuration stationary because of their large mass difference. The electronic transition, such as photon absorption

and emission occur in $10^{-15} \mathrm{~s}$ and molecule relaxation happens in $10^{-13} \mathrm{~s}$, which further validates this approximation [43].

B-O approximation results in Frank-Condon principle in optical transitions. Electrons are photo excited to the vibrational levels of higher electronic states vertically with 
the nuclear configuration unchanged. Then the electrons relax to the lowest manifold of excited state with a new nuclear equilibrium position before reaching the ground state. Upon emission of a photon vertically, the spectrum is red-shifted, which is termed as the FrankCondon shift. It may be as large as $1 \mathrm{eV}$ in some molecules, making them transparent to their own emission.

The molecular electronic transition is proportional to the electronic dipole moment between the initial and final states, predicted by Fermi-Golden rule. There are certain conditions to allow the transition to happen, i.e., symmetry of the transition states or spin, as shown next.

\subsection{Spin conservation rule}

In the ground state of most stable organic molecules, the HOMO is filled by two electrons with opposite spins based on the Pauli Exclusion Principle. Electrons at the HOMO level are most likely to participate in electron transfer or optical transitions. The remaining electrons occupy tightly filled orbitals. Therefore the system can be described by a twoelectron problem, which gives either total spin 0 or 1 . According to degeneracy, $\mathrm{S}=0$ is called singlet and $\mathrm{S}=1$ triplet with spin wavefunctions as follows:

$$
\begin{aligned}
& \varphi_{s=0}=\frac{1}{\sqrt{2}}[\uparrow(1) \downarrow(2)-\downarrow(1) \uparrow(2)] \\
& \varphi_{s=1, s_{Z}=0}=\frac{1}{\sqrt{2}}[\uparrow(1) \downarrow(2)+\downarrow(1) \uparrow(2)] \\
& \varphi_{s=1, s_{Z}=1}=\uparrow(1) \uparrow(2) \\
& \varphi_{s=1, s_{Z}=-1}=\downarrow(1) \downarrow(2)
\end{aligned}
$$


The total wavefunction of the two-electron system has to be anti-symmetric under electron interchange. As a result, the spatial wavefunction is symmetrical for singlet and antisymmetric for triplet.

The dipole moment operator is $-\mathbf{e} \mathbf{R}_{\mathbf{1}}-\mathrm{e} \mathbf{R}_{\mathbf{2}}$, which is symmetric under electron exchange. Therefore, if the spatial wavefunction of the initial and final states have different symmetry, the transition dipole moment

$$
\mu=-e<\psi_{f}\left(\boldsymbol{R}_{\mathbf{1}}, \boldsymbol{R}_{\mathbf{2}}\right)\left|\boldsymbol{R}_{\mathbf{1}}+\boldsymbol{R}_{\mathbf{2}}\right| \psi_{i}\left(\boldsymbol{R}_{\mathbf{1}}, \boldsymbol{R}_{\mathbf{2}}\right)>
$$

will be zero under electron exchange, otherwise it changes sign [44]. However, the dipole moment does not depend on the labeling of the electrons. We can conclude that spin wavefunctions of the initial and final states must have the same symmetry as the spatial components. Thus, only singlet-singlet and triplet-triplet optical transitions are allowed.

\subsection{Spin-orbit coupling [44]}

Singlets and triplets can be mixed by spin-orbital coupling, making the triplet singlet transition possible. A heavy metal atom in the center of an organic molecule, i.e., platinum octaethylporphrine (PtOEP), promotes strong spin-orbit coupling. The interaction energy of the magnetic dipole moment due to spin and magnetic field generated by orbiting the center is given by $-\boldsymbol{\mu} . \boldsymbol{B}$. The magnetic field $\boldsymbol{B}$ is related to the angular momentum $\boldsymbol{l}$. The additional term of the Hamiltonian due to this interaction can be expressed as $H_{s-o} \propto \frac{Z}{r^{3}} \boldsymbol{l}$. s. Considering this as the hydrogen model with a modified core, the expectation value of $\mathrm{r}^{-3}$ is proportional to $Z^{3}$. Therefore, the total interaction is proportional to $Z^{4}$. It can be shown that the singlet can be coupled to triplet as 


$$
<1,0\left|H_{s-o}\right| 0,0>=\frac{\hbar}{2}\left(\xi l_{1 z}-\xi l_{1 z}\right)
$$

where $H_{s-o}=\xi \boldsymbol{l} . \boldsymbol{s}$.

Optical transition from a triplet excited state to a singlet ground state becomes allowed via the mixed singlet character of the triplets induced by spin-orbit coupling. This resulted in phosphorescence, which has a relatively long decay time, i.e., $100 \mu$ s for PtOEP in a polymer matrix in the absence of external quenchers $[45,46]$. Spin-orbit coupling also encourages the intersystem crossing (ISO) from excited singlet states to triplet states due to the same reason. Fluorescence is differently due to radiative relaxation from excited singlet state to ground singlet states.

\subsection{Energy transfer}

Molecular excited state (exciton) can transfer its energy over to another molecule through radiative energy transfer, Förster energy transfer, or Dexter Energy transfer. The latter two are commonly used in OLED technologies to improve the efficiency.

In radiative energy transfer, the emission given off by the donor molecule is followed by the absorption of the photon by the acceptor molecule. Obviously, it can occur if only the emission spectrum of the donor overlaps the absorption spectrum of the acceptor.

Energy transfer can occur via dipole-dipole interaction. This is the Forster energy transfer, which takes place within $10 \mathrm{~nm}$ at a time scale of $10^{-9} \mathrm{~s}$ [44]. 


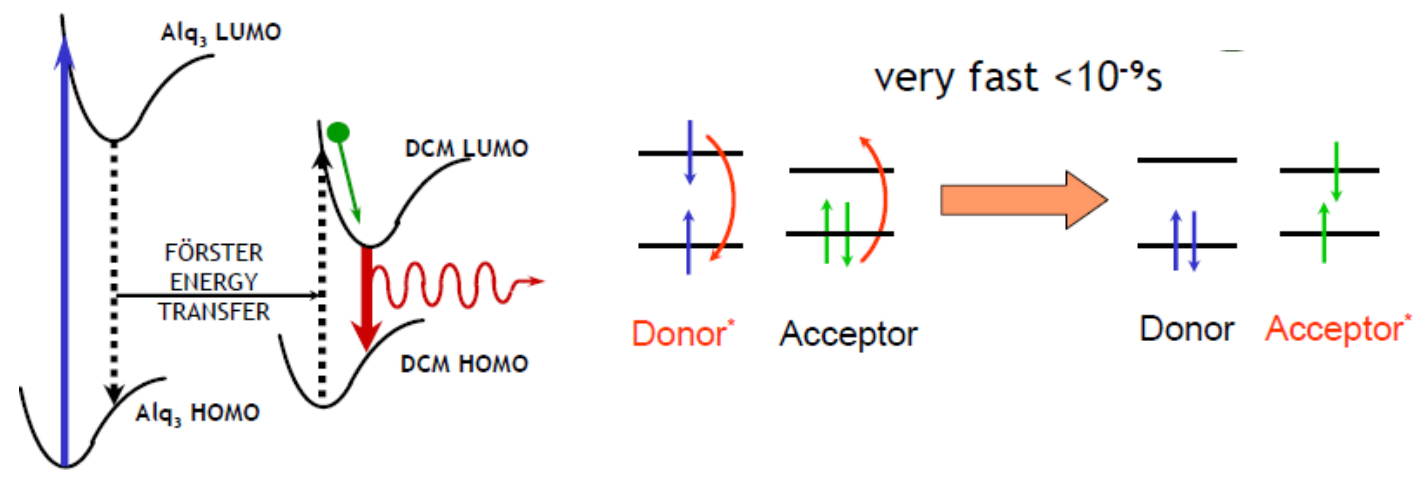

Fig. 4. Forester energy transfer [47]

Following the Fermi-Golden rule, one can derive the transition probability for Forster Energy transfer:

$$
K_{\text {Förster }}(R)=\frac{3 \hbar^{4} c^{4}}{4 \pi \epsilon_{r}^{2} R^{6}} \frac{\alpha_{a}}{N_{a}} \frac{1}{\tau_{d}} \int \frac{F_{D}(E) F_{A}(E)}{E^{4}} d E
$$

where $\alpha_{a}=\int \alpha d E$ is the total absorption coefficient of the acceptor. $N_{a}$ is density of the acceptor molecules. $\tau_{d}$ is the lifetime of the donor. $F_{D}(E)$ is the normalized donor emission spectrum and $F_{A}(E)$ is the normalized acceptor absorption spectrum. R is the donor-acceptor separation.

$$
\begin{aligned}
& K_{\text {Förster }}(R)=\left(\frac{1}{\tau_{d}}\right)\left(\frac{R_{0}}{R}\right)^{6} \\
& R_{0}^{6}=\frac{3 \hbar^{4} c^{4}}{4 \pi \epsilon_{r}^{2}} \frac{\alpha_{a}}{N_{a}} \int F_{D}(E) F_{A}(E) / E^{4} d E
\end{aligned}
$$

$R_{0}$ can be interpreted as the distance, where the energy transfer rate equals the total deactivation rates by all the other means. Transfer efficiency can be expressed as:

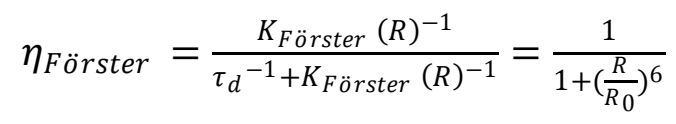


The spin selection rule $\Delta S=0$ applies for both donor and acceptor. Thus the allowed transitions are:

$$
\begin{aligned}
& { }^{1} D^{*}+{ }^{1} A \rightarrow{ }^{1} D+{ }^{1} A^{*} \\
& { }^{1} D^{*}+{ }^{3} A \rightarrow{ }^{1} D+{ }^{3} A^{*}
\end{aligned}
$$

Spin-orbit coupling allows for optical transitions with $\Delta S \neq 0$ and the actual radiative lifetime of the triplet states are long; therefore the energy transfer process $\left({ }^{3} D^{*} \rightarrow{ }^{1} D\right)$ may still be important relative to radiative process of the triplets of the donors. This is the Förster triplet-singlet transition [43]:

$$
{ }^{3} D^{*}+{ }^{1} A \rightarrow{ }^{1} D+{ }^{1} A^{*}
$$

Baldo et al. has demonstrated this in phosphor sensitized fluorescence and achieved tripled efficiency by converting all triplets into radiative singlets [48].

Another type of energy transfer is via electron exchange, which occurs at very short range $(\sim 1 \mathrm{~nm})$. The is the Dexter energy transfer, which only requires the total spin conservation of the donor-acceptor system as a whole, thus triplet-triplet and triplet-singlet energy transfers

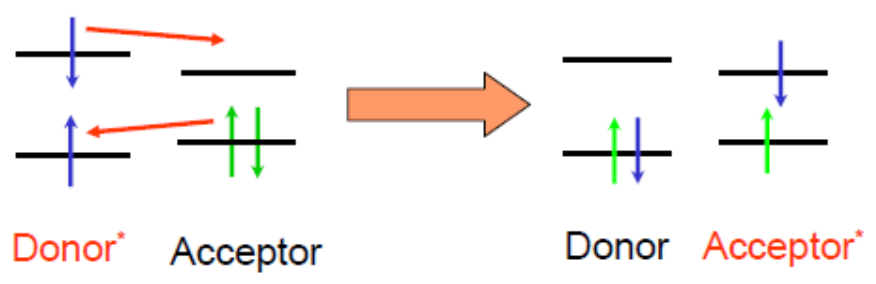

Fig. 5. Dexter energy transfer [47]

are allowed. The first one forms the basis of $\mathrm{O}_{2}$ sensing, using a phosphorescence dye as a probe, which shows reduced photoluminescence (PL) intensity and shortened decay time in the presence of $\mathrm{O}_{2}$ due to quenching. Although, in principle, Dexter energy transfer between 
singlet and singlet is allowed, the Förster type occurs much faster and covers a longer range. Therefore, this type of Dexter energy transfer is usually negligible. Electron exchange is a short range process, critically dependent on the distance $R$ between donor and acceptor. The transfer rate is given by

$$
K_{\text {Dexter }} \propto J \exp \left(-\frac{2 R}{L}\right)
$$

where $\mathrm{J}$ the spectral overlap integral and $\mathrm{L}$ is a characteristic distance [43].

\section{OLED efficiency \& enhancement}

OLED design and research effort fundamentally and critically relies on the detailed understanding of the factors that affect the OLED efficiency. In this section, the commonly used measures of efficiency, the contributing factors, and how to determine the values from experimental observables are discussed. Following that, more detail is provided to

demonstrate the enhancement of OLED performance based on improvement of each individual factor.

\subsection{OLED efficiencies}

\subsubsection{OLED efficiency basics}

External quantum efficiency (EQE) is defined as the ratio of the number of photons extracted toward the front to that of electrons flowing through the external driving circuit. It can be broken into four factors:

$$
\eta_{E Q E}=\xi \gamma r_{s t} \eta_{P L}
$$


Due to the difference of the refractive indices, as photons go through different layers such as organic, ITO and glass before exiting the device, there is a significant fraction of photons trapped, waveguided or re-absorbed within the device, not being able to contribute to the forward light emission. The out-coupling efficiency $\xi$ represents the fraction for the extracted photons out of the total internally generated ones. Together with the generation of an electron, there is a hole at the opposite electrode under applied bias. However, because of different interfacial barriers and trap states, electrons and holes are not injected equally. The radiation originates from the electron-hole pair recombination. We use the balance factor $\gamma$ to address the concern over the imbalanced pairing. As mentioned in the previous sections, in fluorescent OLEDs, only singlet excitons can be harvested according to the spin conservation rule. Singlet excitons, however, typically account for only $25 \%$ of the total excitons based on spin-statistics. $r_{s t}$ is the ratio of the radiative exictons to all excitions generated. There are non-radiative decay channels competing with the radiative one. Thus, only a fraction of the radiative excitons, which is represented by the photoluminescence (PL) quantum yield $\eta_{P L}$, can eventually produce photons.

Another measure, power efficiency $\left(\eta_{p w}\right)$ in the unit of $1 \mathrm{~m} / \mathrm{W}$, is also widely used to give an idea about how much perceived optical power (luminous power) can be generated per unit input electrical power. This provides a direct energy basis for comparison between different lighting technologies. Note that human eyes are not equally sensitive to different wavelengths with a distribution peaking at $555 \mathrm{~nm}$. 1 watt of photons at this wavelength is perceived as 683 lumens by the human eyes. The distribution $\phi(\lambda)$ is expressed by the photopic response curve as shown in Fig. 6. 


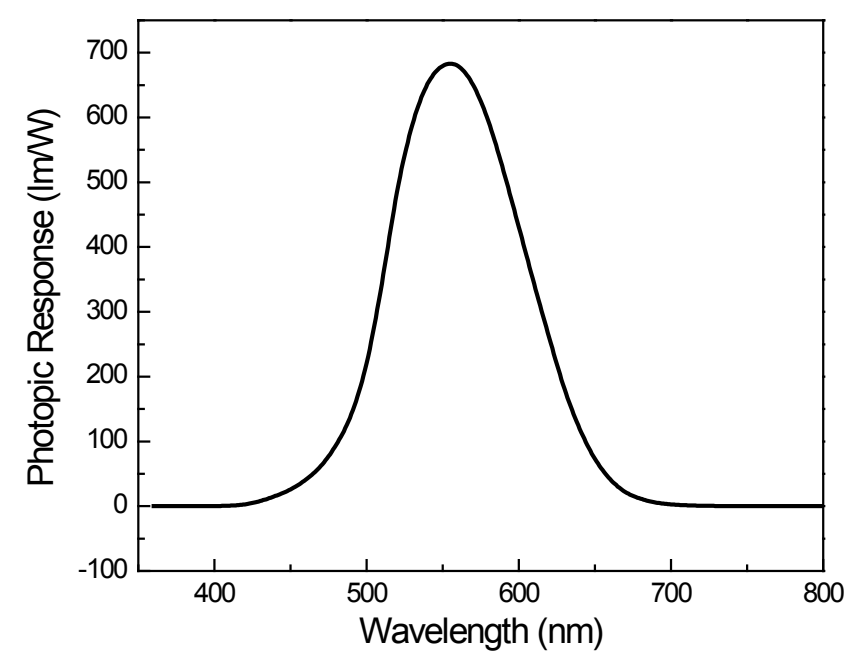

Fig. 6. Photopic response of human eyes

The normalized photopic response curve is the luminosity function $\psi(\lambda)$.

Luminance, in unit of $\mathrm{Cd} / \mathrm{m}^{2}$, corresponds to the brightness in general terms. Usually, this is a directly measured quantity, which can be converted to luminous current efficiency $(\mathrm{Cd} / \mathrm{A})$, given the current density going through the device. Candela $(\mathrm{Cd})$ is a SI base unit of luminous intensity, which is a measure of wavelength-weighted power emitted by a light source at a certain direction per unit solid angle.

\subsection{Efficiency calculation}

As mentioned above, the luminous current efficiency can be obtained from experimental observables. Due to the usually flat exiting surface of OLED, the emission profile is approximately Lambertian. It means that the luminous power ( $\mathrm{lm}$ ) follows a cosine law with the viewing angle $\theta$ subtended with respect to the normal direction of the emitting surface or the brightness is the same regardless of the viewing angle, assuming that the 
microcavity effect is weak and the spectrum remains the same at all viewing angles. The relation between luminous power and luminance is given by

$$
L=\frac{d^{2} F}{d A d \Omega \cos \theta}
$$

$\mathrm{F}$ is the luminous power, $\mathrm{L}$ is the luminance and $\mathrm{A}$ is the area of emitting source. The total luminous flux contained in the forward half viewing hemisphere is

$$
F=\int L \cos \theta d A d \Omega=A L \pi
$$

where $L$ is independent of $\theta$. The total input power obtained by the measured current and voltage combined with the perceived optical power estimated according to Lambertian profile yields the luminous power efficiency in $1 \mathrm{~m} / \mathrm{W}$.

For single wavelength emission, the external quantum efficiency is related to the power efficiency by:

$$
\left(\eta_{E Q E}=\frac{\# \text { photons out }}{\# \text { electrons in }}\right) \cdot \frac{e_{V_{\lambda}} / \Delta t}{e V / \Delta t} \cdot \phi(\lambda)=\eta_{p w}
$$

$V_{\lambda}$ is the potential difference of the initial and final states of the optical transition that gives off the photon. $V$ is the applied voltage and $\phi(\lambda)$ is the photopic response. Assuming the total photon number emitted per unit time is $\mathrm{N}_{\mathrm{p}}$, the normalized spectrum is $\frac{E L(\lambda)}{\int E L(\lambda) d \lambda}$, and the total luminous power is $L A \pi=\frac{\int E L(\lambda) h v_{\lambda} \phi(\lambda) d \lambda}{\int E L(\lambda) d \lambda} N_{p}$. It can be shown that the external quantum efficiency is related to the measured luminance and spectrum as follows:

$$
\eta_{E Q E}=\frac{N_{p}}{I / e}=\frac{\pi e}{h c} \frac{L}{J} \frac{\int E L(\lambda) d \lambda}{\int \frac{E L(\lambda) \phi(\lambda)}{\lambda} d \lambda}
$$




\subsection{Efficiency enhancement}

\subsubsection{Exciton harvesting}

Spin-statistics predicts that $25 \%$ of the electron-hole pairs formed after injection are singlets and $75 \%$ are triplets. Although this has been debated, especially for polymers [39, 40], there is considerable experimental evidence supporting this claim [41, 42]. Most of the organic materials for OLEDs are fluorescent, allowing only radiative decay of singlet excited state to singlet ground state. Thus, $75 \%$ of the excitons are lost to non-radiative recombination. The maximum internal quantum efficiency, therefore, is limited to $25 \%$.

In light of this challenge, Baldo et al. [8], introduced phosphorescent materials to harvest the triplets. Strong spin-orbit coupling, owing to the heavy metal core such as in PtOEP, mixes the triplet states with singlet character, making radiative transition of the triplets possible. It also encourages intersystem crossing from the excited singlet to triplet state, due to the same reason. In principle, $100 \%$ of the excitons are emissive and the total the internal quantum efficiency can be as high as 100\%. Forrest's group reported a high peak external quantum efficiency of $4 \%$ and internal quantum efficiency of $23 \%$, using PtOEP in a host material. Furthermore, Adachi et al. [9] even demonstrated nearly $100 \%$ internal quantum efficiency, assuming that the out-coupling factor is $\sim 20 \%$ and the peak luminous power efficiency of $60 \mathrm{~lm} / \mathrm{W}$. This high performance was additionally attributed to direct exciton formation at the guest phosphor molecules within a high bandgap host.

Very few organic materials have been found to exhibit efficient room-temperature phosphorescence and triplet-triplet annihilation at high excitation density also affects the 
emission efficiency. Consequently, fluorescent materials are more suited to many electroluminescent applications. Baldo et al. [48] managed to channel the triplets into the singlet states of the fluorescent dopants, by introducing a phosphor sensitizer, which allows triplet-singlet energy transfer. More than tripled efficiency was achieved as compared with the case without the sensitizer.

Spin-statistics implies that the charge transfer states, which are the precursors of the excitons, have the same cross section for singlet and triplet exciton formation. However, calculations based on density functional theory exhibits a lower energy level of singlet charge transfer state than triplet charge transfer state. Segal et al. [49] used a phosphor layer to affect only the charge transfer states by mixing the spins. Therefore, the triplet charge transfer states can be transferred to singlet charge transfer states allowed by energetic ordering and spin-orbit coupling. As a result, they tripled the singlet fraction as well as the efficiency of the red fluorophore 4-(Dicyanomethylene)-2-methyl-6-(julolidin-4-yl-vinyl)4H-pyran $\left(\mathrm{DCM}_{2}\right)$-based OLED. This enhanced fluorescence was termed as “extrafluorescence".

\subsubsection{Interface engineering}

Ideally, an Ohmic contact is preferred at the organic/electrode interface for easy carrier injection. Because of the availability of organic and electrode materials, an energy barrier exists at electrode/organic interfaces, which results in high operating voltages and low power efficiencies. 
Hung et al. [50] interposed a thin layer of thermally evaporated LiF between the ETL and cathode to enhance electron injection. Atomic Force Microscopy (AFM) results showed a good coverage of LiF on the ETL and analysis of stoichiometric composition indicated the absence of Li metal, which could also enhance electron injection. The enhanced injection was then attributed to band bending-induced barrier lowering as suggested by the photoelectron emission study, although current understanding indicates that dipole layer formation shifts the vacuum level to lower the injection barrier. Besides $\mathrm{LiF}, \mathrm{MgO}$ and $\mathrm{Al}_{2} \mathrm{O}_{3}$ have also been shown to work [50,51].

At the anode/organic interface, inserting copper phthalocyanine $(\mathrm{CuPc})$ was found to improve the injection current, efficiency and stability of the devices $[52,53]$. The HOMO of CuPc was found to be suited between that of HTL such as NPB and the work function of ITO. It is widely accepted that the energy ladder structure promotes the carrier injection. Additionally, $\mathrm{MoO}_{3}, \mathrm{WO}_{3}$, surface treatment by UV-Ozone, etc. [54] have all been demonstrated to enhance hole injection, owing to reduced energy barrier caused by i.e., dipole layer-induced vacuum level shift and an intermediate energy level provided by the inserted materials.

\subsubsection{Conductivity doping}

Organic semiconductors have very low concentrations of intrinsic thermally generated carrier $\left(<10^{11} \mathrm{~cm}^{-3}\right)$ at room temperature $[23,34]$. Thus, the resistance is high and a much higher operating voltage than the minimum potential $\left(V_{\lambda}\right)$ is required to generate photons. Conductivity doping, however, can alleviate this problem. Pfeiffer et al. [55] reported a PIN structure, where the HTL 4,4',4"-tris(3-methylphenylphenylamino) 
triphenylamine (m-TDADA) was doped with strong electron acceptor tetrafluorotetracyanoquinodimethane (F4-TCNQ) to generate holes and the ETL was Li-doped 4,7diphenyl-1,10-phenanthroline (BPhen). Li contributes a free electron concentration of $\sim 10^{18}$ $\mathrm{cm}^{-3}[56]$. The electron blocking layer tris(1-phenyl- $\kappa C^{1}$-pyrazolato- $\left.\kappa N^{2}\right)$ iridium $\left(\operatorname{Ir}(\mathrm{ppz})_{3}\right)$ and exciton blocking layer pure Bphen were deposited on the HTL and ETL sides of the intrinsic emission layer, respectively. Both fluorescent and phosphorescent PIN structures have been demonstrated with an operating voltage $2.55-2.65 \mathrm{~V}$ for $100 \mathrm{Cd} / \mathrm{m}^{2}$ i.e., close to the minimum driving voltage $V_{\lambda}[55,57]$. Another advantage is that doped devices can be made much thicker without appreciably increasing the voltage drop. This considerably improves the device yield when large displays are mass produced by reducing the parasitic shorts between electrodes encountered in undoped thinner devices.

\subsubsection{Outcoupling enhancement}

OLEDs are planar multi-layer structures and photons generated inside experience layers with different refractive indexes before exiting the transparent side, which is usually glass. In small molecular OLEDs, the distribution of emitting dipoles is usually isotropic. For this type of dipoles not subject to optical interference, optical simulation shows that the outcoupling efficiency $\xi$ can be obtained by integrating the intensity over the surface-escape cone and taking the ratio to the hemispherical emission. Then

$$
\xi=\int_{0}^{\theta_{c}} \sin \theta d \theta=1-\cos \theta_{c}=1-\sqrt{\left(1-\frac{1}{n^{2}}\right)} \approx \frac{1}{2 n^{2}}(\text { for large } \mathrm{n})
$$


where $\mathrm{n}$ is the refractive index of the organic layers. Kim's analysis [58] also predicts that $\xi$ varies as $0.75 n^{-2}$ for isotropic emitter and as $1.2 n^{-2}$ for the in-plane case with the emitting dipoles optimally located for maximum rate of surface emission.

Based on this analysis, we can conclude only $\sim 20 \%$ of the total internally generated photons can escape into the front viewing hemisphere [9]. Concomitantly, $\sim 40-60 \%[59,60]$ are waveguided and confined within ITO and organic layers based on analysis of finitedifferent time-domain, mode expansion, and ray optics. The loss at the glass/air interface due to total internal reflection is possibly $\sim 20-40 \%$. This imposes challenges and generates opportunities to further enhance the efficiency.

Sun et al. [61] reported $\sim 50 \%$ increase in the front emission by using microlens fabricated by imprint lithography on the glass side compared to the conventional device. Optimal size of the microlens, contact angle, and refractive index of the microlens were also determined from FDTD (finite-difference time-domain) and Monte Carlo ray tracing simulations. It was pointed that when the size of the microlens gets close to the wavelength, results from FDTD and Monte Carlo ray tracing diverge. It indicates that ray optics may not be applicable any more for that case. One more step forward, Sun [62] patterned a low index grid (LIG) using photolithography on PECVD-deposited $\mathrm{SiO}_{2}$ layer, which was between the ITO and organic layers. LIG combined with microlens generated total enhancement factors of 2.3 compared to conventional device and $\sim 1.68$ for microlens only, which implies a further $\sim 40 \%$ increase in out-coupling efficiency by LIG on top of the microlens. Simulation also predicts that enhancement can be further increased to 3.4 using LIG with microlens of 
even lower index $(\leq 1.3)$. This effort leads to the state-of-art performance for white emitting OLEDs with peak external quantum efficiency of $34 \%$ and power efficiency of $68 \mathrm{~lm} / \mathrm{W}$.

\subsubsection{Materials of high PL quantum yield}

The intrinsic PL quantum yield of OLED materials is usually less than 1 and depends on the material. Organic laser dyes such as Coumarin, Rhodamin, etc. with nearly $100 \%$ PL quantum yield can be doped into OLEDs. On the contrary, the most widely used archetypal fluorescent small molecules i.e., tris(8-hydroxyquinolinato) ( $\left.\mathrm{Alq}_{3}\right)$ only have PL quantum yield of $\sim 32 \%$ [63]. Consequentially, for example, the 2,3,6,7-Tetrahydro-1,1,7,7,tetramethyl-1H,5H,11H-10-(2-benzothiazolyl) quinolizino [9,9a,1gh] coumarin (C545T) doped green OLEDs are 3-4 more efficient than those based on $\mathrm{Alq}_{3}$. Also, nearly 10\% external quantum efficiency has been achieved based on the highly efficient fluorescent dopant C545T [64].

\section{OLED fabrication}

Small molecular OLEDs are normally prepared by thermal evaporation of the thin film layers in a high vacuum chamber $\left(<10^{-6}\right.$ mbar $)$ housed in $\mathrm{Ar} / \mathrm{N}_{2}$ filled glovebox. The layers' thickness can be accurately controlled by a thickness monitor placed in the vicinity of the substrate. On the contrary, polymers are not suited for thermal evaporation, because they are large in mass and tend to decompose at high temperatures. Wet processing techniques are therefore applied, such as spin-coating, screen printing, and inkjet printing. For these cases, the thickness cannot be as easily controlled. However, wet processing presents a promising outlook for large volume manufacturing, which requires high processibility and low cost. 
Technologies have been also developed for vapor-based high yield deposition such as organic vapor phase deposition and organic vapor jet printing for potential scalable processes.

\section{References}

[1] H. Kallmann, M. Pope, Nature, 186 (1960) 31.

[2] M. Pope, H.P. Kallmann, P. Magnante, J. Chem. Phys., 38 (1963) 2042.

[3] W. Helfrich, W.G. Schneider, Phys. Rev. Lett., 14(7) (1965) 229

[4] P.S. Vincett, W.A. Barlow, R.A. Hann, G.G. Roberts, Thin Solid Films, 94(2) (1982) 171.

[5] C.W. Tang, S.A. VanSklyke, Appl. Phys. Lett. 51(2) (1987) 913.

[6] R.H. Patridge, Polymer 24(6) (1983) 748; R.H.Patridge, Polymer, 24(6) (1983) 755.

[7] J.H. Burroughes, D.D.C. Bradley, A.R. Brown, R.N. Marks, K.Mackay, R.H. Friend, P.L. Burns, A.B. Holmes, Nature, 347 (1990) 539.

[8] M.A. Baldo, D.F. O’Brien, Y. You, A. Shoustikov, S. Sibley, M.E. Thompson, S.R. Forrest, Nature, 395 (1998) 151.

[9] C. Adachi, M.A. Baldo, M.E. Thompson, S.R. Forrest, J. Appl. Phys., 90(10) (2001) 5048.

[10] S. Lo, N Male, J. Markham, S. Magennis, P. Burn, O. Salata, I. Samuel, Adv. Mater., 14 (13-14) (2002) 975.

[11] www.sonystyle.com, www.nokia.com, www.agilent.com, www.nikon.com

[12] www.konicaminolta.com, www.novaled.com, www.osram.com

[13] Y. Sun, S.R. Forrest, Nature Photonics, 2 (2008) 483.

[14] P.E. Burrows, V. Bulovic, S.R. Forrest, L.S. Sapochak, D.M. McCarty, M.E. Thompson, Appl. Phys. Lett., 65(23) (1994) 2922.

[15] M. Schaer, F. Nuesch, D. Berner, W. Leo, L. Zuppiroli, Adv. Funct. Mater., 11(2) (2001) 116.

[16] P.E. Burrows, G.L. Graff, M.E. Gross, P.M. Martin, M.K. Shi, M. Hall, E. Mast, C. Bonham, W. Bennett, M.B. Sullivan, Display, 22 (2001) 65. 
[17] S. T. Lee, Z.Q. Gao, L.S. Hung, Appl. Phys. Lett., 75(10) (1999) 1404.

[18] H. Aziz, Z.D. Popvic, N. Hu, A. Hor, G. Xu, Science, 283 (1999) 1900.

[19] F. So, Organic Electronics: Materials, Processing, Devices and Applications, CRC press (2010).

[20] J. Shinar, Organic Light-Emitting Devices, AIP press (2002).

[21] C. Kittel, Introduction to Solid State Physics, $6^{\text {th }}$ ed., John Wiley \& Sons (1986).

[22] S.M. Sze, Physics of Semiconductor Devices, $3^{\text {rd }}$ ed., John Wiley \& Sons (2007).

[23] W. Brutting, Physics of organic semiconductors, Wiley-VCH (2005).

[24] P.R. Emtage, J.J. O’Dwyer, Phys. Rev. Lett., 16(9) (1966) 356.

[25] Y. Gartstein, E.M. Conwell, Chem. Phys. Lett., 255 (1996) 93.

[26] M. Abkowitz, H. Mize, K. Facci, Appl. Phys. Lett., 66 (1995) 1288.

[27] Y. Yang, A.J. Heeger, Appl. Phys. Lett. 64(10) (1994) 1245.

[28] V. I. Arkhipov, E.V. Emelianova, Y. H. Tak, H. Bassler, J. Appl. Phys., 84(2) (1998) 848.

[29] T. Matsushima, H. Sasabe, C. Adachi, Appl. Phys. Lett., 88 (2006) 033508.

[30] M.A. Lampert, Phys. Rev., 103(6) (1956) 1648.

[31] T. Mori, T. Ogawa, D. Cho, T. Mizutani , Appl. Sur. Sci., 212-213 (2003) 458.

[32] A. J. Campbell, D. Bradley, H. Antoniadis, M. Inbasekaran, W. Wu, E. Woo, Appl. Phys. Lett., 76(13) (2000) 1734.

[33] C. Giebeler, H. Antoniadis, D. Bradley, Y. Shirota, Appl. Phys. Lett., 72(19) (1998) 2448.

[34] P.E. Burrows, Z. Shen, V. Bulovic, D.M. McCarty, S.R. Forrest, J.A. Cronin, M.E. Thompson, J. Appl. Phys., 79 (10) (1996) 7991.

[35] W. Brutting, S. Berleb, A.G. Muck1, Synthetic Metals, 122 (2001) 99.

[36] T. Chu, O. Song, Appl. Phys. Lett., 90 (2007) 203512. 
[37] L.B. Schein, A. Rosenberg, S.L. Rice, J. Appl. Phys., 60(12) (1986) 4287.

[38] M. Pope, C.E. Swenberg, Electronic Processes in Organic Cyrstals, Oxford University Press, Oxford (1982).

[39] M. Wohlgennant, K. Tandon, S. Mazumdar, S. Ramasesha, Z.V. Vardeney, Nature, 409 (2001) 494.

[40] Y. Cao, I. Parker, G. Yu, A. J. Heeger, Nature, 397 (1999) 414.

[41] M.A. Baldo, D.F. O’Brien, M.E. Thompson, S.R Forrest, Phys. Rev. B., 60 (14) (1999) 422.

[42] M. Segal, M.A. Baldo, R. J. Holmes, S.R. Forrest, Z.G. Soos, Phys. Rev. B., 68 (2003) 075211.

[43] L. Gang, PhD dissertation (2003).

[44] M. A. Baldo, PhD dissertation (2001).

[45] D.B. Papkovski, Sens. Actuators, B 29, 213 (1995).

[46] R. Shinar, Z. Zhou, B. Choudhury, J. Shinar, Ana. Chim. Acta, 568 (2006) 190.

[47] MIT lecture on organic electronics.

[48] M.A. Baldo, M.E. Thompson, S.R. Forrest, Nature, 403 (2000) 750.

[49] M. Segal, M. Singh, K. Rivoire, S. Difley, T.V. Voorhis, M.A. Baldo, Nature Materials, 6 (2007) 374.

[50] L.S. Hung, C.W. Tang, M. G. Mason, Appl. Phys. Lett., 70 (2) (1997) 152.

[51] H. Tang, F. Li, J. Shinar, Appl. Phys. Lett., 71 (18) (1997) 2560.

[52] S.M. Tadayyon, H.M. Grandin, K. Griffiths, P.R. Norton, H. Aziz, Z.D. Popvic, Organic Electronics, 5 (2004) 157.

[53] S.A. Van Slyke, C.H. Chen, C.W. Tang, Appl. Phys. Lett., 69(15) (1996) 2160.

[54] H. You, Y. Dai, Z. Zhang, D. Ma, J. Appl. Phys., 101 (2007) 026105; J. Li, M. Yahiro, K. Ishida, H. Yamada, K. Matsushige, Synthetic Metals, 151 (2005) 141; S. Y. Kim, J. Lee, J. Appl. Phys., 95(5) (2004) 2560.

[55] M. Pfeiffer, S. R. Forrest, K. Leo, M. E. Thompson, Adv. Mater., 14(22) (2002) 1633. 
[56] G. Parthasarathy, C. Shen, A. Kahn, S.R. Forrest, J. Appl. Phys., 89 (2001) 4986.

[57] J. Huang, M. Pfeiffer, A. Werner, J. Blochwitz, K. Leo, S. Liu, Appl. Phys. Lett., 80(1) (2002) 139.

[58] J. Kim, P. Ho, N. Greenham, R. H. Friend, J. Appl. Phys., 88(2) (2000) 1073.

[59] A. Chutinan, K. ishihara, T. Asano, M. Fujita, S. Noda, Organic Electronics, 6 (2005) 3.

[60] G. Gu, P.E. Burrows, S. Venkatesh, S.R. Forrest, M.E. Thompson, Opt. Lett., 22 (1997) 396.

[61] Y. Sun, S.R. Forrest, J. Appl. Phys., 100 (2006) 073106.

[62] Y. Sun, S. R. Forrest, Nature Photonics, 2 (2008) 483.

[63] D.Z. Garbuzov, V. Bulovic, P.E. Burrows, S.R. Forrest, Chem. Phys. Lett., 249 (1996) 433.

[64] K. Okumoto, H. Kanno, Y. Hamaa, H. Takahashi, K. Shibata, Appl. Phys. Lett., 89 (2006) 063504. 


\section{Chapter 2. General introduction to OLED-based structurally integrated}

\section{optical sensors}

\section{Background}

In addition to solid state lighting and display applications, OLEDs are uniquely adaptable as excitation sources in photoluminescence (PL)-based sensor technologies, owing to their high brightness, uniquely simple integrability, submicron thickness for active layers, flexibility of design for novel applications, miniaturizability and processibility. Unlike traditional light source i.e., lamps, lasers, and inorganic LEDs, OLEDs and sensing elements can be fabricated on a common substrate and photo-detectors can be placed in close proximity without the need for complex optical components for signal control, such as filters, lens, polarizers, collimators, fibers, etc. High brightness $\left(5 \times 10^{6} \mathrm{Cd} / \mathrm{m}^{2}\right)$ [1] has been demonstrated in the course of continued technological advancement over the past 20 years. This is particularly important for operation of optical sensors, in which OLEDs are utilized to provide the excitation light, although the typical operational excitation intensity is far below the peak value. Moreover, the wide availability of OLEDs emitting across the full color gamut suites the needs for different applications requiring varied wavelengths.

Since the seminal work on such an integrated sensor for $\mathrm{O}_{2}$ monitoring about 10 years ago [2], Shinars' group have continuously driven the development of this area, which also generated research interest across the world [3-7]. Potentially, OLED-based structurally integrated sensors offer low cost, fast response, durability, small size and field deployability for efficient multianalyte parallel monitoring of chemical and biological analytes, including those with physiological and industrial significance such as glucose, lactate, cholesterol, 
ethanol and $\mathrm{O}_{2}$. More detail in basics and achievements are elaborated in the following sections.

\section{Sensor basics}

\section{Sensing methods and targets}

Current OLED-based optical sensors can be categorized into two groups, depending upon whether the detected signal is the PL of the sensing element or OLED's waveguided/reflected light. In the latter case, the OLED signal is coupled into the photodetector through waveguiding or reflection, with the light intensity sensitive to the refractive index change of the sensing region in the presence of an analyte, the distance of an object from the OLEDs, or the pressure $[3,7]$.

In OLED-based sensors that monitor the PL, the sensor element usually contains a luminescent dye, whose PL is subject to quenching or enhancement, depending on the analyte type and level and on chemical reactions in the analyte-containing sample; alternatively, the PL of a luminescent reaction product is monitored. As an example of the latter, in the hydrazine sensor, the reaction product of hydrazine and (anthracene 2,3-

dicarboxaldehyde) ADA produces luminescence peaking at $\sim 550 \mathrm{~nm}$, when excited by blue OLEDs. As the PL intensity is monitored in this case, optical filters are used to remove the EL background, allowing passage of only the PL, which is strongly dependent on hydrazine's concentration. Analytes can also be detected by means of their PL quenching e.g., via triplettriplet Dexter energy transfer for $\mathrm{O}_{2}$ sensing using $\mathrm{O}_{2}$-sensitive phosphorescent dyes, or via fluorescence resonance energy transfer for i.e., anthrax lethal factor sensing $[8,9]$. 
$\mathrm{O}_{2}$-based sensing enables the monitoring of other chemical and biological analytes such as glucose, lactate, cholesterol, and alcohol. $\mathrm{O}_{2}$ concentration is altered by the oxidation reaction of the analytes in the presence of their corresponding specific oxidases. The specificity of the detection allows for parallel monitoring of these analytes in a single mixed sample.

Unlike most molecules, the ground state of $\mathrm{O}_{2}$ is a triplet with two unpaired electrons occupying two different orbitals. Therefore $\mathrm{O}_{2}$ is an efficient quencher of triplet excited states. Promoted by strong spin-orbit coupling, dyes such as PtOEP produce $>50 \%$ triplet excitons by photo-excitation and $\sim 90 \%$ of those are able to generate phosphorescence, which enables effective $\mathrm{O}_{2}$ detection based on its PL quenching. From this point forward, the discussion focuses on OLED-based sensors monitoring PL that is subject to quenching by $\mathrm{O}_{2}$.

\section{Sensor components and structures}

The structurally integrated OLED-based sensor is typically composed of three basic components: the OLED excitation source, the sensor film that is fabricated by embedding $\mathrm{O}_{2-}$ sensitive dye in a polymer matrix, and the photodetector (PD). These three components can be arranged in either front detection or back detection geometry, as shown in Fig. 1. In the

front detection geometry, the OLEDs and photodetector are placed on two different sides of the sensor film, while in the back detection geometry, they are on the same side of the sensor film. The back detection geometry typically results in a more compact device and enables easier sample handling. 

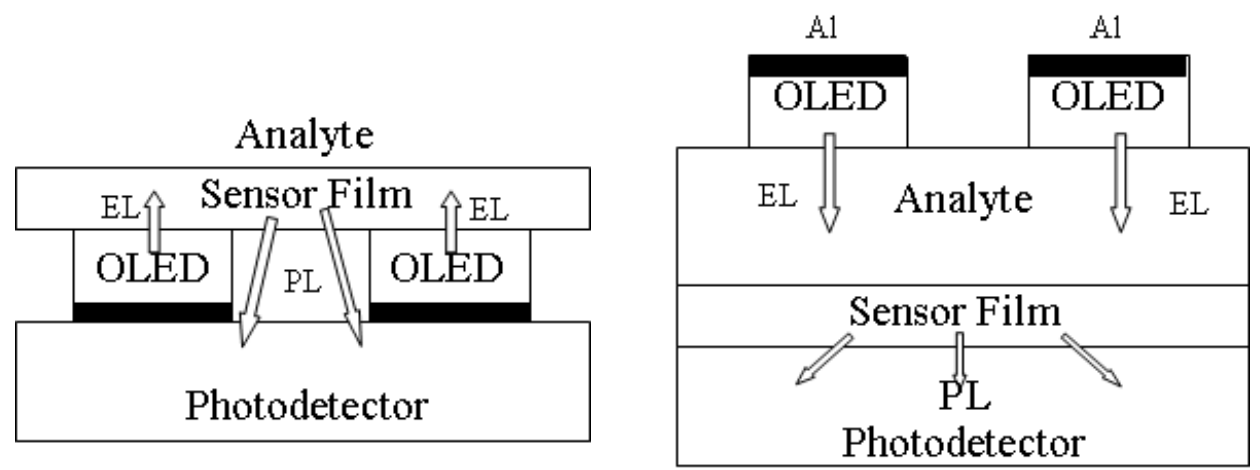

Fig. 1. Back detection (left) and front detection (right) geometries (not to scale)

\section{Calibration and operation}

\subsection{Stern-Volmer relation}

$\mathrm{O}_{2}$ quenches the PL of luminophores by collisions in a dynamic quenching process. Collisional quenching is ideally described by the Stern-Volmer equation [10]:

$$
\frac{I_{0}}{I}=\frac{\tau_{0}}{\tau}=1+K_{s v}\left[O_{2}\right]=1+k_{q} \tau_{0}\left[O_{2}\right]
$$

where $I$ and $\tau$ are the PL intensity and decay time, respectively, in the presence of the quencher, $I_{0}$ and $\tau_{0}$ are the unquenched values, $K_{s v}$ is the Stern-Volmer constant, and $k_{q}$ is bimolecular quenching rate constant.

As shown in the Jablonski diagram in Fig. 2, $\mathrm{O}_{2}$ provides an additional channel to deactivate the triplet excited states. The Stern-Volmer relation can be derived by considering the luminescent intensity observed in the absence and presence of the quencher. The observed intensity of the the PL of the luminophore is proportional to the concentration of the excited states [I*]. A steady state is established under continuous excitation, thus $\frac{d\left[I^{*}\right]}{d t}=0$. In the absence and presence of the quencher, the rate equations are given as follows: 


$$
\begin{aligned}
& \frac{d\left[I^{*}\right]}{d t}=g(t)-\left(k_{p}+k_{n r}\right)\left[I^{*}\right]_{0}=0 \\
& \frac{d\left[I^{*}\right]}{d t}=g(t)-\left(k_{p}+k_{n r}+k_{q}\left[O_{2}\right]\right)\left[I^{*}\right]=0
\end{aligned}
$$

where $\mathrm{g}(\mathrm{t})$ is the excitation function, $k_{\mathrm{p}}$ is the radiative decay rate, and and $k_{\mathrm{nr}}$ is sum of all the other non-radiative decay rates in the absence of $\mathrm{O}_{2}$ Combining these two equations above yields:

$$
\frac{\left[I^{*}\right]_{0}}{\left[I^{*}\right]}=\frac{I_{0}}{I}=\frac{k_{p}+k_{n r}+k_{q}\left[O_{2}\right]}{k_{p}+k_{n r}}=1+\frac{k_{q}}{k_{p}+k_{n r}}\left[O_{2}\right]=1+k_{q} \tau_{0}\left[O_{2}\right]=\frac{\tau_{0}}{\tau}
$$

$\tau_{0}=\frac{1}{k_{p}+k_{n r}}$ and $\tau=\frac{1}{k_{p}+k_{n r}+k_{q}\left[O_{2}\right]}$ (assuming the $\mathrm{T}_{1} \rightarrow \mathrm{S}_{1}$ intersystem crossing is negligible due to energetic ordering)

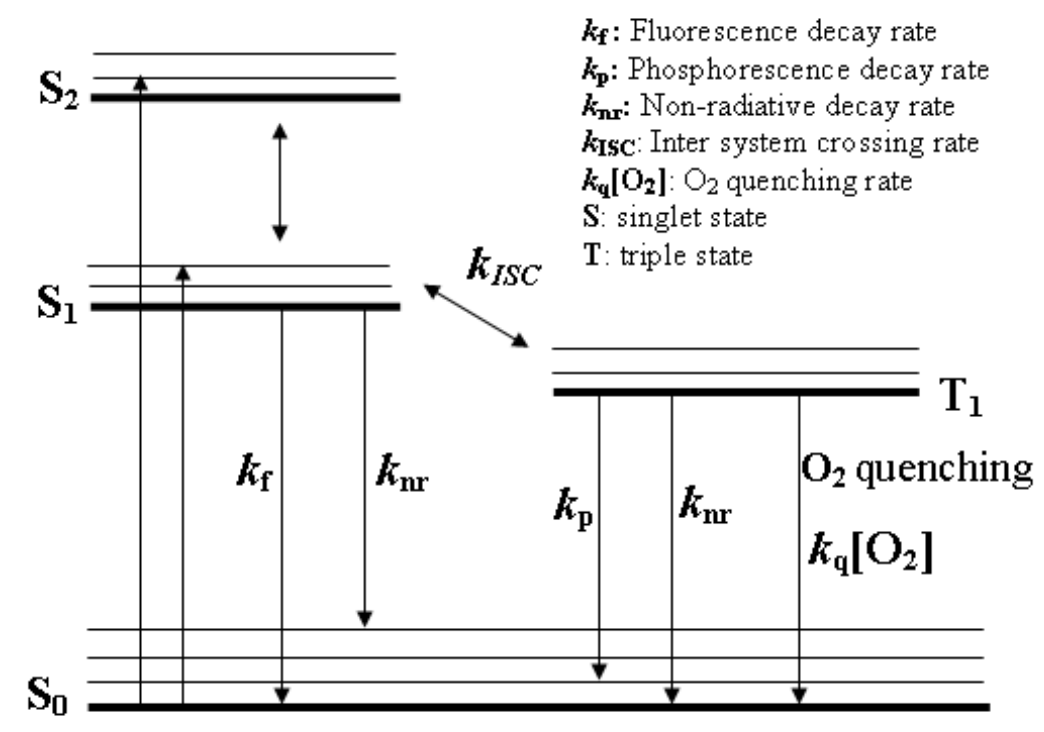

Fig. 2. Jablonski diagram with $\mathrm{O}_{2}$ quenching 


\subsection{Modified Stern-Volmer relation}

If the sensor film is exposed to a buffered analyte solution i.e., glucose and glucose oxidase in a hermetically sealed cell without any air above the solution and $\mathrm{O}_{2}$ replenishing, the calibration curve is given by the modified Stern-Volmer relation [11]:

$$
\frac{I_{0}}{I}=\frac{\tau_{0}}{\tau}=1+K_{s v}\left([D . O .]_{\text {initial }}-[\text { analyte }]_{\text {initial }}\right)
$$

The PL signal is collected after the reaction is complete and correlated with the residual [DO] (concentration of dissolved $\mathrm{O}_{2}$ ), which is determined by the initial analyte concentration $[\text { analyte }]_{\text {initial }}$. The equation above holds if the [analyte $]_{\text {initial }}$ is lower than the initial [DO] in the buffered solution, which is $\sim 0.26 \mathrm{mM}$ at room temperature. If [analyte] $]_{\text {initial }}$ is larger than

$0.26 \mathrm{mM}$, the PL signal remains unchanged after the [DO] depleted even with increasing $[\text { analyte }]_{\text {initial. }}$ The analytes' oxidation reactions, similar to that shown belown for glucose, proceed stoichiometrically to completion.

$$
\text { Glucose }+\mathrm{O}_{2} \stackrel{\mathrm{GOx}}{\longrightarrow} \mathrm{H}_{2} \mathrm{O}_{2}+\text { gluconic acid }
$$

\subsection{Operation modes}

As mentioned, both the PL decay time $\tau$ and steady state intensity $I$ can be used to represent the analyte concentration, but $\tau$ measurement is preferred, where the PL is monitored following an OLED pulse. Pulsing reduces the duty cycle and therefore OLED degradation [12]. Another advantage of pulsing is the higher brightness that can be achieved within the short pulse (usually $100 \mu$ s for PtOEP) for better signal to noise without 
overloading the OLED. In addition, pulsed excitation shortens the light exposure of the sensor film, resulting in reduced photo-bleaching. Furthermore, the decay time measurement is not susceptible to minor OLED degradation and dye leaching, thus eliminating the need for frequent sensor calibration. $\mathrm{O}_{2}$-sensitive dyes have a large range of lifetimes, with $\sim 3-100 \mu \mathrm{s}$ for PtOEP and $\sim 5-1000 \mu$ s for PdOEP [9], corresponding to $100 \% \mathrm{O}_{2}$ and $0 \% \mathrm{O}_{2}$, respectively.

A somewhat different monitoring approach was used when the oxidase, in addition to $\mathrm{O}_{2}$-sensitive dye, was embedded in a solid matrix, rather than in solution. This approach was successfully demonstrated in sensors with the enzyme immobilized in a sol-gel film and to $\mathrm{ZnO}$ nanoparticles $[13,14]$. In this approach, the sensor film is covered by analyte solution, which is exposed to the ambient, leading to constant replenishing of $\mathrm{O}_{2}$ from the atmosphere into the solution. This diffusion rate, however, is relatively slow compared to the $\mathrm{O}_{2}$ consumption caused by the reaction with the analyte and oxidase, particularly at the initial stage of the reaction, and depending on the enzyme level. The initial reaction rate is increased monotonically with increasing analyte concentration in agreement with MichaliesMenten kinetics.

\section{Development of OLED-based structurally integrated sensors}

The first OLED-based structurally integrated sensor developed by Shinars' group was for $\mathrm{O}_{2}$ using a thin-film sensing element. Solution-based sensors for single analytes were also evaluated for e.g., hydrazine and anthrax lethal factor. High sensitivities (defined as the ratio between the $I_{0}\left(\tau_{0}\right)$ and $I(\tau)$ at $\left.100 \% \mathrm{O}_{2}\right)$ were achieved, i.e., $\sim 10$ for DO and $\sim 30$ for gas phase $\mathrm{O}_{2}$ [9], using a sensor film made of PtOEP embedded in polystyrene (PS). 
New possibilities were next explored for monitoring blood serum constituents such as glucose, lactate, cholesterol, and alcohol, with the oxidase dissolved in solution or immobilized in a solid matrix. Obviously, in solution-based sensing, the oxidase has to be replaced after each measurement, resulting in material waste. One approach to immobilize the oxidase is to use sol-gel films. Following hydrolysis and polycondensation of the precursor, the sol-gel process forms an integrated network containing the enzyme within small pores that prevent enzyme leaching. Bhaskar et al. [13] demonstrated a glucose sensor using this technique. $\mathrm{ZnO}$ nanoparticles were also investigated by Cai et al. [14] for enzyme immobilization, which was achieved by physical adsorption and Coulombic attraction. The isoelectric point is $\sim 9.5$ for $\mathrm{ZnO}$ and $\sim 4.5$ for the oxidase. When $\mathrm{ZnO}$ and the oxidase are dissolved in a buffer solution with a $\mathrm{pH}$ value between 4.5 and 9.5 , the $\mathrm{ZnO}$ and oxidase carry opposite charges, which results in electrostatic attraction. As a result, the oxidase is immobilized onto the $\mathrm{ZnO}$ surface. One reason for enzyme immobilization is to enable repeated use in order to lower the material cost and enhance the durability of the sensor. Furthermore, sensor handling is simplified with one less step of introducing the oxidase. Additional details regarding this topic are given in chapter 4.

To further improve the structural integrability of the OLED sensing paltform, the bulky photomultiplier tube (PMT) was replaced by thin film photodetectors (based on amorphous or nanocrystalline silicon) or by commercial silicon photodiode. Ghosh et al. introduced Ge into amorphous silicon to lower the bandgap in order to shift the response curve of the thin film photodetector toward the PL maximum of the sensor film [15]. The intensity mode of operation was successfully demonstrated on this platform utilizing lock-in 
detection and electromagnetic shielding of PD. However, Ge introduced trap states which slowed down photo-generated carriers and lead to recombination loss. Thus, the overall EQE was reduced. The best thin-film Si photodetectors were based on nanocrystalline Si, but their response time was too long $(\sim 200 \mu \mathrm{s})$, which prevented PL decay time measurements.

This challenge was partially solved by using poly(3-hexylthiophene) (P3HT): phenylC61-butyric acid methyl ester (PCBM)-based photodetectors, which were successfully employed together with the OLEDs for glucose and gas phase $\mathrm{O}_{2}$ sensing. The response time of the PD was estimated to be $\sim 10 \mu \mathrm{s}$, (based on transient photocurrent measurement following a light pulse), which limits the maximum concentration $\mathrm{O}_{2}$ that can be measured, since the PL decay time of PtOEP in response to $100 \%$ gas phase $\mathrm{O}_{2}$ is $3-5 \mu \mathrm{s}$. This, however, does not affect the performance of the glucose sensor and other similar DO-based sensors, as room-temperature $[\mathrm{DO}]_{\text {initial }}$ corresponds to decay time of 25-30 $\mu \mathrm{s}$, which is longer than the PD response time. This work is discussed in more detail in Chapter 7. The combination of polymer PD and OLED provides the opportunity for an all-organic sensor platform, which could potentially be fabricated by low-cost and large volume processes. Sensors based on OPDs + OLEDs have also been shown for monitoring $\mathrm{pH}$ value, refractive index change and distance $[3,5]$. They can even be fabricated on the same substrate using solution processing [3].

Along with the enhanced integration, effort was also made toward highly efficient parallel sensing, particularly suited for a large set of analytes and/or for redundant measurements of single analyte to improve analysis accuracy and reliability. Motivated by this goal, Cai et al [11] developed the first OLED-based multianalyte sensor platform for 
simultanesous measurement of glucose and lactate using compact integration with commercial silicon photodiodes and electronics. This platform could potentially lead to field applications in a handheld or even smaller package produced by low-cost processes. Vengasandra and Cai et al. improved the performance using a bio-CD based platform, where analyte and enzyme solutions can be injected into the reaction chambers via centrifugal force by CD-spinning. This was designed for operations similar to conventional $\mathrm{CD}$, thus loading sample into a large set of chambers becomes more efficient.

The response time of thin film PDs, techniques to monolithically fabricate and integrate all components, and miniaturization of the electronics remain the main challenges toward compact structurally integrated sensors. In regard to the PDs, reverse bias can be applied to reduce the charge collection time, therefore shortening the response time, provided that increased dark current remains significantly smaller than the signal. Nano engineering is expected to generate interdigitated donor-acceptor blend with vertical carrier transport path, which can also reduce the charge transport time of organic photodetectors (OPD). In terms of integrated fabrication of OLEDs and OPDs, inkjet printing could be feasible. The micron-size nozzle opening leads to alternating patterns of OLEDs and OPDs on the same substrate. Electronic component such as op-amp might be possible as the solution-processed CMOS technology continues to advance. It is not impractical to image that monolithically integrated all-organic solution processed sensors powered by organic solar cells based on thin films, will be ready for high volume manufacturing in the future. 


\section{Dissertation organization}

This dissertation comprises 8 chapters. The first two are an overview of OLED technology and a general introduction to OLED-based structurally integrated sensors, respectively. In Chapter 3, interface engineering techniques are investigated for enhancement of OLED performance. Chapters 4-7 are published results regarding OLED-based sensors. Chapter 4 describes the use of $\mathrm{ZnO}$ nanoparticles for enzyme immobilization. In Chapter 5, a multi-analyte sensor platform is discussed. Chapter 6 presents a PL decay curve analysis based on different models and its physics implications. Chapter 7 discusses a polymer LED and OLED-based all organic sensor platform. Finally, the general conclusions of this dissertation are summarized in Chapter 8.

\section{References}

[1] N. Tessler, N. T. Harrison, R. H. Friend, Adv. Mater., 10 (1998) 64.

[2] V. Savvate'ev, Z. Chen-Esterlit, J.W. Aylott, B. Choudahury, C.-H. Kim, L. Zou, R. Shinar, J. Shinar, R. Kopelman, Appl. Phys. Lett., 81 (2002) 4652.

[3] L. Burgi, R. Pfeiffer, M. Mucklich, P. Metzler, M. Kiy, C. Winnewisser, Organic Electronics, 7 (2006) 114.

[4] Y.-H. Kim, K.-S. Shin, J.-Y. Kang, E.-G. Yang, K.-K. Paek,D.-S. Seo and B.-K.Ju, J. Microelectromech. Syst., 15 (2006) 1152.

[5] E. Kraker, A. Haase, B. Lamprecht, G. Jakopic, Appl. Phys. Lett., 92 (2008) 033302.

[6] J. Frischeisen, N. Reinke, W. Brutting, Laser Focus World, 57 (2009).

[7] E. Ratcliff, P. A. Veneman, A. Simmonds, B. Zacher, D. Huebner, S.S. Saavedra, N. R. Armstrong, Anal. Chem., 82 (2010) 2734.

[8] Z. Zhou, R. Shinar, B. Choudhury, L.B. Tabatabai, C. Liao, J. Shinar, Proc. SPIE, 5994 (2005) 59940E-2. 
[9] R. Shinar, Z. Zhou, B. Choudhury, J. Shinar, Anal. Chim. Act., 568 (2006) 190; R.T. Cummings, S.P. Salowe, B.R. Cunningham, J. Wiltsie, Y.W. Park, L.M. Sonatore, D. Wisniewski, C.M. Douglas, J. D. Hermes, E.M. Scolnick, Proc. Nat. Acad. Sci., 99 (2002) 6603.

[10] J. R. Lakowicz, Pinciples of fluorescence spectroscopy $2^{\text {nd }}$ ed., Kluwer Academi (1999).

[11] Y. Cai, R. Shinar, Z. Zhou, J. Shinar, Sen. Actu. B, 134 (2008) 727.

[12] F. So, Organic Electronics: Materials, Processing, Devices and Applications, CRC press (2010).

[13] B. Choudhury, R. Shinar, J. Shinar, J. Appl. Phys., 96 (5) (2004) 2949.

[14] Y. Cai, R. Shinar, J. Shinar, SPIE Conf. Proc., Vol. 7418 (2009).

[15] R. Shinar, D. Ghosh, B. Choudhury, M. Noack, V. L. Dalal, J. Shinar, J. Non-crystalline Solids, 352 (2006) 1995.

[16] S. Vengasandra, Y. Cai, D. Grewell, J. Shinar, R. Shinar, Lab Chip, 10 (2010) 1051. 


\title{
Chapter 3. Interface engineering for OLED improvement
}

\begin{abstract}
Modern OLEDs are typically made of multi-layers with each layer having a specific functionality. Carrier injection, carrier transport and stability critically rely on the properties of the interfaces between neighboring layers. Electrode-organic, organic-organic, and substrate-air interfaces were engineered to improve the carrier injection as well as outcoupling for micro-cavity, ultra violet (UV), and green OLEDs. Over an order of magnitude enhancement of carrier injection was achieved in both micro-cavity and UV OLEDs. The efficiency was improved for all three cases.
\end{abstract}

\section{Introduction}

Since Tang's work published in 1987 [1], multi-layer structure for OLEDs has received intensive attention and research efforts, with each layer specifically undertaking a certain functionality, ie., hole injection, hole transport, emission, electron transport, electron injection. Energy levels (HOMOs and LUMOs) are usually not aligned from layer to layer. The significance of the interfaces between the adjacent layers has been shown, in terms of charge injection and outcoupling [2-4], which determine the overall device efficiency. Interface study also presents opportunities in device physics, new architectures, and novel materials. Based on the physical location, interfaces can be categorized into organic-organic interfaces, electrode-organic interfaces, and substrate (glass)-air interfaces. In the context of different OLED structures, these three types of interfaces are discussed in more detail. Moreover, novel engineering methods were applied to resolve the challenges at these interfaces of different OLEDs. 


\subsection{Microcavity OLEDs (electrode-organic interface)}

When OLEDs are sandwiched in a micro-cavity structure, changes occur in e.g., decay rate of spontaneous emission, and optical field distribution $[5,6]$. The former is subject to change depending on the environment of the emitting dipoles, which is also known as Purcell effect. It can be shown that the radiative decay rate is increased, if the emitting dipole is placed in a cavity structure. Appreciable increase (20\%) in intrinsic quantum yield has been reported, using a microcavity structure for OLEDs [5]. In addition, the electromagnetic field distribution is altered in the presence of microcavity, therefore the radiation mode is enhanced, owing to conversion of both organic and substrate modes into external light. This microcavity amplification shows an improvement of spatially and spectrally integrated power efficiency up to a factor of 2.3. This translates into an enhanced out-coupling efficiency, which is usually $\sim 20 \%$. Controllable narrowed emission of microcavity OLED presents a unique opportunity in structurally integrated PL-based sensors, where a minimal electroluminescence background is desired. By means of narrowed emission, the EL and PL spectra can be further separated, which renders edge filters more effective in blocking the background.

A microcavity structure requires two reflective mirrors, with one being also transmissive for light extraction. The position of the cavity modes are given by the relation:

$$
m \lambda=2 L(\lambda)
$$

where $m$ is the integer mode index and $L(\lambda)$ is the total optical thickness of the cavity, including the organic layers and the penetration into the mirrors. With thickness typically 
larger than $100 \mathrm{~nm}, \mathrm{Al}$ is commonly used as the cathode, which serves as a mirror as well. One of the existing technologies to make another mirror is to fabricate a Distributed Bragg Reflector (DBR) on the back side of the ITO. The DBR consists of alternating pairs of dielectric layers with high contrast of refractive index i.e., $\mathrm{SiO}_{2} / \mathrm{Si}_{\mathrm{x}} \mathrm{N}_{\mathrm{y}}, \mathrm{SiO}_{2} / \mathrm{TiO}_{2}$ and $\mathrm{SiO}_{2} / \mathrm{Ta}_{2} \mathrm{O}_{5}$. Fabrication is a labor intensive and multi-step process, requiring complex deposition systems, high-temperature, and successive ITO deposition [7-9]. However, a single thermally evaporated metal layer can be used instead for simplicity while not comprising the performance. Silver has high reflectivity for visible light and lowest resistivity among all the metals. Thus, it serves the purpose of both a mirror and an electrode. The total optical thickness for the case of two metal mirrors is given by

$$
L(\lambda)=\sum_{i} n_{i} L_{i}+\left|\frac{\varphi_{r}}{4 \pi} \lambda\right|+\left|\frac{\varphi_{e}}{4 \pi} \lambda\right|
$$

$n_{i}$ and $L_{i}$ are the refractive index and thickness of the organic layer. $\varphi_{r}$ and $\varphi_{e}$ are the phase shifts at non-transmissive and transmissive electrodes, respectively.

$$
\varphi=\arctan \left(\frac{2 n_{o} k_{m}}{n_{o}^{2}-n_{m}^{2}-k_{m}^{2}}\right)
$$

$n_{o}$ is the refractive index of the organic in contact with the metal electrode. $n_{m}$ and $k_{m}$ are the real and imaginary parts of the refractive index of the metal [7], respectively. The output electric field of the cavity relative to free space at a wavelength $\lambda$ along the normal direction is given by:

$$
\frac{\left|E_{c a v}(\lambda)^{2}\right|}{\left|E_{0}(\lambda)^{2}\right|}=\frac{T_{e}\left[1+R_{r}+2 \sqrt{R_{r}} \cos \left(\frac{4 \pi z}{\lambda}\right)\right]}{1+R_{r} R_{e}-2 \sqrt{R_{r} R_{e}} \cos \left(\frac{4 L L}{\lambda}\right)}
$$


e and $\mathrm{r}$ represent the mirrors, where light exits and where light does not, respectively. $\mathrm{T}$ and $\mathrm{R}$ are transmittance and reflectance. $z$ is the distance of the emitting dipole to the non-exiting mirror, including the penetration into the metal, and $L$ is the total optical thickness.

Although Ag possess both desired electrical and optical properties, its work function is low $(\sim 4.3 \mathrm{eV})[10,11]$, resulting in poor hole injection into the organic layers. The same problem was encountered at the ITO/Al-organic interfaces in the early days. Directly deposited dielectric layers, such as $\mathrm{LiF}$, semiconducting layer $\mathrm{MoO}_{3}$ and additional layers formed by oxidation on $\mathrm{Al}_{2} \mathrm{O}_{3}$ have been reported to improve either electron or hole injection $[2,12,13]$. This is a result of the formation of either an intermediate energy level or a dipole layer formed, which shifts the vacuum level. Both decrease the effective injection barrier. $\mathrm{Ag}_{2} \mathrm{O}$ is a p-type semiconductor with work function $\sim 1 \mathrm{eV}$ higher than that of $\mathrm{Ag}$ [14]. Thus, $\mathrm{Ag}_{\mathrm{x}} \mathrm{O}$ made by simple UV-Ozone treatment could potentially enhance the hole injection. This method was explored and the results are shown in the following section.

\subsection{UV OLEDs (organic-organic interface)}

UV OLEDs can be utilized in structurally integrated and field deployable fluorescent sensors in medical, chemical, and biological areas. They are particularly helpful for sensor materials with strong UV absorption, such as PtOEP (its $\sim 380 \mathrm{~nm}$ absorption peak is 5 times stronger than that of the $535 \mathrm{~nm}$ absorption band). Another application is to generate RGB colors through energy transfer for display and solid state lighting purposes. UV OLED poses a challenge to search high band-gap luminescent materials, which are hard to synthesize and their availability is limited. 4,4'-Bis(carbazol-9-yl)biphenyl (CBP), a common host material with a high HOMO-LUMO gap $(\sim 3.5 \mathrm{eV})$ can be used for UV emission as reported by Zou et 
al. [15]. However, the HOMO level of the stabilized hole-injection contact $\mathrm{CuPc}$ is about 1.5 $\mathrm{eV}$ lower than that of $\mathrm{CPB}$, leading to poor hole injection as shown in the energy diagram in Fig. 1.

An additional drawback of the large injection barrier is accumulation of charges, which could generate a high local field that will quench the excitons. A common hole transport layer such as NPD cannot be used to alleviate this problem, due to its luminescence nature and favored exciton transfer toward it. Consequentially, our options are very limited.

Graded junctions at the organic-organic interface have been reported to enhance the carrier injection as well as the device performance [16-19]. They can be achieved by interdiffusion of neighboring layers facilitated by post-annealing above the glass transition temperature $T_{g}$ and co-deposition of two sources or two materials in one source. Almost all the effort focused on grading the HTL and ETL, which also serves as the EML. Although there is a reported increase in the efficiency of devices prepared by annealing $[16,17]$ in simple two layer structure, the concern remains that in more complex structures, post annealing could damage other layers, especially the doped emitting layer. For graded junctions made by co-deposition, the efficiency was not improved, although the durability was enhanced $[18,19]$. It may be attributed to extended recombination zone formed by grading across the device from HTL to ETL, which makes the excitons susceptible to electrode quenching.

In this work, only the HTL/EML interface was graded. It was realized by 4 steps with the graded compositional change confined within a thickness of up to $20 \mathrm{~nm}$ in UV OLEDs. The IV characteristic was studied as well as the external quantum efficiency. Comparison is 
made for devices with and without graded junction as well as for devices with graded junction of different thicknesses.

\subsection{Alq $_{3}$-based OLEDs (glass-air interface)}

Only $\sim 20 \%$ of the photons generated within the device can be coupled out, partially due to total internal reflection that occurs at the glass/air interface $\left(\mathrm{n}_{\text {glass }}=1.5 ; \mathrm{n}_{\text {air }}=1\right)[20]$. By outcoupling more waveguided photons, devices can be driven at a lower current for the same brightness, leading to reduced degradation and extended device lifetime. This is crucial for commercial applications, where stable performance is desired, such as display, solid state lighting and OLED-based sensors.

It has been observed that outcoupling can be enhanced up to $\sim 50 \%$ using microlens made by imprint lithography, micro-contact printing, and etching as well as by using textured surface [21-24]. However, high temperature, multi-step processes or complex chemistry are required, resulting in high fabrication cost. Chen et al. reported $\sim 20 \%$ increase by sandblasting the edge and back-side of the glass substrates [25]. Although this is a low cost and efficient process, the size of the sand particles limits the average grain size, which is $100 \mu \mathrm{m}$. It also suggested that the use of smaller grain sizes should further increase the scattering probability.

A novel low-cost simple casting approach to roughen the glass substrate was tested. Cheap materials were used such as index matching polymer (polystyrene (PS)) and nanoparticles $\left(\mathrm{TiO}_{2}\right)$, which are manufactured at large scale and widely used in the paint 
industry. Particles size can be chosen for optimized scattering at a certain wavelength. Device improvement in green OLEDs and challenges are discussed in the results section.

\section{Experimental procedures and device structures}

General OLED fabrication procedure starts with ITO cleaning followed by thermal evaporation of the organic layers and cathode. Deposition was done in a high vacuum chamber $\left(<10^{-6}\right.$ mbar $)$ housed in an Ar-filled glovebox. Devices were encapsulated after fabrication for protection from $\mathrm{O}_{2}$ and $\mathrm{H}_{2} \mathrm{O}$ during characterization. For microcavity OLEDs, the starting substrate is just a glass slide. The anode silver was thermally evaporated through a shadow mask. A graded junction was achieved by 4 steps, with each one having a mixed layer, which has a gradual compositional change with decreasing $\mathrm{CuPc}$ and increasing $\mathrm{CBP}$ from the HTL to the EML. The pure CuPc is followed by $80 \% \mathrm{CuPc}: 20 \% \mathrm{CBP}, 60 \%$ CuPc:40\% CBP, 40\% CuPc:60\% CBP, 20\% CuPc:80\% CBP and pure CBP (w\%). The 4 sub-interfacial layers have the same thicknesses. Spatially and spectrally integrated spectra were measured for a green OLED pixel. Then $3 \mu \mathrm{L}$ of toluene solution containing $2 \mathrm{mg} / \mathrm{mL}$ $\mathrm{TiO}_{2}$ and $40 \mathrm{mg} / \mathrm{mL}$ polystyrene was dropcast on top of the glass side of the pixel. After 12 hours, the integrated spectrum on the same pixel was measured again using the same set-up. The area under the spectrum is proportional to the total number of the outcoupled photons. Therefore, the ratio of the spectral integrations yields the outcoupling enhancement.

\subsection{Microcavity OLEDs:}

Ag 26.5 nm/UV-Ozone (x minutes)/CuPc 6 nm/NPB 80 nm/Alq 70nm/LiF $1 \mathrm{~nm} / \mathrm{Al} 120 \mathrm{~nm}$ Device A: 0 minute, Device B: 1 minute, Device C: 3 minutes 
Device E: Ag $26.5 \mathrm{~nm} / \mathrm{UV}-$ Ozone 3 minutes/CuPc $5 \mathrm{~nm} / \mathrm{NPD} 75 \mathrm{~nm} / \mathrm{Alq}_{3} 75 \mathrm{~nm} / \mathrm{LiF} 1$ $\mathrm{nm} / \mathrm{Al} 136 \mathrm{~nm}$

Device F: ITO/CuP 5 nm/NPD 50 nm/Alq $350 \mathrm{~nm} / \mathrm{LiF} 1 \mathrm{~nm} / \mathrm{Al} 136 \mathrm{~nm}$

\subsection{UV OLEDs:}

ITO/CuPc 15 nm/Graded CuPc:CBP x nm/CBP y nm/BCP 20 nm/Alq 10 nm/CsF $1 \mathrm{~nm} / \mathrm{Al}$ $125 \mathrm{~nm}$

Device A: $\mathrm{x}=20 \mathrm{~nm}, \mathrm{y}=30 \mathrm{~nm}$

Device B: $\mathrm{x}=10 \mathrm{~nm}, \mathrm{y}=40 \mathrm{~nm}$

Device C: $\mathrm{x}=5 \mathrm{~nm}, \mathrm{y}=45 \mathrm{~nm}$

Device D: $\mathrm{x}=0 \mathrm{~nm}, \mathrm{y}=50 \mathrm{~nm}$

\subsection{Alq $_{3}$-Based OLEDs:}

Device A: glass $/ \mathrm{ITO} / \mathrm{MoO}_{3} 5 \mathrm{~nm} / \mathrm{NPD} 50 \mathrm{~nm} / \mathrm{Alq}_{3} 50 \mathrm{~nm} / \mathrm{LiF} 1 \mathrm{~nm} / \mathrm{Al} 100+\mathrm{nm}$

Device A': nanoparticles/glass/ITO/MoO 3 nm/NPD $50 \mathrm{~nm} / \mathrm{Alq}_{3} 50 \mathrm{~nm} / \mathrm{LiF} 1 \mathrm{~nm} / \mathrm{Al}$ 100+ $\mathrm{nm}$

\section{Results and discussion}

\subsection{Microcavity OLED (electrode-organic interface)}

As shown in Fig. 1a, the current density is only $\sim 2 \mathrm{~mA} / \mathrm{cm}^{2}$ at $30 \mathrm{~V}$ for untreated $\mathrm{Ag}$, while the 1 minute UV-Ozone treatment increases the current density to $\sim 20 \mathrm{~mA} / \mathrm{cm}^{2}$ and 3 minutes treatment enhances to $\sim 50 \mathrm{~mA} / \mathrm{cm}^{2}$. This is attributed to the $\mathrm{Ag}_{\mathrm{x}} \mathrm{O}$ layer, produced by UV-Ozone treatment. 3 minutes treatment results in better performance than 1 minute, probably due to better $\mathrm{Ag}_{\mathrm{x}} \mathrm{O}$ coverage on $\mathrm{Ag}$. 
The efficiency measured along the normal direction is shown in Fig. 2b. At a current density of $1 \mathrm{~mA} / \mathrm{cm}^{2}$, the efficiency increased from $0.1 \mathrm{Cd} / \mathrm{A}$ to $0.4 \mathrm{Cd} / \mathrm{A}$ for 1 minute treatment and to $0.8 \mathrm{Cd} / \mathrm{A}$ for 3 minute treatment, which can be attributed to enhanced holeelectron balance at the recombination zone due to increased hole injection. However, the current increase is larger than the efficiency increase. This may indicate that the injected holes outnumber the electrons. Thus, improvement in electron injection and transport could further enhance the efficiency.

It should be noted that the applied voltage is high compared to conventional OLEDs at the same current density. In addition to the poor injecting contact, the total optical thickness was $\sim 1.5$ fold thicker to have a cavity mode close to the intrinsic PL, which resulted in 50\% increase in series resistance. Ag-based microcavity OLED with UV-Ozone treatment (device E) shows much narrowed spectrum compared to conventional structures (device F) as shown in Fig.3. The full width half maximum shrunk from $94 \mathrm{~nm}$ to $19 \mathrm{~nm}$, leading to $\mathrm{Q}$ factor $\left(\frac{\lambda}{\Delta \lambda}\right)$ increase from 5.5 to 28 , which demonstrates a strong microcavity effect.

\subsection{UV OLEDs (organic-organic interface)}

Figure 4 shows the IV-characteristics of CBP-based UV OLED with graded interfacial layers of different thicknesses and abrupt junctions. At $14 \mathrm{~V}$, the current density increases from $\sim 3 \mathrm{~mA} / \mathrm{cm}^{2}$ for abrupt junction to $\sim 40 \mathrm{~mA} / \mathrm{cm}^{2}$ for $20 \mathrm{~nm}$ thick graded junction. Concomitantly, the external quantum efficiency is increased from $0.4 \%$ to $0.65 \%$. There are two possible explanations for the increased current density. Physically, in the 
graded interfacial layer, the effective contract area of $\mathrm{CuPc}$ and $\mathrm{CBP}$ is increased, thus for every single hole residing on a site of $\mathrm{CuPc}$, there are more available sites on $\mathrm{CBP}$ to hop to. This can be visualized by considering the microscopic phase segregation. Two phases are percolated separately, extending oppositely to the pure layers. Energetically, the intermixing induces structural disorder, which broadens the density of transport states. Therefore the effective energy barrier is lowered considering the extension of the tails states. This is equivalent to that the effective HOMO of each sub-interfacial layer becomes higher from $\mathrm{CuPc}$ to $\mathrm{CBP}$, thus energy ladders are provided for sequential small jumps instead of a big one. This can be better understood by means of tunneling theory. The relation of tunneling current is proportional to $\exp \left(-K \phi^{\frac{3}{2}}\right)$, here $\phi$ is the injection barrier. The current for step barriers is proportional to

$$
\begin{gathered}
\exp \left(-K \phi_{1}^{\frac{3}{2}}\right) \times \exp \left(-K \phi_{2}^{\frac{3}{2}}\right) \times \exp \left(-K \phi_{3}{ }^{\frac{3}{2}}\right) \times \exp \left(-K \phi_{4}{ }^{\frac{3}{2}}\right)= \\
\exp \left(-K\left(\phi_{1}^{\frac{3}{2}}+\phi_{2}{ }^{\frac{3}{2}}+\phi_{3^{\frac{3}{2}}}+\phi_{4}^{\frac{3}{2}}\right)\right)>\exp \left(-K\left(\phi_{1}+\phi_{2}+\phi_{3}+\phi_{4}{ }^{3 / 2}\right)\right. \\
\phi_{1}+\phi_{2}+\phi_{3}+\phi_{4}=\phi, \text { the single barrier height }
\end{gathered}
$$

Thicker graded junction is suspected to induce more microscopic phase segregation with each phase connected continuously in a complex manner, leading to enlarged contact area. Also it could generate more inter-diffusion of neighboring sub-interfacial layers, resulting in higher degree of grading. As a result, the number of energy steps for injection is increased, which increases the injection current, based on the aforementioned tunneling theory. 


\subsection{Nanoparticles (glass-air interface)}

Emission spectra were measured for the same device at the same driving current before and after casting the functional layer made of $\mathrm{TiO}_{2}: \mathrm{PS}$ nanoparticles, using an

integrating sphere. As shown in Fig. 5, with $\mathrm{TiO}_{2}$ layer, the integrated spectrum increases by $\sim 6 \%$, which indicates that the out-coupling is enhanced by $6 \%$. The thickness of the $\mathrm{TiO}_{2}: \mathrm{PS}$

film was about $\sim 5 \mu \mathrm{m}$, which is equivalent to several layers of $\mathrm{TiO}_{2}$ nanoparticles having diameter of $\sim 360 \mathrm{~nm}$. The refractive index of polystyrene is 1.55 , very close to that of the glass substrate, which is 1.52 . Therefore the photons arriving at the glass/polymer interface experience little loss due to reflection and no total internal reflection is expected. Before they reach the top layer of the polymer, strong scattering occurs and it does not necessarily help extract the photons toward the polymer/air interface. Once the photons reach the top layer, the nanoparticles perform as microlens, which have been reported to enhance the outcoupling. The observed $6 \%$ net enhancement is expected to be further improved using a monolayer of $\mathrm{TiO}_{2}$ doped polymers with matched index.

\section{Conclusions}

UV-Ozone treatment and step-graded heterojunctions have been shown in silverbased microcavity OLEDs and CBP-based UV OLEDs to alter the charge injection barrier, resulting in improved current by over an order of magnitude. Efficiency enhancement has also been observed in both cases. Nanoparticle doped polymer film has been demonstrated to improve the out-coupling efficiency by $\sim 6 \%$. Further increase is expected by making a monolayer of the particles. 


\section{References}

[1] C.W. Tang, S.A. VanSklyke, Appl. Phys. Lett., 51(2) (1987) 913.

[2] L.S. Hung, C.W. Tang, M. G. Mason, Appl. Phys. Lett., 70 (2) (1997) 152.

[3] S.A. Van Slyke, C.H. Chen, C.W. Tang, Appl. Phys. Lett., 69 (15) (1996) 2160.

[4] Y. Sun, S.R. Forrest, J. Appl. Phys., 100 (2006) 073106.

[5] Q. Huang, S. Reineke, K. Walzer, M. Pfeiffer, K. Leo, Appl. Phys. Lett., 89 (2006) 263512.

[6] R. Meerheim, R. Nitsche, K. Leo, Appl. Phys. Lett., 93 (2008) 043310.

[7] A. Dodabalapur, L.J. Rothberg, R. H. Jordan, T. M. Miller, R. E. Slusher, J. M. Philips, J. Appl. Phys., 80(12) (1996) 6954.

[8] S. Tokito, T. Tsutsui, Y. Taga, J. Appl. Phys., 86(5) (1999) 2407.

[9] F. Ma, X. Liu, C. Zhang, H. Li, L. Wang, Jap. J. Appl. Phys., 45(12) (2006) 9224.

[10] C. W. Chen, P. Y. Hsieh, H. H. Chiang, C. H. Lin, H. M. Wu, and C. C. Wu, Appl. Phys. Lett., 83 (2003) 5127.

[11] Y. Q. Li, J. X. Tang, Z. Y. Xie, L. S. Hung, and S. S. Lau, Chem. Phys. Lett., 386 (2004) 128.

[12] H. You, Y. Dai, Z. Zhang, D. Ma, J. Appl. Phys., 101 (2007) 026105.

[13] H. Tang, F. Li, J. Shinar, Appl. Phys. Lett., 71 (18) (1997) 2560.

[14] H. W. Choi, S. Y. Kim, K. Kim, Y. Tak, J. Lee, Appl. Phys. Lett., 86 (2008) 012104.

[15] L. Zou, V. Savvate'ev, J. Booher, C. Kim, J. Shinar, Appl. Phys. Lett., 79(14) (2001) 2282.

[16] C. Chen, T. Cho, C. Wu, H. Yu, T. Luh, Appl. Phys. Lett., 81(9) (2002) 1570.

[17] B.J. Chen, X.W. Sun, T.K.S. Wong, X. Hu, A. Uddin, Appl. Phys. Lett., 87 (2005) 063505.

[18] A.B. Chwang, R. C. Kwong, J.J. Brown, Appl. Phys. Lett., 80(5) (2002) 725. 
[19] Y. Shao, Y. Yang, Appl. Phys. Lett., 83(12) (2003) 2453.

[20] C. Adachi, M.A. Baldo, M.E. Thompson, S.R. Forrest, J. Appl. Phys., 90(10) (2001) 5048.

[21] Y. Sun, S. R. Forrest, J. Appl. Phys., 100 (2006) 073106.

[22] F. Li, X. Li, J. Zhang, B. Yang, Organic Electronics, 8 (2007) 635.

[23] H. J. Peng, Y.L. Ho, C.F. Qiu, M. Wong, H.S. Kwok, SID, 11.4 (2004) 158.

[24] Y. Cheng, J. Wu, C. Cheng, K. Syao, M. Lee, Appl. Phys. Lett., 90 (2007) 091102.

[25] S. Chen, H. Kwok, Opti. Exp., 18(1) (2010) 37. 
Figures

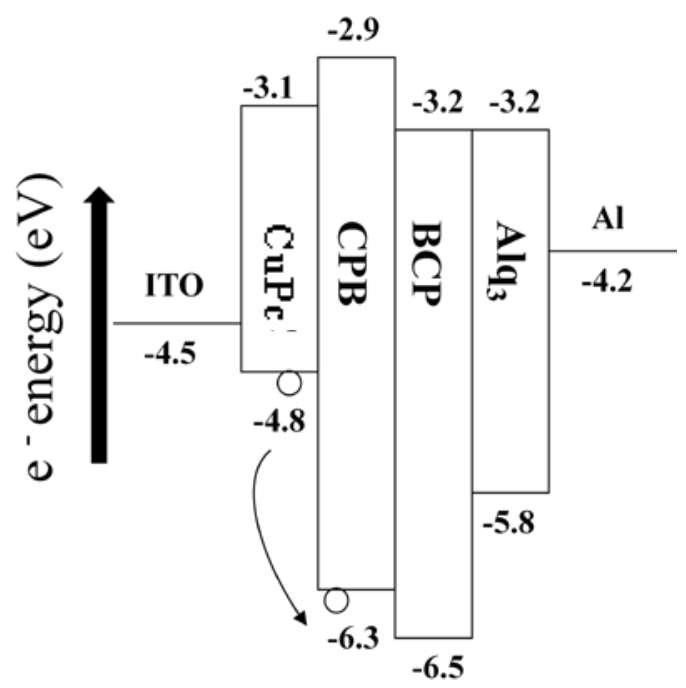

Fig. 1. Energy diagram of CBP-based UV OLED 

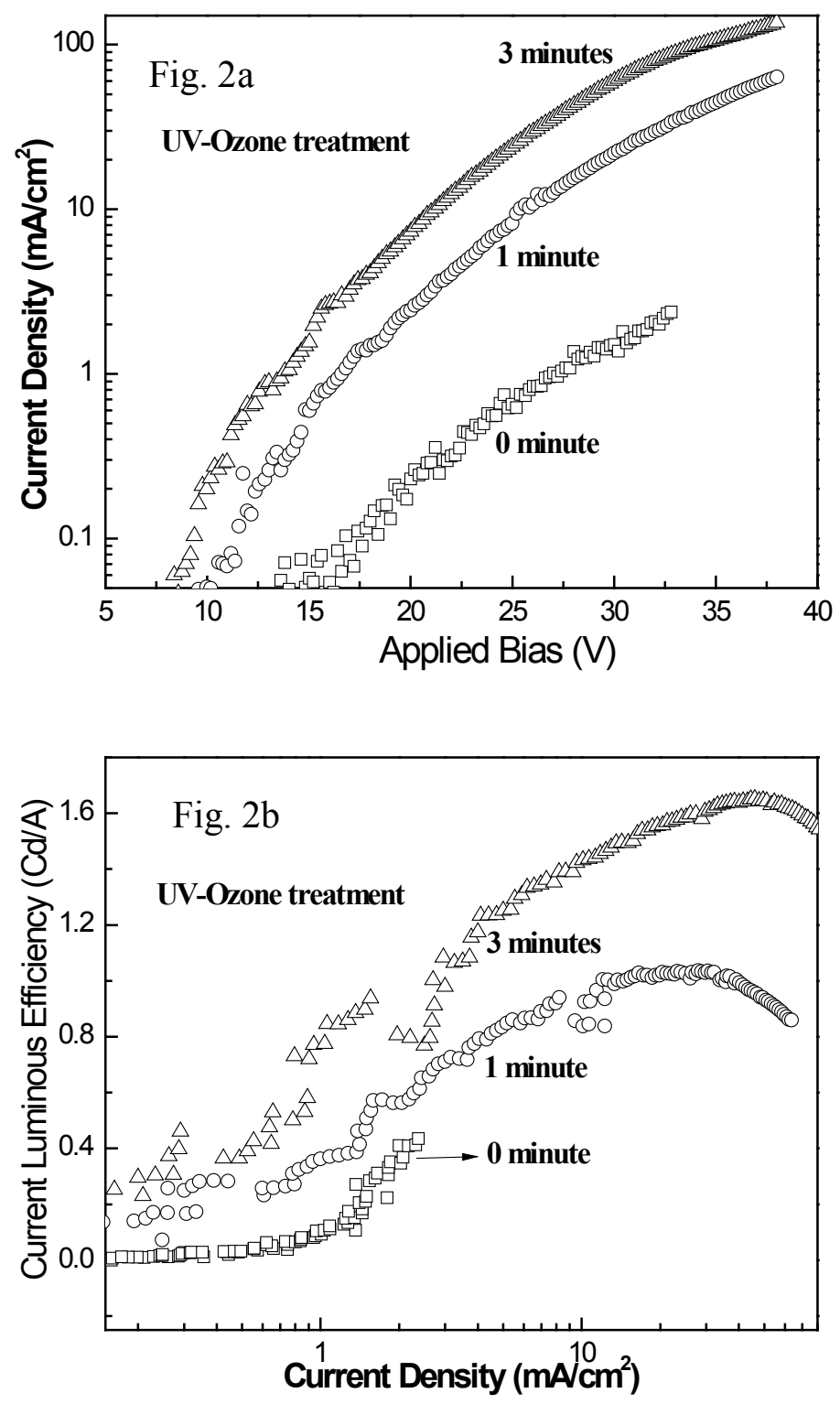

Fig.2. IV characteristic (2a) and efficiency vs current (2b) for devices with different UVOzone treatment time 


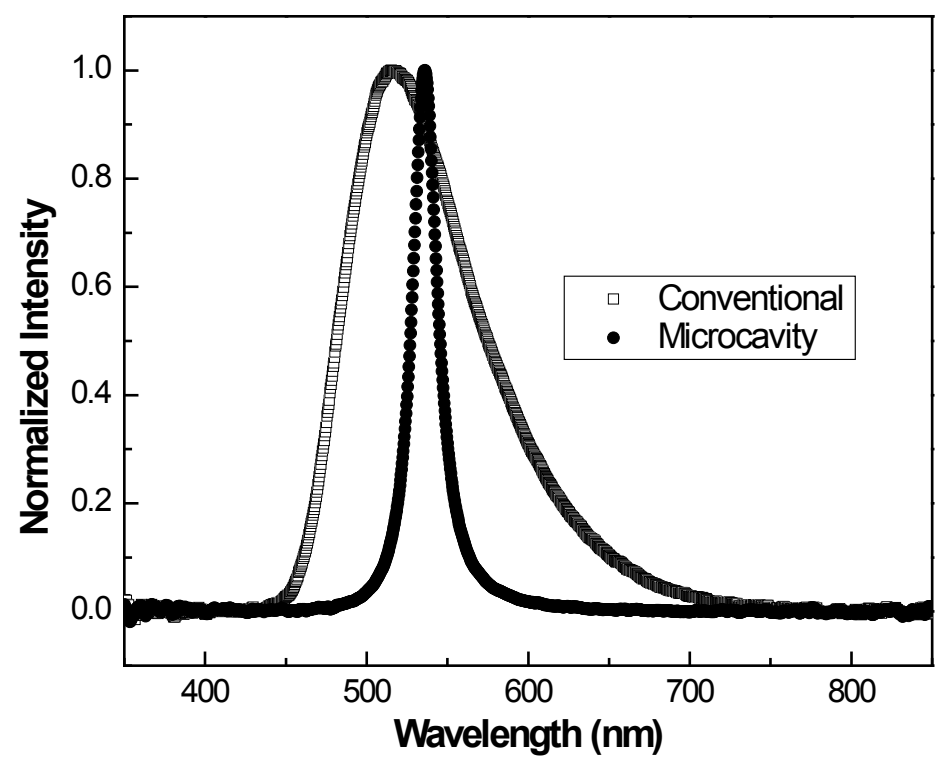

Fig. 3. Spectra for $\mathrm{Alq}_{3}$-based microcavity and conventional OLEDs 

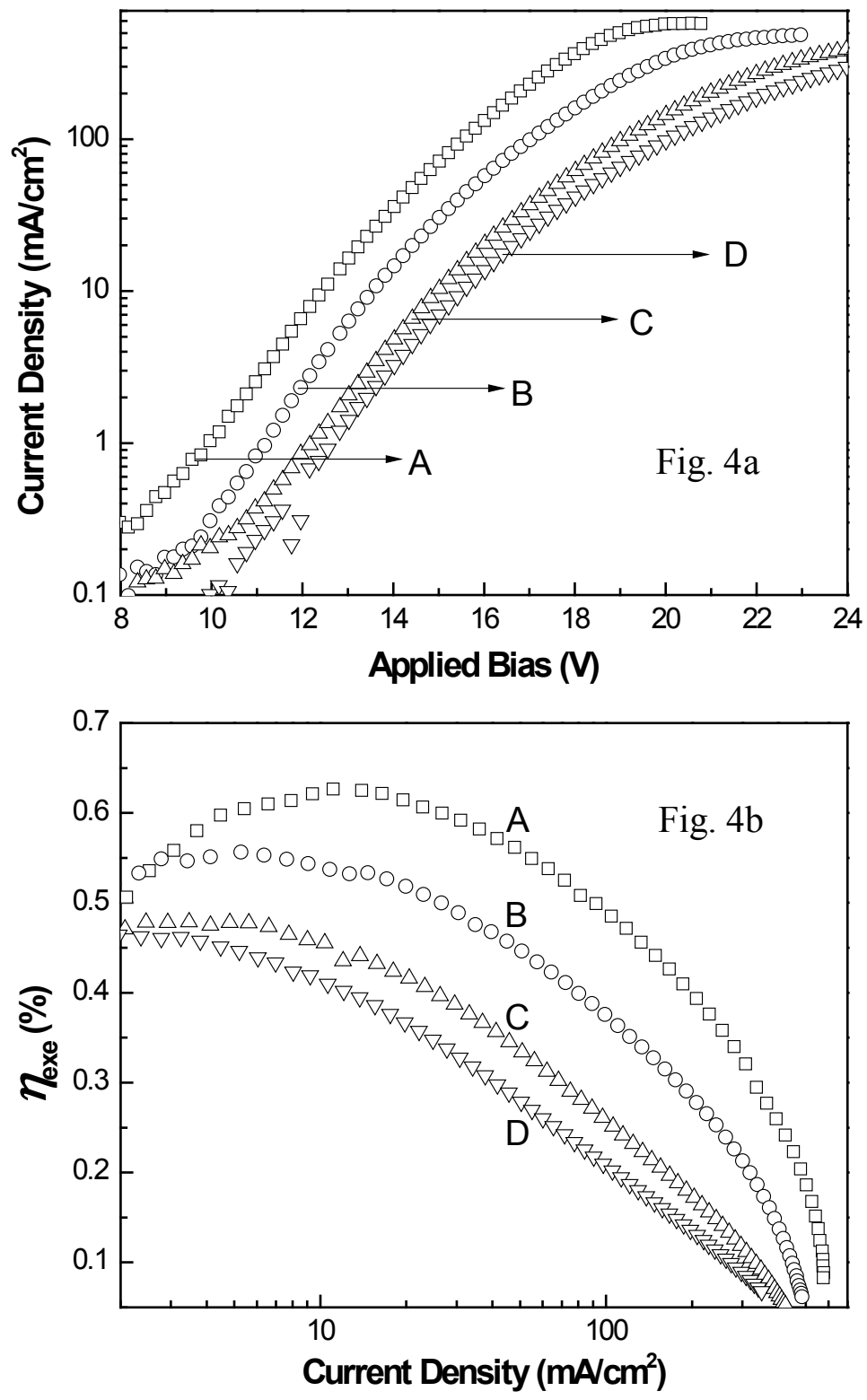

Fig. 4. IV characteristic (4a) and EQE vs current (4b) for devices with different thicknesses of graded junction (A: $20 \mathrm{~nm}, \mathrm{~B}: 10 \mathrm{~nm}, \mathrm{C}: 5 \mathrm{~nm}, \mathrm{D}: 0 \mathrm{~nm}$ ) 


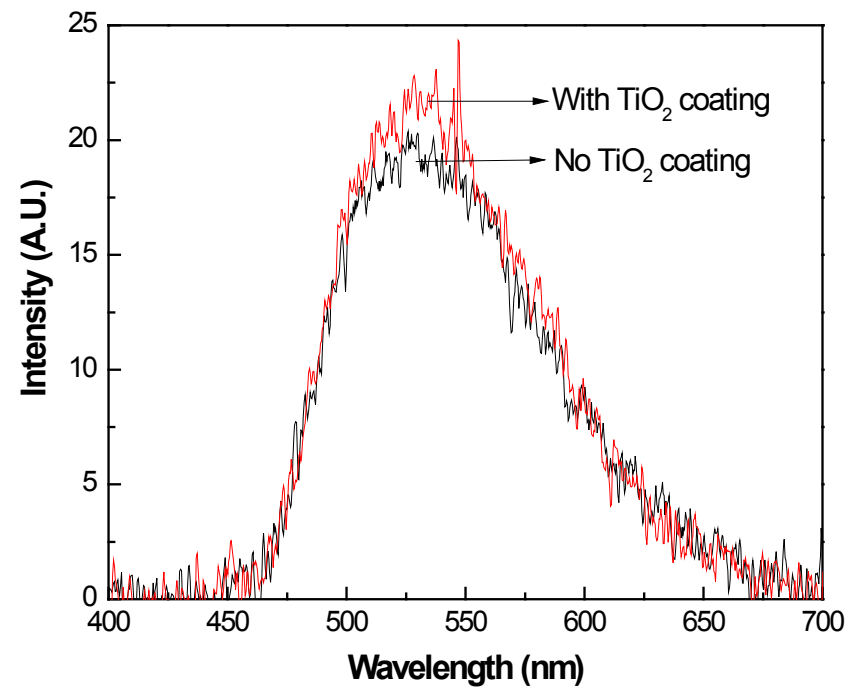

Fig. 5. Spectra of the same $\mathrm{Alq}_{3}$-based OLED with and without $\mathrm{TiO}_{2}$ : polystyrene coating measured by integrating sphere 


\title{
Chapter 4. OLED-based biosensing platform with $\mathrm{ZnO}$ nanoparticles for enzyme immobilization
}

A paper published in SPIE proceedings, 7418, 74180R (2009)

\author{
Y. Cai, R. Shinar, J. Shinar
}

\begin{abstract}
Organic light-emitting diode (OLED)-based sensing platforms are attractive for photoluminescence (PL)-based monitoring of a variety of analytes. Among the promising OLED attributes for sensing applications is the thin and flexible size and design of the OLED pixel array that is used for PL excitation. To generate a compact, field-deployable sensor, other major sensor components, such as the sensing probe and the photodetector, in addition to the thin excitation source, should be compact. To this end, the OLED-based sensing platform was tested with composite thin biosensing films, where oxidase enzymes were immobilized on $\mathrm{ZnO}$ nanoparticles, rather than dissolved in solution, to generate a more compact device. The analytes tested, glucose, cholesterol, and lactate, were monitored by following their oxidation reactions in the presence of oxygen and their respective oxidase enzymes. During such reactions, oxygen is consumed and its residual concentration, which is determined by the initial concentration of the above-mentioned analytes, is monitored. The sensors utilized the oxygen-sensitive dye Pt octaethylporphyrin, embedded in polystyrene. The enzymes were sandwiched between two thin $\mathrm{ZnO}$ layers, an approach that was found to improve the stability of the sensing probes.
\end{abstract}




\section{Introduction}

The field of OLED-based chemical and biological sensors has been growing rapidly over the past 5 years [1-6]. In addition to the electronics and read-out components, such sensors comprise excitation sources, which are OLED pixels, sensing probes, e.g., thin polystyrene (PS) films with an embedded oxygen sensitive dye such as Pt octaethylporphyrin (PtOEP), and a photodetector (PD). The OLEDs are intrinsically thin $(<0.5 \mu \mathrm{m}$ excluding the glass substrate on which they are fabricated), compatible with flexible substrates and microfluidic structures, easy to fabricate, and their pixels are individually addressable. These attributes make OLEDs promising candidates for structurally integrated and compact photoluminescence (PL)-based sensors. Unlike other excitation sources, including inorganic LEDs, OLEDs do not require the addition of optical components, such as optical fibers or lens, and they can be fabricated on glass or plastic substrates.

The oxygen sensor is one of the most studied among OLED-based PL sensors [1, 36]. Biological samples, such as glucose, lactate, and cholesterol, which are major constituents of blood serum and have an important physiological impact on the human body, react with $\mathrm{O}_{2}$ in the presence of their respective oxidases, thus changing the dissolved oxygen (DO) level in the vicinity of the oxygen-sensitive dye molecules. The PL decay time $\tau$ or PL intensity $I$ of the dye molecules under different degrees of oxygen quenching are used for determining the DO level [DO], which is governed by the initial analyte concentration $[\text { analyte }]_{\text {initial. }}$ Structural simplicity and compactness can be enhanced by immobilizing the oxidases in a thin film, which is readily integrated with the PtOEP film. Progress in this regard has been demonstrated by immobilizing GOx in a sol-gel matrix [1]. However, recent 
studies [6], including for simultaneous monitoring of multiple analytes, utilized enzymes dissolved in solutions, which renders the sensor less compact, diminishing one of the most significant OLED attributes, i.e., its thinness and flexible size and design.

Nanostructured $\mathrm{ZnO}$ has unique advantages for immobilizing enzymes. $\mathrm{ZnO}$ is a 3.37 eV wide gap semiconductor and the nanostructured material has a large specific surface area. Other attributes include nontoxicity, chemical stability, and good biological compatibility [717]. Importantly, $\mathrm{ZnO}$ has a high isoelectric point (IEP) 9.5 [18]. Oxidases of low IEP can be adsorbed onto the surface of $\mathrm{ZnO}$, assisted by electrostatic attraction between the positively charged $\mathrm{ZnO}$ surface and the negatively charged oxidase, in solutions of proper $\mathrm{pH}$. To date, various forms of $\mathrm{ZnO}$ nanostructures have been reported to successfully immobilize enzymes in amperometric sensors. These structures include nanorods [7, 14-16], nanocombs [10], nanoparticles [12], nanocomposites [13, 17], nanoporous thin films [11], and pyramid-shaped nanoporous $\mathrm{ZnO}[8]$.

Amperometric sensors with $\mathrm{ZnO}$ have been demonstrated for detection of glucose, cholesterol, $\mathrm{H}_{2} \mathrm{O}_{2}$, uric acid, urea, and phenol [7-17]. However $\mathrm{ZnO}$ nanostructures have not been applied extensively in optical sensors. In this paper, $\mathrm{ZnO}$-assisted immobilization is investigated for the first time in a PL-based sensor excited by an OLED, providing the first steps for generating thin-film based sensor probes, to further improve the platform robustness and reduce the overall sensor size. The IEPs of glucose oxidase (GOx), stabilized lactate oxidase (SLOx), and cholesterol oxidase (ChOx) used in this work are $4.2, \sim 4.6$, and $~ 5.5$, respectively [19], resulting in easy immobilization on the-nano $\mathrm{ZnO}$ surface. Multi-step and labor-intensive procedures, such as hydrothermal $[7,14,15]$, thermal evaporation $[9,16,22]$, vapor phase transport [10], aqueous solution processing [12], RF sputtering [11] and sol-gel 
$[20,21]$, have been used to fabricate the $\mathrm{ZnO}$ nanostructures. To simplify fabrication and make it compatible with the fabrication procedure of the $\mathrm{O}_{2}$ sensor film, in this study, commercially available $\mathrm{ZnO}$ nanoparticles are dropcast directly on the PtOEP:PS sensor film. The enzyme was immobilized on the $\mathrm{ZnO}$ particles and, in an attempt to prevent it from leaching, a thin nanoparticle-based $\mathrm{ZnO}$ protective capping layer was also applied.

The biosensing platform was based on an array of OLEDs pixels, with electroluminescence (EL) that peaks at $\sim 525 \mathrm{~nm}$, which excites the PtOEP molecules embedded in the PS matrix. The PtOEP PL, which peaks at $\sim 645 \mathrm{~nm}$, is dynamically quenched by the oxygen molecules. The relation between $I$ and $\tau$ and the oxygen concentration $\left[\mathrm{O}_{2}\right]$ is ideally given by Stern-Volmer (SV) relation:

$$
I_{0} / I=\tau_{0} / \tau=1+K_{S V}\left[\mathrm{O}_{2}\right]
$$

where $I_{0}$ and $\tau_{0}$ are the unquenched values, and $K_{\mathrm{sv}}$ is the SV constant, which is film and temperature dependent. The sensor films used in this study contained additionally $\mathrm{TiO}_{2}$ particles that resulted in enhanced PL. ${ }^{5}$ Results on thin films made of PtOEP:TiO $2: \mathrm{PS} / \mathrm{ZnO} /$ oxidase/ZnO for glucose, cholesterol, and lactate monitoring are reported in the following sections; capping with $\mathrm{ZnO}$ enabled the use of these composite films. Either $\tau$ or $I$ can be used to evaluate the oxygen concentration, however, the use of $\square$ is advantageous as it is independent of variations in the excitation and background light intensities, and minor degradation of the OLED and dye, thus eliminating the need for frequent sensor calibration. OLED pulsing is required for the $\tau$ mode of operation, which reduces the OLED-on time, extending its operational lifetime. The relatively long $\tau$ of PtOEP $\left(\sim 100 \mu\right.$ s in the absence of $\left.\mathrm{O}_{2}\right)$ renders this mode attractive due to the ease of measurement; 
the long $\tau$ results in better detection sensitivity. The $\tau$ values were therefore monitored throughout this work to evaluate the performance of the OLED-based $\mathrm{ZnO}$-assisted biosensing platform.

\section{Experimental}

\subsection{Materials}

OLEDs. $20 \Omega / \square$ ITO was obtained from Colorado Concept Coatings. Copper phthalocyanine $(\mathrm{CuPc})$ and $\mathrm{LiF}$ were obtained from Sigma-Aldrich. N,N'-diphenyl-N,N'bis(1-naphthyl phenyl)-1,1'-biphenyl-4,4'-diamine ( $\alpha$-NPD), 2,3,6,7-Tetrahydro-1,1,7,7,tetramethyl- 1H,5H,11H-10-(2- benzothiazolyl) quinolizino-[9,9a,1gh] coumarin (C545T), and tris(quinolinolate) $\mathrm{Al}\left(\mathrm{Alq}_{3}\right)$ were obtained from H.W. Sands.

Sensing probes and analytes. $\mathrm{ZnO}$ nanoparticles (average size $<100 \mathrm{~nm}$ or $50 \mathrm{~nm}$ ), GOx from Aspergillus niger, ChOx from Pseudomonas fluorescens, cholesterol, and lithium 1-lactate were obtained from Sigma-Aldrich. Glucose was obtained from Fisher Scientific. SLOx from Aerococcus viridans was obtained from Applied Enzyme Technology. PtOEP was obtained from H. W. Sands. PS (average molecular weight $\sim 45,000$ ) was obtained from Sigma-Aldrich. $\mathrm{TiO}_{2}$ nanoparticles $(\sim 360 \mathrm{~nm}$ in diameter on average $)$ were obtained from $\mathrm{Du}$ Pont. Triton X-100 was obtained from Fisher Scientific.

\subsection{OLED fabrication}

Green $(\sim 525 \mathrm{~nm})$ emitting OLED pixels were fabricated by thermally evaporating organic materials on $\sim 150 \mathrm{~nm}$ thick cleaned and UV ozone-treated ITO-coated glass. The organic layers, in sequence, are the $\sim 5 \mathrm{~nm}$ hole injection layer $\mathrm{CuPc}, \sim 50 \mathrm{~nm}$ hole transport layer, $\alpha-\mathrm{NPD}, \sim 20 \mathrm{~nm}$ doped emitting layer C545T:Alq $(1 \% \mathrm{v} / \mathrm{v})$, and $\sim 30 \mathrm{~nm}$ electron 
transport layer $\mathrm{Alq}_{3}$, which is followed by a $\sim 1 \mathrm{~nm}$ electron injection layer $\mathrm{LiF}$ and the $\sim 100$ nm Al cathode. The device was encapsulated with cover glass glued by Torr Seal to prevent exposure to water and oxygen. OLED pixels were generated by etching the ITO into two 2 $\mathrm{cm}$ wide strips; the OLED pixels are defined by the overlapping regions of mutually perpendicular ITO and Al strips. Two OLED pixels $\left(2 \times 2 \mathrm{~mm}^{2}\right)$ were used as the excitation source for the PL measurements.

\subsection{Analyte and oxidase solutions}

Phosphate buffer ( $\mathrm{pH}$ 7.4) was prepared with deionized (DI) water. Glucose, lactate, and all the oxidases were dissolved in the buffer at the desired concentrations. Cholesterol was dissolved in Triton X-100; it was heated up to $\sim 40{ }^{\circ} \mathrm{C}$ until the solution was clear. The Triton solution was then mixed with the buffer using Vortex until a uniform and clear solution was obtained. The final concentration of the Triton X-100 in the buffer solution was $10 \% \mathrm{v} / \mathrm{v}$. All the analytes and oxidases buffered solutions were stored at $-4{ }^{\circ} \mathrm{C}$ when not in use.

\subsection{Sensor film fabrication}

$20 \mu \mathrm{L}$ toluene solutions containing PtOEP:PS:TiO $2(1: 1: 40 \mathrm{mg} / \mathrm{mL})$ mixtures were dropcast onto a glass slide and allowed to dry at room temperature in the dark for $\sim 24 \mathrm{~h}$ to form the oxygen sensing layer, which was $\sim 8 \mathrm{~cm}$ in diameter and $6-8 \mu \mathrm{m}$ thick. Due to the high dielectric contrast between $\mathrm{TiO}_{2}$ nanoparticles and the polymer film, strong scattering of the EL occurs, resulting in increased optical path within the film and consequently increased absorption by the dye, which enhances $I$ significantly. ${ }^{5}$ The sandwiched enzyme layer was fabricated by first dropcasting $\sim 15 \mu \mathrm{L} \mathrm{ZnO}$ in ethanol suspension onto the PtOEP:TiO $2: \mathrm{PS}$ layer, then drying it in air, followed by dropcasting $\sim 40 \mu \mathrm{L}$ of oxidase solution, and drying it 
at $-4{ }^{\circ} \mathrm{C}$. Finally, another $15 \mu \mathrm{L} \mathrm{ZnO} /$ ethanol suspension was dropcast onto the enzyme to hinder enzyme leaching.

\subsection{Instrumentation}

The biosensing platform was configured in the back detection mode [1, 3]. To minimize interference from the 'delayed' EL, which is largely due to radiative recombination of detrapped charges in the OLED after the driving pulse is turned off [23], a long pass filter $(>600 \mathrm{~nm})$ was placed in front of the PD. The OLED pixels were typically driven by $100 \mu \mathrm{s}$ $16-20 \mathrm{~V}$ pulses at $50 \mathrm{~Hz} ; 1200 \mathrm{PL}$ decay curves that followed the pulses were sampled and averaged to obtain $\tau$. A PMT was used to collect and amplify the PL signal.

\section{Results and discussion}

Results for three representative analytes of biomedical importance, i.e., glucose, cholesterol, and lactate, are shown below to demonstrate the operation and performance of the OLED and ZnO-based biosensing platform. Experiments were performed in open cells, in air, unless stated otherwise.

\subsection{Glucose sensor}

Composite films of PtOEP:PS: $\mathrm{TiO}_{2}$ with, on top, $\sim 96$ units of GOx sandwiched between two $\mathrm{ZnO}$ layers (a structure that was found to be the most usable) were first immersed in DI-water for $1 \mathrm{~min}$ to rinse off the unimmobilized enzyme. The enzymeleaching problem in the buffered water, which serves as the medium for the analytes, however, remained a challenge. Fig. 1 shows the results of repeated tests for a single sensor film. The enzyme activity was evaluated in terms of $\mathrm{O}_{2}$ consumption due to the enzymatic reaction of glucose (cholesterol and lactate are oxidized in similar reactions): 


$$
\text { Glucose }+\mathrm{O}_{2} \stackrel{\text { GOx }}{\longrightarrow} \mathrm{H}_{2} \mathrm{O}_{2}+\text { gluconic acid }
$$

Typical values of $\sim 30 \mu$ s and $\sim 100 \mu$ s represent $\tau$ of the PtOEP dye in the presence of $\sim 8.6$ ppm and 0 ppm DO, respectively, from which $K_{\mathrm{SV}}$ can be estimated to be $0.27 \mathrm{ppm}^{-1}$ or 8.97 $\mathrm{mM}^{-1}$. With the $\tau$ obtained after 1 min of the reaction and the known $K_{\mathrm{SV}}$, the DO level can be calculated based on Eq. (1). Assuming negligible in-diffusion of $\mathrm{O}_{2}$ into the solution at short reaction times, this DO level is subtracted from the initial value $(\sim 8.6 \mathrm{ppm}$ or $\sim 0.26$ $\mathrm{mM}$ in water at equilibrium with air at $\sim 23{ }^{\circ} \mathrm{C}$ ), yielding the amount of consumed DO.

As shown in Fig.1, after 8 identical runs, the consumed [DO] decreased by only $\sim 6 \%$. Enzyme leaching, which is believed to occur based on the usage of lower enzyme levels, as shown for LOx and ChOx, was slow, with the remaining immobilized level sufficient to sustain sensor operation. The glucose sensor film with the high enzyme load was thus adequate for at least 8 measurements, without introducing a significant error.

If the reaction takes place in a sealed cell, without $\mathrm{O}_{2}$ replenishing, [analyte] is related to $\tau$ by the modified SV relation [6]. This way, the highest detectable concentration is limited by the initial [DO] (this issue is eliminated through sample dilution). However, when the experiment is performed in cells exposed to air, DO consumption as well as $\mathrm{O}_{2}$ in-diffusion occur. This situation can extend the dynamic range, as shown in Fig. 2. Fig. 2a shows the leveling-off of $\tau$ as the glucose concentration increases; Fig. $2 b$ shows the linear calibration at the lower glucose concentrations. It shows that the upper limit of the dynamic range is $\sim 1.3 \mathrm{mM}$, which is 5 times higher than the initial DO level. We note that in experiments 
conducted in open cells, DO is monitored at a constant time following analyte-enzyme mixing. As such, linear calibration curves are obtained for $\tau$, rather than $1 / \tau$, vs [analyte].

In the dynamic process, competition between $\mathrm{O}_{2}$ in-diffusion and DO consumption determines [DO] at the time $\tau$ is measured. If the analyte and enzyme concentrations are large, the reaction becomes fast enough and potentially unaffected by $\mathrm{O}_{2}$ in-diffused when measured shortly after the analyte-enzyme mixing.

$\mathrm{O}_{2}$ replenishing was avoided in the sealed-cell tests, where the sensor film is at the bottom of the small glass container with a volume of $200 \mu \mathrm{L}$. The corresponding calibration curve is shown in Fig. 3, where the dynamic range extends to $\sim 0.3 \mathrm{mM}$. Note that the data used in Fig. 3 was obtained after 1 min of the oxidation reaction (Eq. 2), that is, before the reaction proceeded to completion. Thus, instead of a linear calibration of $1 / \tau$ vs [analyte $]_{\text {initial }}$, which is obtained when the reaction is completed, $\tau$ vs [analyte $]_{\text {initial }}$ was linear, as is the case for reactions monitored in open cells.

As seen in Eq. (2), glucose reacts with $\mathrm{O}_{2}$ at a 1:1 molar ratio. The initial reaction rate of glucose oxidation can be roughly estimated from the initial DO consumption rate. $\tau$ at 1 minute was used for obtaining the residual $[\mathrm{DO}]$; the $[\mathrm{DO}]$ prior to the reaction was, as mentioned, $8.6 \mathrm{ppm}(\sim 0.26 \mathrm{mM})$. Thus, the initial DO or analyte consumption rate could be obtained. A Lineweaver-Burk plot was constructed based on this initial glucose reaction rate and the corresponding glucose concentration. A good linear fit was obtained, as shown in Fig.4, from which the Michaelis constant $K_{\mathrm{m}} \sim 1.03 \mathrm{mM}$, was extracted. A $K_{\mathrm{m}}$ value of 2.19 $\mathrm{mM}$ was reported for a $\mathrm{ZnO}$-based electrochemical glucose sensor [10]. The relatively 
smaller $K_{\mathrm{m}}$ obtained for the OLED-based sensor using optical transduction indicates a high affinity between glucose and the GOx immobilized on the $\mathrm{ZnO}$ nanopartilces.

\subsection{Cholesterol sensor}

The enzyme load of ChOx was $\sim 2.3$ units, which is much lower than the GOx concentration used. The stability of the sensor film, with the identical sequential fabrication and testing, deteriorated significantly faster, impairing the applicability of the film with that ChOx level for repeated use. An alternative approach was therefore tested for the cholesterol sensor. For each [cholesterol] $]_{\text {initial }}$ measurement, a nominally identical disposable film was used, as shown in Fig. 5. As expected, higher concentrations result in longer $\tau$ values. As seen, during the first $\sim 3$ minutes, $\tau$ increases gradually due to a net decrease in [DO]; it levels off after $\sim 5$ minutes. This is followed by a decrease in $\tau$ (not shown), where the $\mathrm{O}_{2}$ indiffusion starts to affect $\tau$ significantly.

Fig.6. shows the linear calibration curve obtained by plotting $\tau$ at $5 \mathrm{~min}$, when the DO consumption and $\mathrm{O}_{2}$ replenishing are at a steady state. The dynamic range extends to $5.6 \mathrm{mM}$ or $217 \mathrm{mg} / \mathrm{dL}$, covering the range of normal cholesterol levels in human blood [24].

\subsection{Lactate sensor}

The enzyme load of SLOx was 4 units, which presented the same issue of enzyme leaching as for the cholesterol sensor. $100 \mathrm{mM}$ of lactate were used for repeated experiments to test the stability of the composite sensor film. After 4 identical runs, the enzyme activity was lowered by $\sim 16 \%$. Tests using disposable films for different lactate concentrations were therefore performed, as for the cholesterol sensor. A linear calibration curve was obtained, as shown in fig.7, with the dynamic range extending to $1 \mathrm{mM}$. 


\section{Concluding remarks}

To generate a biosensor in which major components are based on thin films, in addition to using a thin OLED excitation source, composite thin film probes for monitoring glucose, cholesterol, and lactate were evaluated. That is, in addition to embedding an $\mathrm{O}_{2}$ sensitive dye in a PS film, enzymes (that are necessary for the oxidation reactions of the above-mentioned analytes and thus for enabling analyte monitoring via residual DO level determination) were immobilized on $\mathrm{ZnO}$ nanoparticles. A capping, protecting $\mathrm{ZnO}$ layer was used to stabilize the sensor films, whose structure consisted of PtOEP: $\mathrm{TiO}_{2}$ : PS / ZnOenzyme / $\mathrm{ZnO}$. The PL decay time was used for monitoring the $\mathrm{DO}$ level, which is related to the analyte concentration. While the glucose sensing films were usable for repeated analyses, disposable films with lower enzyme concentrations were used for cholesterol and lactate monitoring.

Enzyme leaching remains the main culprit in generating an all thin-layer sensor film and thus a more compact sensor. This leaching may be due to weak adsorption of the first $\mathrm{ZnO}$ layer on the PtOEP: $\mathrm{TiO}_{2}$ : PS film. Another possibility, potentially contributing to enzyme leaching, is the porosity of the capping $\mathrm{ZnO}$ layer. A weaker nano $\mathrm{ZnO}$-enzyme stability in comparison to that under amperometric sensing conditions, as well as the nano $\mathrm{ZnO}$ attributes, may also affect sensor performance. A protective membrane, selectively permeable to water, which may alleviate such leaching, is currently being evaluated. And, to further reduce the overall sensor size, organic-based photodetectors are being evaluated for monitoring the PL. 


\section{Acknowledgements}

Ames Laboratory is operated by Iowa State University for the US Department of Energy (USDOE) under Contract No. DE-AC 02-07CH11358. This work was partially supported by the Director for Energy Research, Office of Basic Energy Sciences, USDOE.

\section{Figures}

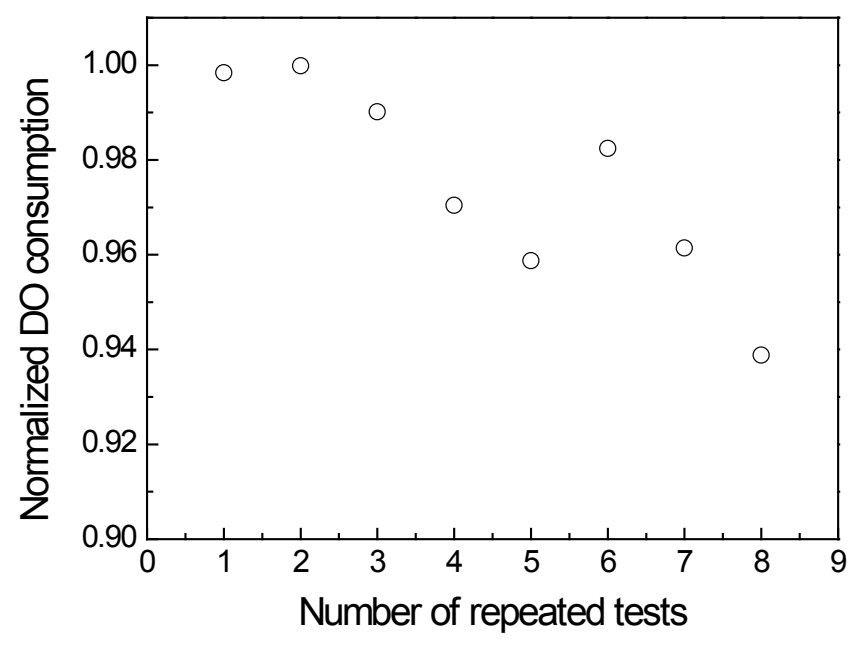

Fig. 1. Normalized consumed DO of a single sensor film following repeated tests using $40 \mu \mathrm{L}$ of $100 \mathrm{mM}$ glucose.
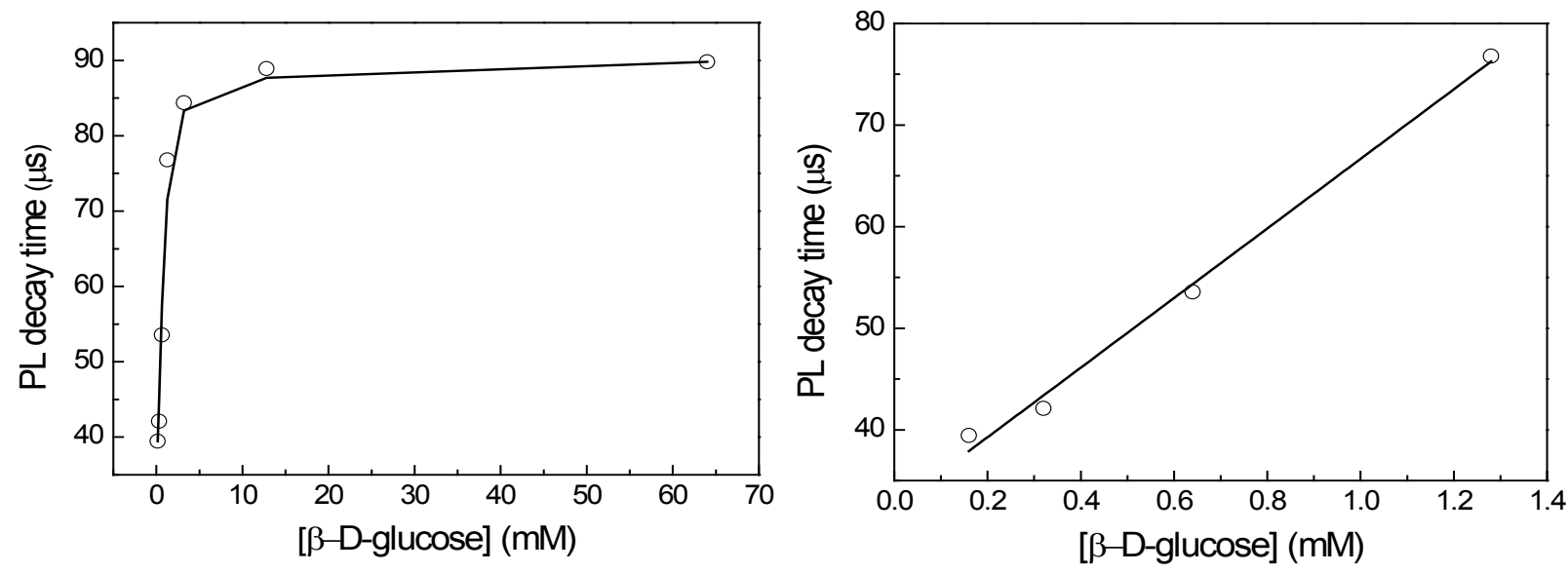

Fig. 2. Effect of glucose concentration on the PL decay time: left- the full range studied; right-linear calibration for low concentrations. 


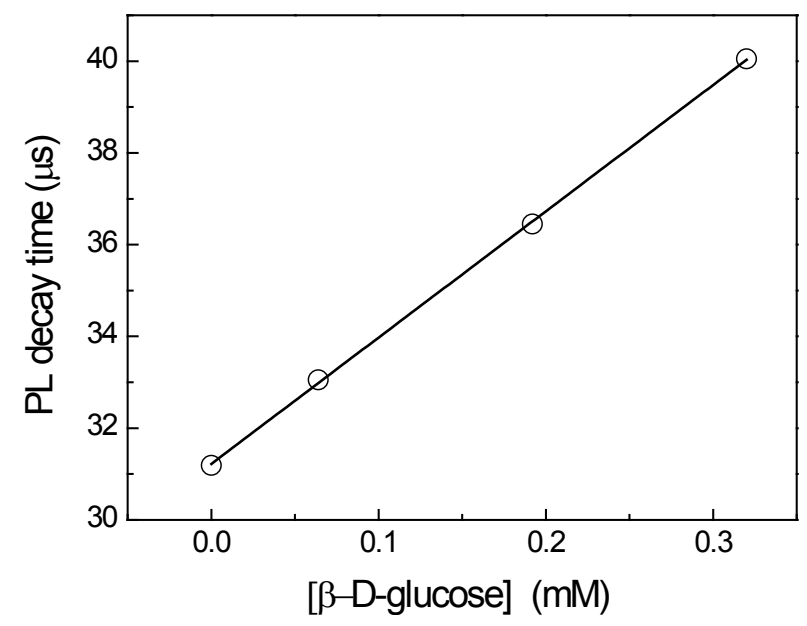

Fig.3. Effect of glucose concentration on the PL decay time after 1 minute of the reaction in a sealed cell.

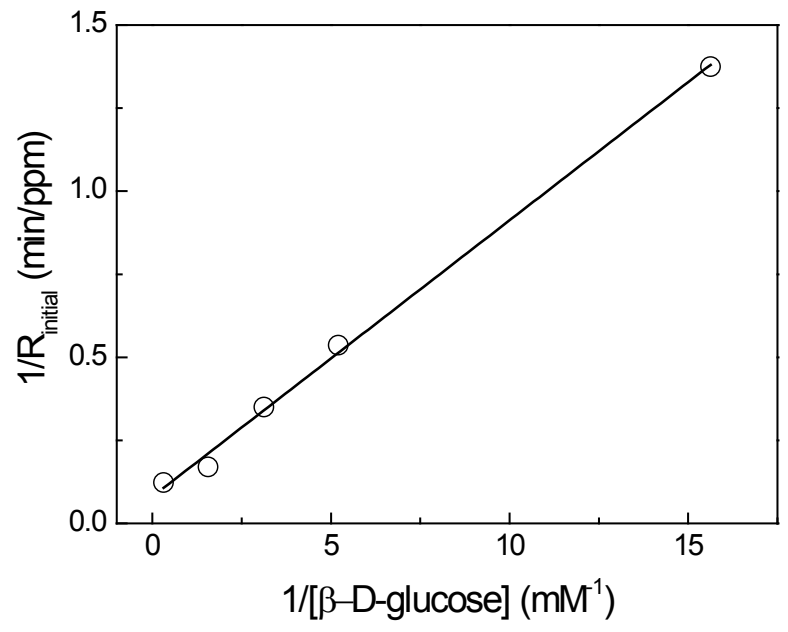

Fig.4. Lineweaver-Burk plot for sealed cell measurements of glucose. 


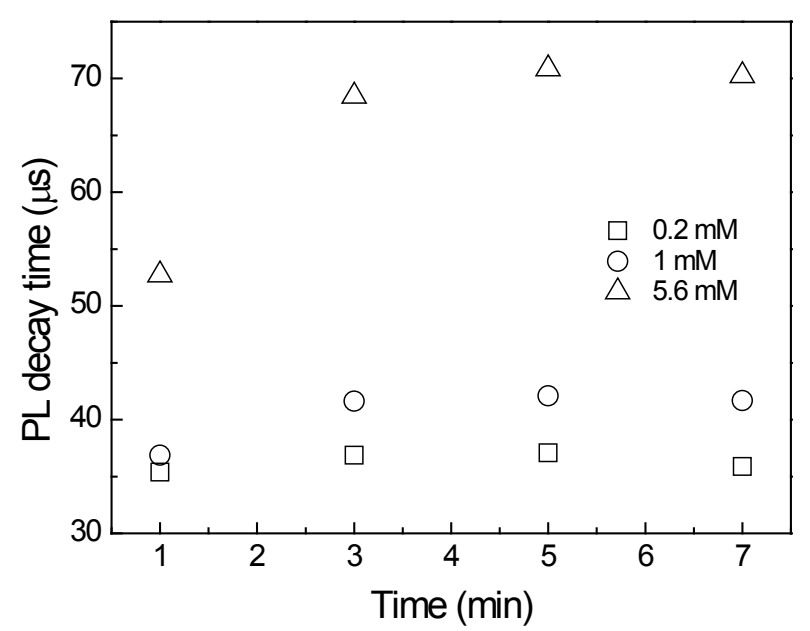

Fig. 5. Time-dependent PL decay time for different cholesterol concentrations.

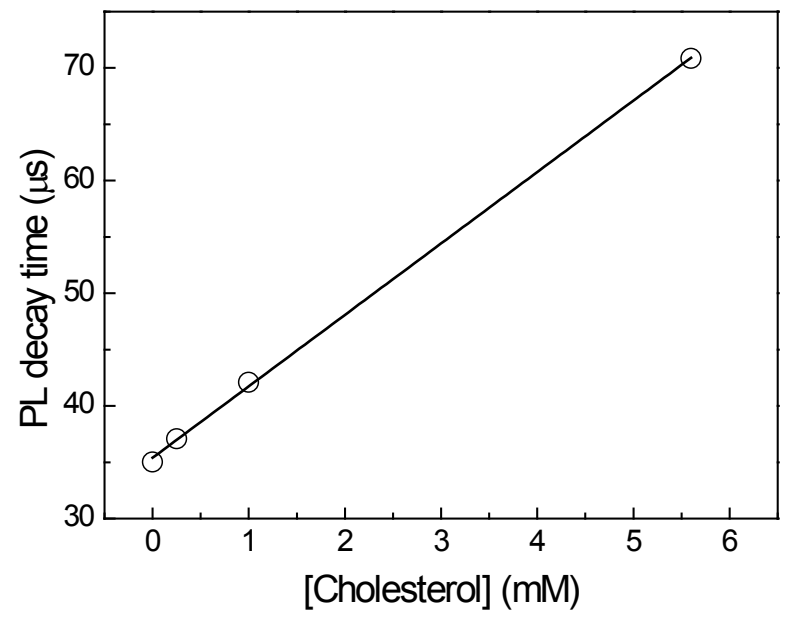

Fig. 6. Effect of cholesterol concentration on the PL decay time after 5 minutes. 


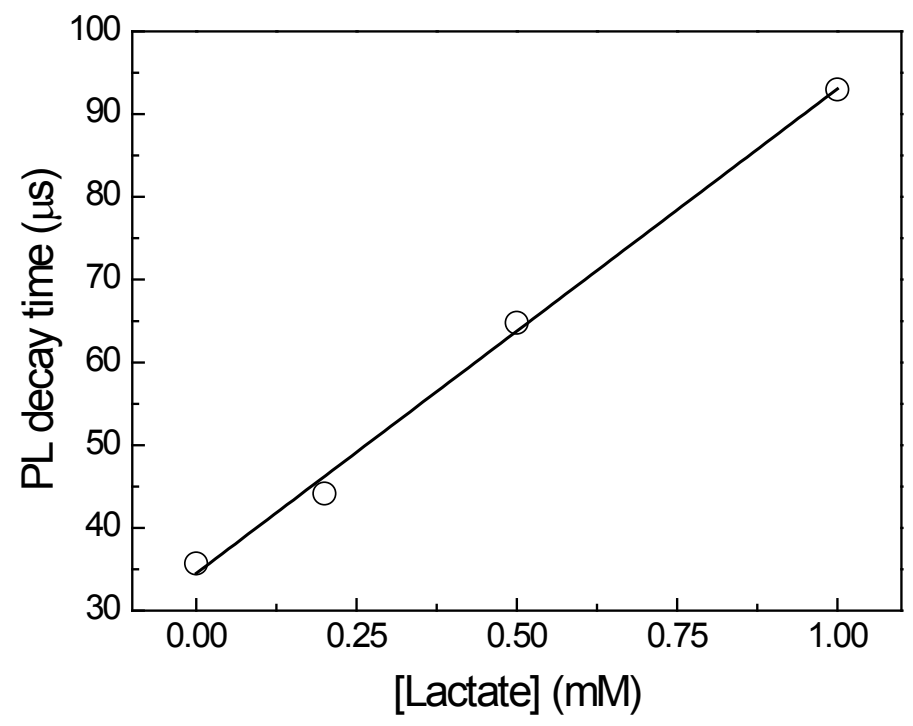

Fig. 7. Effect of lactate concentration on the PL decay time.

\section{References}

[1] B. Choudhury, R. Shinar, and J. Shinar, J. Appl. Phys., 96(5) (2004) 2949-2954.

[2] Z. Zhou, R. Shinar, B. Choudhury, L. Tabatabai, C. Liao, and J. Shinar, Proc. SPIE 5994 (2005) 5940E/1-5940E/9.

[3] R. Shinar, Z. Zhou, B. Choudhury, and J. Shinar, Anal. Chim. Acta, 568 (2006) 190-199.

[4] R. Shinar, D. Ghosh, B. Choudhury, M. Noack, V. L. Dalal, and J. Shinar, J. Non Cryst. Sol., 352 (2006) 1995-1998.

[5] Z. Zhou, R. Shinar, A. J. Allison, and J. Shinar, Adv. Func. Mater., 17 (2007) 3530-3537.

[6] Y. Cai, R. Shinar, Z. Zhou, and J. Shinar, Sensors and Actuators B, 134 (2008) 727-735.

[7] A. Wei, X. W. Sun, J. X. Wang, Y. Lei, X. P. Cai, M. Li, Z. L. Dong, and W. Huang, Appl. Phys. Lett., 89 (2006) 123902.

[8] Z. Dai, G. Shao, J. Hong, J. Bao, and J. Shen, Biosensors and Bioelectronics, 24 (2009) 1286-1291.

[9] J. Zang, C. M. Li, X. Cui, J. Wang, X. Sun, H. Dong, and C. Q. Sun, Electroanalysis, 19(9) (2007) 1008-1014.

[10] J. X. Wang, X. W. Sun, A. Wei, Y. Lei, X. P. Cai, C. M. Li, and Z. L. Dong, Appl. Phys. Lett., 88 (2006) 233106. 
[11] S. P. Singh, S. K. Arya, P. Pandey, B. D. Malhotra, S. Saha, K. Sreenjvas, and V. Gupta, Appl. Phys. Lett., 91 (2007) 063901.

[12] A. Umar, M. M. Rahman, M. Vaseem, and Y. B. Hahn, Electrochem. Comm., 11 (2009) 118-121.

[13] R. Khan, A. Kaushik, P. R. Solanki, A. A. Ansari, M. K. Pandey, and B. D. Malhotra, Anal. Chim. Acta, 616 (2008) 207-213.

[14] B. X. Gu, C. X. Xu, G. P. Zhu, S. Q. Liu, L. Y. Chen, M. L. Wang, and J. J. Zhu, J. Phys. Chem. B, 113 (2009) 6553-6557.

[15] B. X. Gu, C. X. Xu, G. P. Zhu, S. Q. Liu, L. Y. Chen, and X. S. Li, J. Phys. Chem. B, 113 (2009) 377-381.

[16] F. Zhang, X. Wang, S. Ai, Z. Sun, Q. Wan, Z. Zhu, Y. Xian, L. Jin, and K. Yamamoto, Anal. Chim. Acta, 519 (2004) 155-160.

[17] P. R. Solanki, A. Kaushik, A. A. Ansari, G. Sumana, and B. D. Malhotra, Appl. Phys. Lett., 93 (2008) 163903.

[18] E. Topoglidis, A. E. G. Cass, B. O’Regan, and J. R. Durrant, J. Electroanal. Chem., 517 (2001) 20-27.

[19] www.sigmaaldrich.com; www.aetltd.com

[20] M. Vafaee, and M. S. Ghamsari, Mater. Lett., 61 (2007) 3265-3268.

[21] M. S.Tokumoto, S. H. Pulcinelli, C. V. Santilli, and V. Briois, J. Phys. Chem. B, 107(2) (2003) 568-574.

[22] B. D. Yao, Y. F. Chan, and N. Wang, Appl. Phys. Lett., 81(4) (2002) 757-759.

[23] K. O. Cheon and J. Shinar, Phys. Rev. B, 69, (2004) 201306; Z. Gan, R. Liu, R. Shinar, and J. Shinar, submitted.

[24] www.lifeclinic.com 


\title{
Chapter 5. Multianalyte sensor array based on an organic light emitting
}

\section{diode platform}

A paper published in Sensors and Actuators B 134 (2008) 727-735

Y. Cai, R. Shinar, Z. Zhou, J. Shinar

\begin{abstract}
A compact photoluminescence (PL)-based sensor array, utilizing pulsed organic light emitting diode (OLED) pixels as the excitation sources, for sequential or simultaneous detection of multiple analytes in a single sample, is described. The utility and potential advantages of the structurally integrated OLED based platform for multianalyte detection are demonstrated for oxygen, glucose, lactate, and ethanol. The detection of glucose, lactate, and ethanol is based on monitoring the concentration of dissolved oxygen (DO) at the completion of the enzymatic oxidation reactions of these analytes in sealed cells. The monitoring in sealed cells and the ready access of the enzyme, when in solution, to the analyte enable a limit of detection of $\sim 0.02 \mathrm{mM}$, which is better than that obtained with enzymes embedded in sol-gel films. The DO concentration is determined via its effect on the PL decay time of the oxygen-sensitive dye Pt octaethylporphyrin embedded in a polystyrene film. A modified Stern-Volmer equation is derived to generate a linear calibration. The $2 \mathrm{~mm} \times 2 \mathrm{~mm}$ OLED pixels and the sensor films are fabricated on glass substrates that are attached back-to-back, generating a compact module devoid of any optical couplers. Two individually addressable OLED pixels are associated with the detection of each analyte. This configuration enables consecutive detection of all analytes within a few minutes utilizing a single photodetector
\end{abstract}


(PD). Simultaneous detection is achieved by using an array of small-size Si photodiode PDs compatible with the OLED pixel array. The OLED-based sensing array is unique in its ease of fabrication and integration with the sensing component, while its performance attributes are comparable to those obtained for detection of a single analyte using any excitation source.

\section{Introduction}

Multianalyte detection in a single sample using sensor arrays of various designs and sizes has been studied extensively with a range of transduction mechanisms, including electrochemical [1-3], piezoelectric [4], electrical resistance [5, 6], and optical [7-19]. Such studies are driven by the need for high throughput, inexpensive, and efficient analyses of complex samples in a broad range of applications, such as medical, biological (including biodefense), environmental, and industrial (e.g., the food and beverage industry). Sensor arrays are often fabricated by using photolithography and soft lithography $[2,3,6,20]$, inkjet-, screen-, and pin-printing $[13,14]$, and photodeposition $[7,8,20]$. These techniques frequently involve labor-intensive multistep fabrication, or require sophisticated image analysis and pattern-recognition codes.

In addition to monitoring multiple analytes, sensor arrays can be used to improve reliability in the monitoring of a single analyte through redundancy. That is, instead of using a single sensor to monitor the concentration of an analyte, an array of sensors is utilized, providing redundant information regarding the analyte concentration. Such electrochemical sensor arrays, employing redundant microelectrodes, were used for monitoring glucose, lactate, and pyruvate [2].

In this work we describe photoluminescence (PL)-based sensor arrays for simultaneous or sequential monitoring of multianalytes in a single sample. The excitation 
source is an array of individually addressable organic light emitting diode (OLED) pixels. It is compact and unique in its ease of fabrication and in the simplicity of the integration of the excitation source with the sensing component. Other light sources in PL-based sensors include lasers, lamps, and inorganic LEDs. However, such excitation sources are either bulky and/or costly, cannot be integrated with the other components due to size, geometrical, or operational constraints, or require intricate integration procedures for their incorporation in a structurally integrated, compact device [21].

OLED arrays can be based on single- or multi-color pixels fabricated in a combinatorial approach that results in adjacent OLED pixels that emit at wavelengths ranging from blue to red [22]. OLED pixels of nanometer size have been reported recently [23-25]; as such, they could be suitable for sensor microarrays for awide range of applications.

In addition to the small size of the OLED pixel excitation source, the advantage of the OLED-based sensing platform is also in its flexible design (OLEDs can be fabricated on plastic substrates), its compatibility with microfluidic architectures, and in its potential low cost, which will enable development of disposable sensors. Moreover, the thickness of the OLED excitation source is determined by that of the substrate, which will lead to fielddeployable, eventually badge-size sensors. Additional beneficial attributes of OLEDs in sensing applications are detailed below.

The OLEDs fabricated in this study were small-molecular OLEDs (SMOLEDs). They are easily fabricated using thermal evaporation in a low vacuum $\left(\sim 2 \times 10^{-6}\right.$ Torr $)$ evaporation chamber. Details on their fabrication and encapsulation, for enhanced long-term stability, are provided elsewhere [26, 27]. A typical SMOLED consists of a transparent conducting indium 
tin oxide (ITO)-coated glass or plastic substrate (the ITO is the anode), the organic layers that include hole transport layer(s) (HTLs), emitting layer(s), electron transport layer(s) (ETLs), and a metal, e.g., Al, cathode. The total thickness of the ITO, organic layers, and Al cathode is typically $<0.4 \mu \mathrm{m}$. Under forward bias, electrons are injected from the low-workfunction cathode into the ETL(s), e.g., tris(quinolinolate) $\mathrm{Al}\left(\mathrm{Alq}_{3}\right)$, which, for green OLEDs, is often also the emitting layer. Similarly, holes are injected from the high work function ITO into the HTL(s), often copper phthalocyanine (CuPc) followed by $N, N^{\prime}$-diphenyl- $N, N^{\prime}$-bis(1naphthylphenyl)-1,1_biphenyl-4,4'-diamine (NPD). Due to the applied bias, the electrons and holes drift towards each other, and recombine in the emitting layer. A certain fraction of the recombination events results in radiative excited states. These states provide the electroluminescence (EL) of the device.

The structurally integrated OLED/sensing component module is unique in its simplicity. The OLED and the sensing component are typically fabricated on separate transparent substrates (e.g., glass) that are attached back-to-back to form a module with a total thickness of $\sim 2 \mathrm{~mm}$ [27-29]. The photodetector (PD) can be located in front of the analyte cell ("front detection" geometry) or behind the OLED array, collecting the PL that passes through the gaps between the OLED pixels ("back detection" geometry) [29, 30]. The OLED excitation source can be operated by applying a DC bias (typically $\sim 5-20 \mathrm{~V}$ ) for measurements of analyte-induced PL intensity (I) changes. Alternatively, the OLEDs can be operated in a pulsed mode (typically at $\sim 10-20 \mathrm{~V}$ ); in $\mathrm{Alq}_{3}$ OLEDs, $\sim 99.99 \%$ of the EL signal decays within $<100 \mathrm{~ns}$, enabling the use of the OLEDs for monitoring the effect of analytes on the excited-state decay time $\tau$ of suitable luminophores. Operation in the $\tau$ mode is advantageous, as it eliminates the need for frequent sensor calibration. Frequent calibration 
and a reference sensor are needed when operating the sensors in the $I$ mode. This need is due to, e.g., changes in scattered light, and moderate changes in the sensor film or light source. The use of the OLED excitation source in conjunction with detection in the $\tau$ mode results in a compact module devoid of any optical couplers or filters.

OLEDs have dramatically improved over the past decade, and commercial products incorporating them are rapidly proliferating [31]. They are inherently advantageous as lowvoltage [32], miniaturizable [33], and flexible light sources [34, 35]. Electrophosphorescent green and blue OLEDs with an external quantum efficiency of $\sim 20 \%$ and $\sim 12 \%$, respectively, have been reported [35]. As mentioned, fabrication of nm-size OLED arrays has been demonstrated [23-25]; such arrays hold promise for OLED-based chemical and biological (micro)sensor arrays.

In this work, glucose, lactate, ethanol, and oxygen, which are of clinical, health, industrial (including the food and beverage industry), and environmental importance, are used to demonstrate the viability of the OLED-based multianalyte sensing platform. The detection is based on monitoring dissolved oxygen (DO) in sealed cells, utilizing an oxygensensitive dye, whose $I$ and $\tau$ decrease upon collisions with gas-phase $\mathrm{O} 2$ or DO [27, 29, 30, 36-40]. For monitoring glucose, ethanol, and lactate, in addition to the oxygen-sensitive dye, well known specific enzymes that oxidize these analytes, i.e., glucose oxidase (GOx), alcohol oxidase (AOx), and lactate oxidase (LOx), respectively, are used. Oxygen is consumed during the oxidation reactions, and in performing the reactions in a sealed cell, there is no replenishing of the DO. Consequently, the concentration of the DO at the completion of the oxidation reaction [DO]final, reflected by changes in $I$ and $\tau$ of the oxygen-sensitive dye PL, is determined by the analyte concentration. This approach, with the enzymes dissolved in 
solution, yields a lower (improved) limit of detection (LOD) than the one using immobilized enzymes [41], due to the accessibility of the enzymes and the ability to monitor the ppm levels of DO accurately.

Some of the above-mentioned analytes were previously monitored using sensor arrays. For example, a microelectrode array [1] was used to develop a multianalyte sensor for simultaneous detection of glucose, L-lactate, and uric acid. The fabrication of the array was based on CMOS technology and, as is often the case with electrochemical-based sensors, approaches to eliminate the effects of interferants were needed. An optical sensor for simultaneous detection of glucose and $\mathrm{O}_{2}$ was also reported [14]. The sensor was fabricated by pin-printing a Ru-based oxygen-sensitive dye and enzyme-doped xerogels, and using a laser or an LED as the excitation source. The LOD for glucose was $0.1-0.2 \mathrm{mM}$ with the poorer LOD obtained when using the LED. Intensity variability across the sensor array was observed when using the latter light source. Intensity variability issues are eliminated when operating the sensors in the $\tau$ mode, as done in the present work.

As in the case, mentioned above, of electrochemical sensor arrays employing redundant microelectrodes, an OLED-based array can also be used for redundancy or complementary analyte monitoring. For example, in monitoring $\mathrm{O}_{2}$ [27], arrays of similar sensor films, films prepared under different conditions containing a common analytesensitive dye, or films with different oxygen-sensitive dyes (e.g., Ru-based, Pt octaethylporphyrin (PtOEP), or the $\mathrm{Pd}$ analog PdOEP) can be used for simultaneous or sequential $\mathrm{O}_{2}$ monitoring by the different sensor films. This approach provides redundancy in the measurement, improving the accuracy, and enabling the use of different sensor films for monitoring $\mathrm{O}_{2}$ in different concentration regions using a single compact device. 
Simultaneous detection using several films can be achieved by using a small-size array of photodiodes, as described below.

In $\mathrm{O}_{2}$ sensors, the relation between the oxygen concentration $\left[\mathrm{O}_{2}\right], I$, and $\tau$ is ideally given by the Stern-Volmer (SV) equation:

$$
\frac{I_{0}}{I}=\frac{\tau_{o}}{\tau}=1+K_{S v}\left[O_{2}\right]
$$

where $I_{0}$ and $\tau_{0}$ are the unquenched values, $K_{\mathrm{SV}}=k \mathrm{q} . \tau_{0}$ is the Stern-Volmer constant, and $k_{\mathrm{q}}$ is the rate constant of the PL quenching reaction. However, deviations from that linear relation are quite common [27, 28, 36-39]. As shown below, the relation between $1 / \tau$ and glucose, lactate, or ethanol concentrations at a given initial DO concentration $[\mathrm{DO}]_{\text {initial }}$ can be expressed as a linear modified SVequation.

The OLED-based sensor array of this work demonstrates consecutive or simultaneous detection of glucose, lactate, and ethanol using a single PD (photomultiplier tube (PMT) or Si photodiode) or an assembled, small-size PD array of commercial Si photodiodes that are compatible with the OLED array design, respectively. As expected, comparable results were obtained using both approaches. As mentioned, the sensing film was based on PtOEP embedded in a polystyrene (PS) film that was structurally integrated with the OLED pixel array [27-29] to generate a compact device. The film served as the base of a cell that contained the analytes and the corresponding enzymes. The measurements were typically performed in sealed cells; in some cases, complex responses were obtained when the measurements were performed in cells open to air. The PDs were positioned in the backdetection geometry and monitored $\tau$, which eliminates the need for optical filters and frequent sensor calibration. The results are discussed in terms of the OLED-based sensor 
array attributes, including the array design, LOD, dynamic range, response time, and the reproducibility of the measurements. The potential for wide use of OLED-based sensors and their advantage for complex samples are also discussed.

\section{Experimental}

\subsection{Materials}

\subsubsection{Materials for OLED fabrication}

$20 \Omega / \square$ indium tin oxide (ITO)-coated glass was obtained from Applied Films Corp., $\alpha-\mathrm{NPD}$, coumarin (C545T), and $\mathrm{Alq}_{3}$ were obtained from H. W. Sands. CuPc and CsF were obtained from Sigma-Aldrich.

\subsubsection{Materials for sensing films}

PtOEP was obtained from H. W. Sands, PS, molecular weight 45,000, from SigmaAldrich, and toluene from Fisher Scientific. GOxfrom Aspergillus niger, AOxfromPichia pastoris, Lox from pediococcus species, and L-lactate were purchased from Sigma-Aldrich. Stabilized LOx from Aerococcus viridanswas obtained from Applied Enzyme Technology Inc. (Pontypool, UK). All chemicals were used as received.

\subsection{Instrumentation}

OLED arrays were fabricated by thermal vacuum evaporation of the organic layers in a home-built evaporation chamber (background pressure $\sim 2 \times 10^{-6}$ Torr) installed in an Arfilled glove box with typically $\sim 1 \mathrm{ppm}$ water and $\mathrm{O}_{2}$ levels. The OLEDs were driven by an AVTECH AV-1011B pulse-generator. The PD used for monitoring the sensor PL was a Hamamatsu R6060 PMT operated at 900V, or a 2 mm thick Hamamatsu S5107 $10 \mathrm{~mm} \times 10$ mm Si photodiode; an array of four Hamamatsu S5106 $5 \mathrm{~mm} \times 5 \mathrm{~mm}$ Si photodiodes, mounted on a circuit board containing an array of four preamplifier circuits, was used for the 
simultaneous detection of the four analytes. The PD was typically positioned in the "backdetection" geometry, collecting the PL passing through the gap between two OLED pixels that were used for the excitation [29, 30]. Gas-phase $\mathrm{O}_{2}$ and DO were monitored using flowing oxygen/argon mixtures, as previously described [27]. Mixing was achieved by means of mass flow controllers, where the flow rates of the oxygen and argon varied, while maintaining a constant total flow rate, thus generating varying oxygen partial pressures.

\subsection{Procedures}

\subsubsection{OLED fabrication}

OLED arrays were fabricated by thermal vacuum evaporation of organic layers on $\sim 100 \mathrm{~nm}$-thick ITO (the anode)-coated glass, which was treated as described previously [4244]. The organic layers consisted of a $5 \mathrm{~nm}$-thick $\mathrm{CuPc}$ hole injecting layer that is also believed to reduce the surface roughness of the ITO [45] and a 50 nm-thick NPD HTL. For

the green OLEDs, with peak emission at $\sim 530 \mathrm{~nm}$, the $\sim 40 \mathrm{~nm}$ thick emitting and ETL was either $\mathrm{Alq}_{3}$ or $\left(20 \mathrm{~nm} \mathrm{1wt. \%}\right.$ coumarin $(\mathrm{C} 545 \mathrm{~T})$-doped $\left.\mathrm{Alq}_{3}\right) /(25 \mathrm{~nm} \mathrm{Alq})$. An 8-10A CsF buffer layerwas deposited on the organic layers [46, 47], followed by the $\sim 150 \mathrm{~nm}$ thick Al cathode. The total thickness of the OLEDs, excluding the glass substrate, was thus $<0.4 \mu \mathrm{m}$. For measurements in the "back-detection" geometry, the OLEDs were prepared as an encapsulated matrix array of $\sim 2 \mathrm{~mm} \times 2 \mathrm{~mm}$ square pixels resulting from mutually perpendicular stripes of etched ITO and evaporated Al [29, 30]. Encapsulation was achieved by lining the edges of the glass substrate with high-vacuum Torr-Seal epoxy, and binding a top glass cover to the substrate. For $\tau$ measurements, the OLEDs were operated in a pulsed mode with a bias of 10-20 V, a pulse width of $100 \mu \mathrm{s}$, and a repetition rate of 50-200 Hz. 


\subsubsection{Sensing elements fabrication}

PtOEP-based sensing elements were prepared by dissolving $1-2 \mathrm{mg} / \mathrm{mL}$ of the dye and $40-100 \mathrm{mg} / \mathrm{mL}$ PS in toluene, and drop casting $20 \mu \mathrm{L}$ of the solution evenly onto cleaned glass slides. The resulting films, $\sim 7-9 \mathrm{~mm}$ in diameter and typically $\sim 8 \mu \mathrm{m}$ thick, were allowed to dry for $\sim 24 \mathrm{~h}$ in air in the dark at ambient temperature.

The oxidase enzymes were immobilized in thin sol-gel films based on known procedures [48]. The sol-gel films were dropcast or spin-coated over the PS:dye films [30]. Alternatively, the enzymes were dissolved in a $\mathrm{pH} 7.4$ buffer solution, in which their activity in repeated measurements in sealed cells was reproducible and their stability improved. Stability of enzymes is crucial for operation of biosensors, and their immobilization has been extensively studied [7, 48, 49]. However, prolonged use and storage of immobilized enzymes often remain a challenge $[7,47,48]$. Indeed, the oxidase-embedded films in this study, except for the films with GOx, were usable for a few measurements only, possibly due also to enzyme leaching. To evaluate the OLED-based platform for multiple analyte monitoring, we therefore typically used enzymes in buffered solution of 100-200 $\mu \mathrm{L}$ total volume, which were contained in glass wells. We note that the use of immobilized enzymes using improved approaches [49] is expected to similarly work with the OLED-based platform. The difference between an array with immobilized enzymes and that utilizing enzymes in solution in sealed cells is expected to be in the dynamic range and LOD, due to differences in the $\mathrm{O}_{2}$ level, its in-diffusion from the ambient, and enzyme accessibility.

The measurements in this work were performed at room temperature $\left(\sim 23{ }^{\circ} \mathrm{C}\right)$ or 37 ${ }^{\circ} \mathrm{C}$. The PS:dye sensor films were stable, revealing no significant changes in response to gasphase $\mathrm{O}_{2}$ or DO for a period exceeding 3 months. No dye leaching from the films was 
observed during this period, and in any case, operation in the $\tau$ mode is insensitive to small changes in the sensor film.

\subsubsection{Monitoring $\tau$}

The $\tau$ values were obtained by monitoring the PL decay curves following the OLED pulses. The decay curves were recorded at $20 \mathrm{~s}$ time intervals, starting $12 \mathrm{~s}$ after the addition of the analyte. It was possible to successfully fit the data to an exponential decay curve plus a constant, which reflects the background signal and/or offset level. The response time of the PMT is $\sim 5 \mathrm{~ns}$, and, as mentioned, the EL decays in $<100 \mathrm{~ns}$; both are much faster than the measured $\tau$ values. We note that the PL decay curves of a gas-phase $\mathrm{O}_{2}$ sensor often deviate from a simple exponential decay $[27,50-52]$, in particular at short $\tau$ values, corresponding to high oxygen concentrations ( $>\sim 20 \%$ ). In the current study, however, $\tau$ ranged from $\sim 28$ to 95 $\mu$ s with the shortest $\tau$ corresponding to a DO level in equilibrium with air (i.e., in the absence of an analyte). Based on repeated experiments, the experimental error in $\tau$ was found to be smaller than $5 \%$.

\subsection{4. $\mathrm{O}_{2}$ level measurements}

Measurements of gas-phase oxygen using the integrated $\mathrm{Alq}_{3}$ OLEDs/PtOEP-based filmwere performed in a flowcell with flowing oxygen/argon mixtures. Mixing was achieved by means of mass flow controllers, as described above.

Measurements of DO in water were performed in a sealed cell. The gas-phase $\mathrm{O}_{2}$ was allowed to equilibrate with the solution following initial bubbling of $\mathrm{O} 2 / \mathrm{Ar}$ mixtures through the solution and continued flow at the gas-phase/liquid interface during the measurement [27]. This approach resulted in reproducible and reliable results, as the value of $\tau$ measured when 
the solution was in equilibrium with ambient air was within experimental error of the value measured when the solution was exposed to $21 \% \mathrm{O}_{2}$ in $\mathrm{Ar}$. The DO level in water at $23{ }^{\circ} \mathrm{C}$, in equilibrium with ambient air at $1 \mathrm{~atm}$, is about $8.5 \mathrm{ppm}$.

We note that monitoring [DO] indicated that at the ethanol concentrations used in this study there was no observable increased miscibility of $\mathrm{O}_{2}$.

\subsubsection{Glucose, lactate, and ethanol monitoring}

Single and multiple analytes in buffered solutions were monitored via their effect on $\tau$ following their enzymatic oxidation reactions. In the sequential measurements, groups of two OLED pixels were lit consecutively, using the PMT for monitoring the PL. In the simultaneous monitoring mode, all OLED pixels were lit simultaneously, and the PL of each analyte was monitored by its associated $5 \mathrm{~mm} \times 5 \mathrm{~mm}$ Si photodiode. A Labview program was created to enable simultaneous monitoring via separate channels.

\section{Results and discussion}

Structurally integrated OLED/sensing film modules for monitoring oxygen and glucose were reported earlier $[27,29,30]$. In brief, for $\mathrm{O}_{2}$ detection, PtOEP or PdOEP were embedded in a PS film, and $\mathrm{Alq}_{3}: \mathrm{C} 545 \mathrm{~T} / \mathrm{Alq}_{3}$ or rubrene-doped $\mathrm{Alq}_{3}$ OLEDs, respectively, were used as the excitation source to monitor the effect of the analyte concentration on $\tau$. SVcalibration curves for gas-phase and DO were obtained [27]. For glucose detection, a solgel film with embedded GOx was drop-cast or spin-coated on top of the PtOEP:PS film [30].

$I$ and $\tau$ were measured as a function of glucose concentrations. For both OLED-based sensors, the dynamic range and LOD were comparable or improved in comparison to previously reported sensors based on a variety of other excitation sources [27, 30]. The dynamic range of the glucose sensor covered the physiological range. 
For ethanol and lactate detection, embedding the enzymes (including the stabilized ones) in a similar sol-gel or polymeric film appeared to adversely affect the long-term enzyme activity, rendering the sensors usable for a few measurements only. Such sensors can therefore be re-used with disposable enzyme-containing films (in contrast, the OLEDs and the PS:PtOEP films are stable for many months). To demonstrate the multianalyte sensor, we therefore used a PS:PtOEP film, but kept the enzyme in a solution to which the analytes (single analyte or mixtures)were added. This approach resulted in reproducible results.

The analytes were monitored by determining $[\mathrm{DO}]_{\text {final }}$ (in sealed reaction cells) at the completion of the analytes' oxidation reactions. That is, the DO that was not consumed in the oxidation reactions was monitored.

In the presence of GOx and $\mathrm{O}_{2}$, glucose is oxidized to yield gluconic acid as oxygen is reduced to $\mathrm{H}_{2} \mathrm{O}_{2}$ :

$$
\text { Glucose }+\mathrm{O}_{2} \stackrel{\mathrm{GO}_{\mathrm{x}}}{\longrightarrow} \mathrm{H}_{2} \mathrm{O}_{2}+\text { Gluconic acid }
$$

LOx and AOx similarly oxidize lactate and ethanol, respectively. Therefore, in sealed containers, where there is no supply of DO beyond the initial concentration, the change in [DO] is proportional to the change in the analyte concentration [analyte]. If [analyte $]_{\text {initial }}$ $\leq[\mathrm{DO}]_{\text {initial }}$, and assuming that the analyte conversion into products is complete, which was confirmed by the present results, $[\mathrm{DO}]_{\text {final }}$ is given by

$$
[\mathrm{DO}]_{\text {final }}=[\mathrm{DO}]_{\text {initial }}-[\text { analyte }]_{\text {initial }}
$$

Within the assumption stated above, this equation is valid for sealed wells, as mentioned, and for containers open to air, where the oxidation reaction is much faster than the in-diffusion rate of gas-phase oxygen. In the latter case (see below), [DO] should be 
monitored shortly (depending on the enzyme concentration) after the addition of the analyte. The SV equation becomes, accordingly:

$$
\frac{I_{0}}{I}=\frac{\tau_{0}}{\tau}=1+K_{s v} \times\left\{[D O]_{\text {initial }}-[\text { analyte }]_{\text {initial }}\right\}
$$

Thus, a plot of $1 / \tau$ vs. the initial analyte concentration in the test solution will ideally be linear up to $[\mathrm{DO}]_{\text {initial }}=[\text { analyte }]_{\text {initial, }}$, with the slope yielding the value of the rate constant of the PtOEP PL quenching reaction by DO, $k_{\mathrm{q}}$. This rate constant depends on the oxygen solubility in the film and its diffusion coefficient, and is therefore film and temperaturedependent.

If the above-mentioned assumptions are valid for reactions such as shown by Eq. (2), under comparable experimental conditions, and given sensor film and analyte concentration (i.e., given $\left.[\mathrm{DO}]_{\text {final }}\right), \tau$ values for glucose, lactate, or ethanol are expected to be identical, since they depend only on $[\mathrm{DO}]_{\text {final }}, k_{\mathrm{q}}$, and $\tau_{0}$.

The foregoing closed-cell sensor limits the apparent dynamic range at $\sim 23{ }^{\circ} \mathrm{C}$ to [analyte $_{\text {initial }} \sim 0.25 \mathrm{mM}$, i.e., $8 \mathrm{ppm} \mathrm{DO}$, which approaches the $[\mathrm{DO}]_{\text {initial }}$ level of $8.5 \mathrm{wt}$. ppm in equilibrium with air at that temperature. However, this limited dynamic range corresponds only to the analyte concentration in the final test solution, where it is diluted, i.e., added to the enzyme-containing buffer solution. Thus, the actual dynamic range is wide, and can cover the concentration range of medical/industrial interest. Moreover, as an example, it has been shown that there is a linear relation (albeit patient-dependent) between blood and saliva glucose levels, with typical levels of $\sim 0.02$ to $\sim 0.2 \mathrm{mM}$ in the latter. As such, the approach described here, with its LOD of $\sim 0.02 \mathrm{mM}$, can be developed as a possible alternative for blood testing [53]. 


\subsection{Sensor array design and approaches to multianalyte testing}

In the sequential operation mode, a single sample containing a mixture of the analytes was placed above the different OLED pixels/sensing films; the OLED pixels were energized in succession to monitor each analyte (in the presence of the corresponding enzyme) separately. This operation mode required $\sim 5 \mathrm{~min}$ to determine the level of all four analytes. We note that the response time of the oxygen sensor is $\sim 1-2 \mathrm{~s}$ [27], however, in all the measurements of this study, the slower enzymatic reactions, which depend on the enzyme concentration, determined the overall monitoring time, which was $\sim 0.5-5 \mathrm{~min}$. In the simultaneous detection mode, the four $5 \mathrm{~mm} \times 5 \mathrm{~mm}$ Si photodiodes were assembled in an array that was designed to be compatible with the OLED pixel array. The analyte mixtures were analyzed simultaneously on the OLED array, with the output of each PD corresponding to a different analyte. We note that various Si-based PD arrays with rectangular $1-4 \mathrm{~mm}^{2}$ elements are available commercially (from, e.g., Hamamatsu) and can be used in conjunction with OLED arrays for a larger number of analytes. Additionally, work is in progress to develop $\mu \mathrm{m}$-thick thin film PDs, such as those based on amorphous or nanocrystalline Si, that are fabricated together with OLED pixels on a common substrate, to generate a compact, fully integrated PL-based sensor array for multianalytes [28]. This type of advanced integration is unique to the OLED-based platform, and can potentially lead to miniaturized (micro) sensor arrays.

Before proceeding to the multianalyte sensors,we first describe the measurements on a single analyte. 


\subsection{Lactate, ethanol and glucose sensors}

As seen in Fig. 1a, when lactate was monitored at $\sim 37{ }^{\circ} \mathrm{C}$ in a container open to air, $\tau$ increased initially to some maximal value $\tau_{\max }$ due to the decreasing DO level in the presence of the analyte + enzyme. As expected, $\tau_{\max }$ decreased with decreasing analyte concentrations. However, following the initial increase to $\tau_{\max }$, which was fast due to the relatively large (10 units $/ \mathrm{mL}$ ) enzyme concentration, $\tau$ decreased, indicating in-diffusion of $\mathrm{O}_{2}$ from the gasphase into the solution. The slow decrease of $\tau$ with $t$, in particular at the higher lactate concentrations, is probably due to slow permeability of DO into the sensor film. As seen in the figure, for the sensor and enzyme concentration used in this example, $\tau$ should be measured within $\sim 10-20$ s of exposure to the analyte.

As expected, the rate of increase of $\tau$ to $\tau_{\max }$ depended on the enzyme concentration; for lower enzyme concentrations, the oxidation reaction was significantly slower (e.g., $\tau_{\max }$ was obtained after $\sim 2.5 \mathrm{~min}$ at $23^{\circ} \mathrm{C}$ when using 1.5 units $/ \mathrm{mL}$ enzyme, as shown in Fig. $1 \mathrm{~b}$ ). The measured $\tau_{\max }$ then reflects a quasi-steady-state DO, wherein the reduction in the [DO] due to the oxidation reaction is counter-balanced by the in-diffusion of oxygen.

The calibration curve of $1 \tau_{\max }$ vs. [lactate] $]_{\text {initial}}$, corresponding to the oxidation of lactate (seen in Fig. 1a), was in agreement with Eq. (4). In that case, the dynamic range extended from a solution with no lactate, where $[\mathrm{DO}]_{\text {initial }} \sim 7 \mathrm{ppm}$ (the equilibrium value in air at $37{ }^{\circ} \mathrm{C}$ ) to a solution practically depleted of DO in the vicinity of the film, i.e., $\sim 0.22$ $\mathrm{mM}$ analyte. Over this range, $\tau_{\max }$ typically increased from $\sim 25$ to $\sim 100 \mu \mathrm{s}$, depending on the film. 
The oxidation reactions were relatively slow. For example, 100 units/mL GOx, 7.5 units/mL stabilized LOx, and 125 units/mL AOx required 100, 30, and 120 s, respectively, for completion of the oxidation reaction. Despite these relatively long times, a calibration curve for a given film could be obtained at shorter times by monitoring $\tau$ after a constant reaction time for different analyte concentrations, and, as observed, by monitoring $\tau$ as a function of time and plotting the initial rate of change in $\tau$ vs. the analyte concentration. Such a calibration curve is shown in Fig. 2 for lactate and 1 unit/mL enzyme. In this example, the initial rate of change in $\tau$ was obtained from the initial $4-5$ data points (up to $\sim 83 \mathrm{~s}$ ), which represented the linear part of $\tau$ vs. the reaction time. The dynamic range using this approach was about $0.1-0.8 \mathrm{mM}$.We note that this initial rate of change in $\tau$ is not directly related to the initial reaction rate. As seen in Eq. (4), $1 / \tau$ is linear in $[\mathrm{DO}]_{\text {final }}$ in the $0-0.25 \mathrm{mM}$ range. However, plots of the initial rate of change in $1 / \tau$ vs. the initial analyte (glucose, lactate, or ethanol) concentration did not always yield usable calibration curves. Additionally, we could not obtain a conclusive Michaelis-Menten constant from such curves.

Measurements in sealed containers did not exhibit the decrease in $\tau$ shown in Fig. 1a; in contrast, $\tau$ remained largely constant at the completion of the reaction. Using the modified SV plot (Eq. (4)), i.e., plotting $1 / \tau$ at the completion of the oxidation reactions vs. [analyte $]_{\text {initial, }}$ resulted in, as expected, linear calibrations for analyte concentrations up to $\sim 0.25 \mathrm{mM}$ at $23{ }^{\circ} \mathrm{C}$. Fig. 3 shows such calibration curves for glucose and lactate; the experiments were conducted at $23{ }^{\circ} \mathrm{C}$ in sealed containers on different films. Monitoring ethanol resulted in comparable results. 
Fig. 3 demonstrates that the LOD is $\sim 0.02 \mathrm{mM}$ for lactate and glucose. This improved LOD was attained, since [DO], which can be easily measured at low ppm levels, was monitored in 100-200 $\mu \mathrm{L}$ sealed cells with relatively high levels of accessible enzymes. In contrast, when using smaller amounts of analyte solutions on an oxidase-doped sol-gel film [30], the faster in-diffusion of $\mathrm{O}_{2}$, and possibly limited enzyme accessibility, deteriorate the LOD, increasing it to $\sim 0.1 \mathrm{mM}$ [41]. As shownbelow, the calibration curve shown in Fig. 3 was successfully adopted for monitoring analyte mixtures with a similar low LOD.

\subsection{Multianalyte sensing}

\subsubsection{Sequential monitoring}

Fig. 4 shows the platform for such a multianalyte sensor, where six pairs of green $\mathrm{Alq}_{3}$ OLED pixels are lit simultaneously; the OLED pixels are defined by the overlap between the mutually perpendicular ITO and Al stripes. The leftmost and rightmost pairs are bare OLED pixels. A PS:PtOEP film was drop cast on the 2nd, 3rd, 4th, and 5th pairs; the 2nd pair was used for monitoring oxygen in the absence of the other analytes. A GOx-doped sol-gel film was dropcast from a $7.5 \mathrm{mg} / \mathrm{mL} \mathrm{GOx}+$ sol-gel solution on the 3rd pair, while 3 units/mL AOx and 1.5 units/mL LOxwere contained each in a separate glass well, whose base was the PS:PtOEP film above pairs \#4 and \#5, respectively. The orange appearance of pairs 2-6 is due to the superposition of the green Alq ${ }_{3}$ OLED EL and the red PtOEP PL. In this example analyte solutions were placed consecutively over the sensing elements and the appropriate pixels were addressed to detect each of the analytes. The measurement was performed in air using a PMT. The figure shows also the decay curves and the exponential fit. Since the glucose, alcohol, and lactate levels were high, the expected value of $\tau$ was $\sim 95-100$ 
$\mu$ s. Yet the observed values were $80-87 \mu$ s due to a non-negligible DO level resulting from in-diffusion of some oxygen from the air.

In sealed containers, calibration curves for the three analytes were obtained using their mixtures at different concentrations. Fig. 5 shows $1 / \tau$ vs. [analyte] $]_{\text {initial }}$ for lactate, ethanol, and glucose for the same PS:PtOEP film. As seen, the data for all three analytes can be presented by a single calibration line independent of the analyte, whether glucose, lactate, or ethanol. This is expected, since under these experimental conditions the similar oxidation reactions of all three analytes proceeded to completion, and [DO $]_{\text {final, }}$, which is related to the analyte concentration (Eq. (3)), was monitored. No interference between the analytes was observed and the reaction for a given analyte occurred only in the presence of the specific corresponding enzyme. In the presence of that specific oxidase, $\tau$ was determined (to within less than $1.5 \%$ ) only by the level of the analyte that corresponds to that specific oxidase. For example, in the presence of LOx, $\tau$ for 0.1 and $0.2 \mathrm{mMlactate}$ varied from 45.3 to $46.1 \mu \mathrm{s}$ and from 68.0 to $69.1 \mu \mathrm{s}$, respectively, in the presence of any combination of 0.15 or $0.35 \mathrm{mM}$ ethanol and 0.15 or $0.35 \mathrm{mM}$ glucose. In the presence of GOx, $\tau$ for $0.35 \mathrm{mM}$ glucose varied in the range $79.6-81.7 \mu \mathrm{s}$ in the presence of 0.1 or $0.2 \mathrm{mM}$ lactate and 0.15 or $0.35 \mathrm{mM}$ ethanol.

\subsubsection{Simultaneous monitoring}

Fig. 6(a) shows one basic design of the OLED pixel array for the simultaneous multianalyte monitoring platform; as in the design shown in Fig. 4, the OLED pixels are defined by the overlap between the mutually perpendicular ITO and Al stripes. The pixel size is typically $2 \mathrm{~mm} \times 2 \mathrm{~mm} ; 0.3 \mathrm{~mm} \times 0.3 \mathrm{~mm}$ pixels were also tested and found similarly 
adequate for use as the excitation source for sensing applications. We note that there is no cross talk between the OLED pixels. As in the sequential operation mode (Fig. 4), two pixels were used for each of the four analytes.

Fig. 6(b) shows an array of four wells mounted on the OLED array, with the four 5 $\mathrm{mm} \times 5 \mathrm{~mm}$ Si photodiodes, mentioned in Section 3.1 above, positioned underneath the OLED array.

Fig. 7 shows the calibration curves obtained for single analytes together with data points obtained for analyte mixtures measured later to check the validity of the approach for (unknown) mixtures. In all cases the OLED array pixels were lit simultaneously and $[\mathrm{DO}]_{\text {final }}$ or each analyte concentration was measured with its specific photodiode; the Labview software handled all the data concurrently. The measurement time, determined by the rate of the oxidation reactions, was $\sim 1-2 \min$. As seen, similar to the sequential monitoring, the compact OLED-platform is suitable for simultaneous monitoring of multiple analytes in a mixture. As was the case for the consecutive measurements, a single line fits the results for all analytes up to concentrations approaching [DO]initial, and the analysis results of mixtures fit the linear calibration curves of the single analytes very well; the LOD was $\sim 0.02 \mathrm{mM}$, as in monitoring of the single analytes. Hence, the compact OLED platform is suitable for simultaneous monitoring of multiple analytes in a mixture.

For all the sensor films used in this study $k_{q}$ for DO in the water and PS film at $\sim 23{ }^{\circ} \mathrm{C}$ was in the range of $(9 \pm 2) \times 10^{7} \mathrm{M}^{-1} \mathrm{~s}^{-1}$. As expected, this value is similar to the value we found in evaluating the OLED-based platform for monitoring DO [27]. 


\section{Summary and concluding remarks}

A compact, structurally integrated, photoluminescent OLED based multianalyte sensor for monitoring gas-phase oxygen and DO, glucose, alcohol, and lactate was demonstrated. The advantage of this OLED-based platform is in its flexible design and small size, individually addressable pixel configuration, unique ease of fabrication and integration of the OLED with the sensing component, compatibility with microfluidic structures, as well as the possibility to fabricate OLEDs on plastic substrates, and eventually, develop disposable badge-size sensor modules. All of the sensing elements included a PS:PtOEP film; the PL of the PtOEP is collisionally quenched by $\mathrm{O}_{2}$. The addition of glucose-, alcohol-, and lactate-oxidase catalyzes the oxidation of the corresponding analytes, resulting in depletion of the oxygen in the solution and consequently, an increase in the PL intensity $I$ and decay time $\tau$. The sensors in thiswork monitored the analytes' concentrations by determining the latter. In contrast to the $I$ mode, the $\tau$ mode eliminates the need for frequent sensor calibration. The multianalyte sensor was operated in either a sequential mode by individually addressing the OLED pixels that excite a given sensing element, or in a simultaneous mode, where all OLED pixels were energized simultaneously and the analytes were monitored using an array of photodiodes compatible with the OLED pixel array, with each photodiode

used for a specific sensor in the array. The sensors' performance was evaluated in terms of the dynamic range, the LOD, the analytes' interference with each other, the response time, and the reproducibility of the measurements. A modified Stern-Volmer calibration curve, linear with [analyte] $]_{\text {initial }}$ for concentrations up to [DO]initial, was used successfully for monitoring reproducibly mixtures of glucose, lactate, and ethanol in sealed containers with no interference between the analytes. Since $[\mathrm{DO}]_{\text {initial }} \sim 8.5 \mathrm{ppm}$ under ambient conditions, the 
apparent dynamic range was limited to [analyte $]_{\text {initial }} \sim 0.25 \mathrm{mM}$ in the test solution. However, since the analyte sample is diluted prior to the measurement this limit is eliminated straightforwardly. Moreover, conducting the experiments with solutions in seals cells with easily accessible enzymes enabled attaining a low LOD value of $\sim 0.02 \mathrm{mM}$, which is significantly better than the $\sim 0.1 \mathrm{mM}$ obtained by PL-based methods using immobilized enzymes. The measurement time, which reflects the oxidation reactions time under the specific experimental conditions, was typically $\sim 1-2 \mathrm{~min}$ in the simultaneous measurement and a total of $\sim 5$ min for all of the analytes in the sequential measurement. The response time of the oxygen sensor is $\sim 1-2 \mathrm{~s}$.

Eight pixels of a single-color OLED array were utilized in the present demonstration of the viability of the structurally integrated platform for multianalyte detection in mixtures. However, OLED arrays typically contain many more pixels, so that several sensors can be employed for each analyte. The use of such redundant sensors should enable monitoring the analytes' concentration with increased accuracy.

\section{Acknowledgments}

We thank Chengliang Qian for technical assistance. Ames Laboratory is operated by Iowa State University (ISU) for the United States Department of Energy (USDOE) under Contract DE-AC 02- 07CH11358. This work was partially supported by the Director for Energy Research, Office of Basic Energy Sciences, USDOE, the ISU Institute for Physical Research and Technology, the National Aeronautics and Space Administration, and the National Science Foundation.

\section{References}

[1] H. Frebel, G.-C. Chemnitius, K. Cammann, R. Kakerow, M. Rospert, W. Mokwa, 
Sens. Actuators B, 43 (1997) 87-93.

[2] A.F. Revzin, K. Sirkar, A. Simonian, M.V. Pishko, Sens. Actuators B, 81 (2002) 359-368.

[3] M.S. Wilson, W. Nie, Anal. Chem., 78 (2006) 2507-2513.

[4] E.T. Zellers, M. Han, Anal. Chem., 68 (1996) 2409-2418.

[5] M.S. Freund, N.S. Lewis, Proc. Natl. Acad. Sci. U.S.A., 92 (1995) 2652-2656.

[6] A. Carbonaro, L. Sohn, Lab Chip, 5 (2005) 1155-1160.

[7] L. Li, D.R.Walt, Anal. Chem., 67 (1995) 3746-3752.

[8] T.A. Dickinson, J. White, J.S. Kauer, D.R.Walt, Nature, 382 (1996) 697-700.

[9] D.R. Walt, T. Dickinson, J. White, J. Kauer, S. Johnson, H. Engelhardt, J. Sutter, P. Jurs, Biosens. Bioelectron., 13 (1998) 697-699.

[10] K.L. Michael, L.C. Taylor, S.L. Schultz, D.R.Walt, Anal. Chem., 70 (1998) 1242-1248.

[11] C.A. Rowe, L.M. Tender, M.J. Feldstein, J.P. Golden, S.B. Scruggs, B.D. MacCraith, J.J. Cras, F.S. Liegler, Anal. Chem., 71 (1999) 3846-3852.

[12] M.D. Marazuela, M.C. Moreno-Bondi, Chem., 372 (2002) 664-682.

[13] E.J. Cho, F.V. Bright, Anal. Chem., 74 (2002) 1462-1466.

[14] E.J. Cho, Z. Tao, E.C. Tehan, F.V. Bright, Anal. Chem., 74 (2002) 6177-6184.

[15] V.K. Yadavalli, W.-G. Koh, G.J. Lazur, M.V. Pishko, Sens. Actuators B, 97 (2004) 290297.

[16] E.R. Goldman, A.R. Clapp, G.P. Anderson, H.T. Uyeda, J.M. Mauro, I.L. Mendintz, H. Mattoussi, Anal. Chem., 76 (2004) 684-688.

[17] N.M. Grubor, R. Shinar, R. Jankowiak, M.D. Porter, G.J. Small, Biosens. Bioelectron., 19 (2004) 547-556.

[18] M. Vollprecht, F. Dieterle, S. Busche, G. Gauglitz, K.-J. Eichhorn, B. Voit, Anal. Chem., 77 (2005) 5542-5550.

[19] J. Liu, J.H. Lee, Y. Lu, Anal. Chem., 79 (2007) 4120-4125.

[20] G. McGall, J. Labadie, P. Brock, G.Wallraff, T. Nguyen,W. Hinsberg, Proc. Natl. Acad. 
Sci. U.S.A., 93 (1996) 13555-13560.

[21] E.J. Cho, F.V. Bright, Anal. Chem., 73 (2001) 3289.

[22] K.O. Cheon, J. Shinar, Appl. Phys. Lett., 83 (2003) 2073-2075.

[23] J.G.C. Veinot, H. Yan, S.M. Smith, J. Cui, Q. Huang, T.J. Marks, Nano Lett., 2 (2002) $333-335$.

[24] F.A. Boroumand, P.W. Fry, D.G. Lidzey, Nano Lett., 5 (2005) 67-71.

[25] C.H. Yamamoto, J. Wilkinson, J.P. Long, K. Bussman, J.A. Christodoulides, Z.H. Kafafi, Nano Lett., 5 (2005) 2485-2488.

[26] J. Shinar, V. Savvate'ev, in: J. Shinar (Ed.), Springer Verlag, NY, 2003, pp. 1-41 (Chapter 1).

[27] R. Shinar, Z. Zhou, B. Choudhury, J. Shinar, Anal. Chim. Acta, 568 (2006) 190-199.

[28] R. Shinar, D. Ghosh, B. Choudhury, M. Noack, V.L. Dalal, J. Shinar, J. Non Cryst. Solids, 352 (2006) 1995-1998.

[29] R. Shinar, C. Qian, Y. Cai, Z. Zhou, B. Choudhury, J. Shinar, Smart Medical and Biomedical Sensor Technology III, Proc. SPIE, 6007 (2005) 600710-1.

[30] B. Choudhury, R. Shinar, J. Shinar, J. Appl. Phys., 96 (2004) 2949-2954.

[31] http://optics.org/articles/news/11/9/16?alert=1; http://optics.org/articles/news/12/6/6/1.

[32] R.H. Friend, R.W. Gymer, A.B. Holmes, J.H. Burroughes, R.N. Marks, C. Taliani, D.D.C. Bradley, D.A. Dos Santos, J.L. Bredas, M. Logdlund, W.R. Salaneck, Nature, 397 (1999) 121-128.

[33] http://www.emagin.com.

[34] G. Gustafsson, Y. Cao, G.M. Treacy, F. Klavetter, N. Colaneri, A.J. Heeger, Nature, 357 (1992) 477-479.

[35] www.universaldisplay.com.

[36] C. McDonagh, B.D. MacCraith, A.K. McEvoy, Anal. Chem., 70 (1998) 45-50.

[37] D. Garcia-Fresnadillo, M.D. Marazuela, M.C. Moreno-Bondi, G. Orellana, Langmuir, 15 (1999) 6451-6459. 
[38] R.N. Gillanders, M.C. Tedford, P.J. Crilly, R.T. Bailey, Anal. Chim. Acta, 502 (2004) 16.

[39] C.S. Burke, O. McGaughey, J.-M. Sabattie, H. Barry, A.K. Mcevoy, C. McDonagh, B.D. MacCraith, Analyst, 130 (2005) 41-45.

[40] A.J. Palma, J. Lo`ıpez-Gonza`1lez, L.J. Asensio, M.D. Ferna`ındez-Ramos, L. Fermı^1n Capita`n-Vallvey, Anal. Chem., 79 (2007) 3173-3179.

[41] O.S. Wolfbeis, M. Schaeferling, A. Duerkop, Microchim. Acta, 143 (2003) 221-227.

[42] V. Savvate'ev, J.H. Friedl, L. Zou, J. Shinar, K. Christensen, W. Oldham, L.J. Rothberg, Z. Chen-Esterlit, R. Kopelman, Appl. Phys. Lett., 76 (2000) 1501-1503.

[43] K.O. Cheon, J. Shinar, Appl. Phys. Lett., 81 (2002) 1738-1740.

[44] G. Li, J. Shinar, Appl. Phys. Lett., 83 (2003) 5359-5361.

[45] L. Zou, V. Savvate'ev, J. Booher, C.-H. Kim, J. Shinar, Appl. Phys. Lett., 7 (2001) $2282-2284$.

[46] L.S. Hung, C.W. Tang, M.G. Mason, Appl. Phys. Lett., 70 (1997) 152-154.

[47] S.E. Shaheen, G.E. Jabbour, M.M. Morell, Y. Kawabe, B. Kippelen, N. Peyghambarian, M.-F. Nabor, R. Schlaf, E.A. Mash, N.R. Armstrong, J. Appl. Phys., 84 (1998) 23242327.

[48] O.S. Wolfbeis, I. Oehme, N. Papkovskaya, I. Klimant, Biosens. Bioelectron., 15 (2000) 69-76.

[49] P. Pandey, S.P. Singh, S.K. Arya, V. Gupta,M. Datta, S. Singh, B.D. Malhotra, Langmuir, 23 (2007) 333-3337 (and references therein).

[50] A. Mills, Sens. Actuators B, 51 (1998) 69-76.

[51] P. Roche, R. Al-Jowder, R. Naravanaswamy, J. Young, P. Scully, Anal. Bioanal. Chem., 386 (2006) 1245-1257.

[52] Z. Zhou, R. Shinar, A.J. Allison, J. Shinar, Adv. Funct. Mater., 17 (2007) 3530-3537. 


\section{Figures}
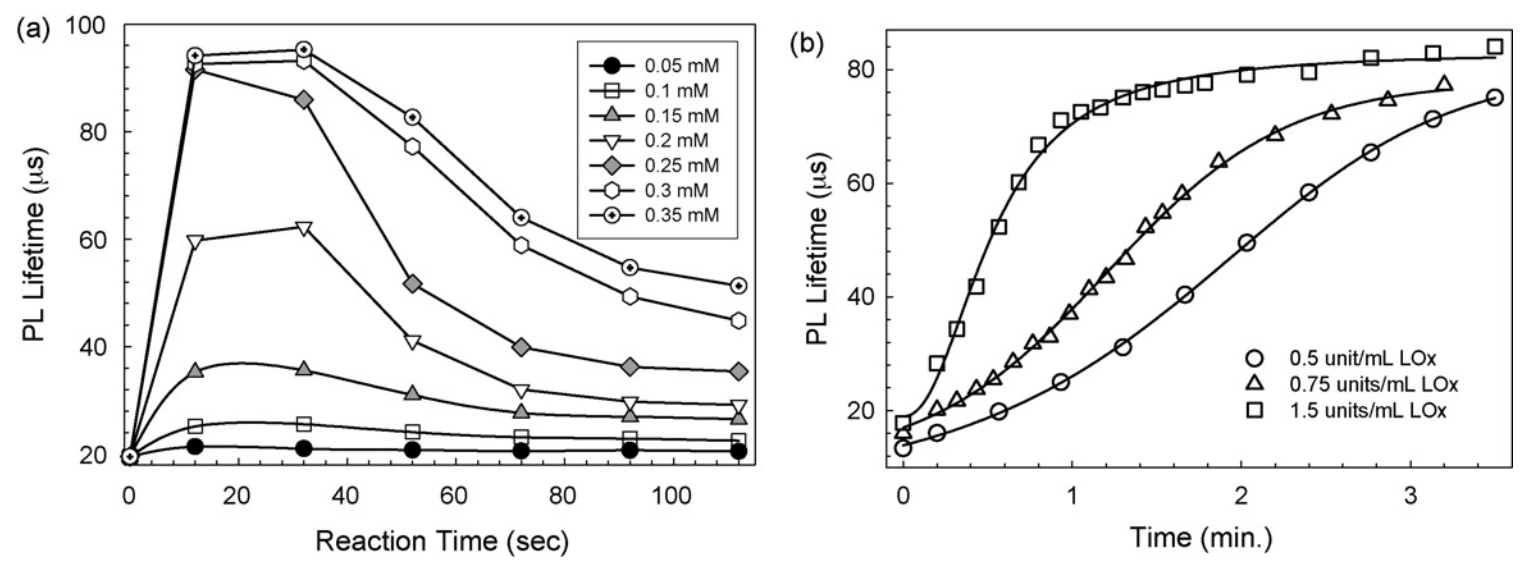

Fig. 1. (a) $\tau$ vs. the reaction time upon addition of lactate to an LOx solution, for various lactate concentrations (see inset). The LOx concentration was 10 units $/ \mathrm{mL}$. The measurement was performed at $37{ }^{\circ} \mathrm{C}$ in a cell open to air. (b) $\tau$ vs. the reaction time for $0.3 \mathrm{mM}$ lactate and LOx levels of 0.5 units $/ \mathrm{mL}$ (circles), 0.75 units $/ \mathrm{mL}$ (triangles), and 1.5 units $/ \mathrm{mL}$ (squares). The measurements were performed in an open cell at $23{ }^{\circ} \mathrm{C}$.

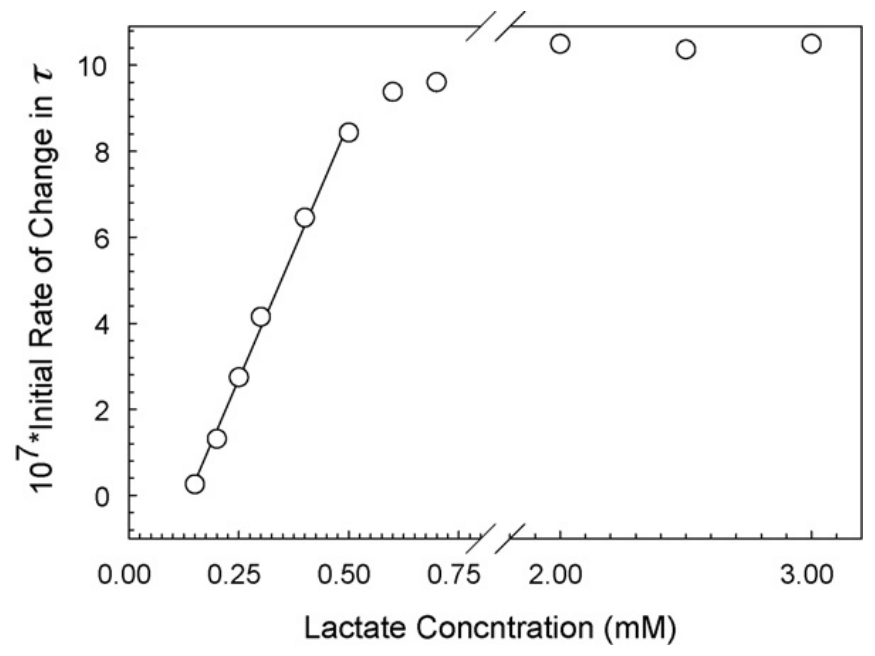

Fig. 2. The initial rate of change in $\tau$ vs. the lactate concentration. The enzyme concentration was 1 unit/mL. The measurement was performed at $37^{\circ} \mathrm{C}$ in a sealed cell. 


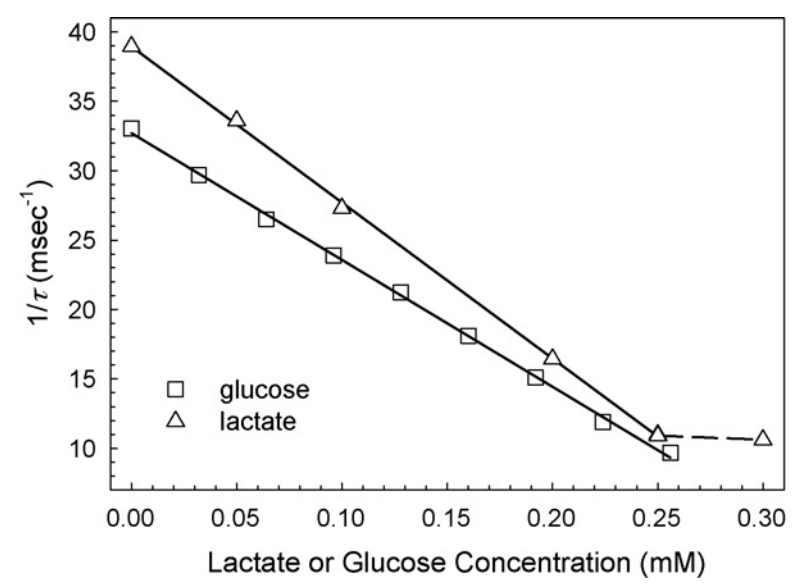

Fig. 3. $1 / \tau$ vs. the lactate or glucose concentrations. The measurements were performed using two different films at $\sim 23{ }^{\circ} \mathrm{C}$ in sealed containers.

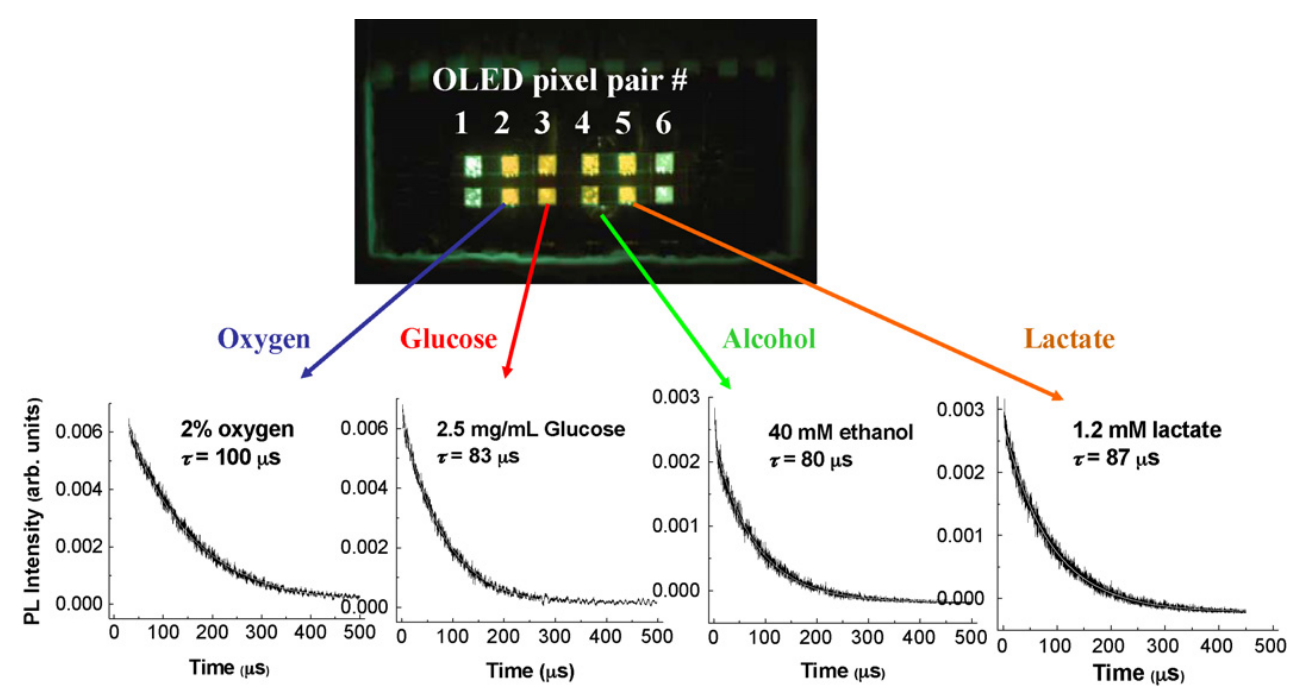

Fig. 4. Structurally integrated OLED-based photoluminescent multianalyte sensor for sequential monitoring of oxygen, glucose, alcohol, and lactate. All of the sensing elements were based on a PS:PtOEP film, positioned above OLED pixel pairs 2-5. The orange-yellow appearance of these pixels is due to the superposition of the green emission from the $\mathrm{Alq}_{3^{-}}$ based OLED and the red emission from the PS:PtOEP film. The analyteswere monitored via the PL lifetime $\tau$ of the PtOEP. The figure shows the intensity as a function of time (black lines) and the exponential fit (white lines). Measurements were conducted in air at $<23{ }^{\circ} \mathrm{C}$. (For interpretation of the references to color in this figure legend, the reader is referred to the web version of the article.) 


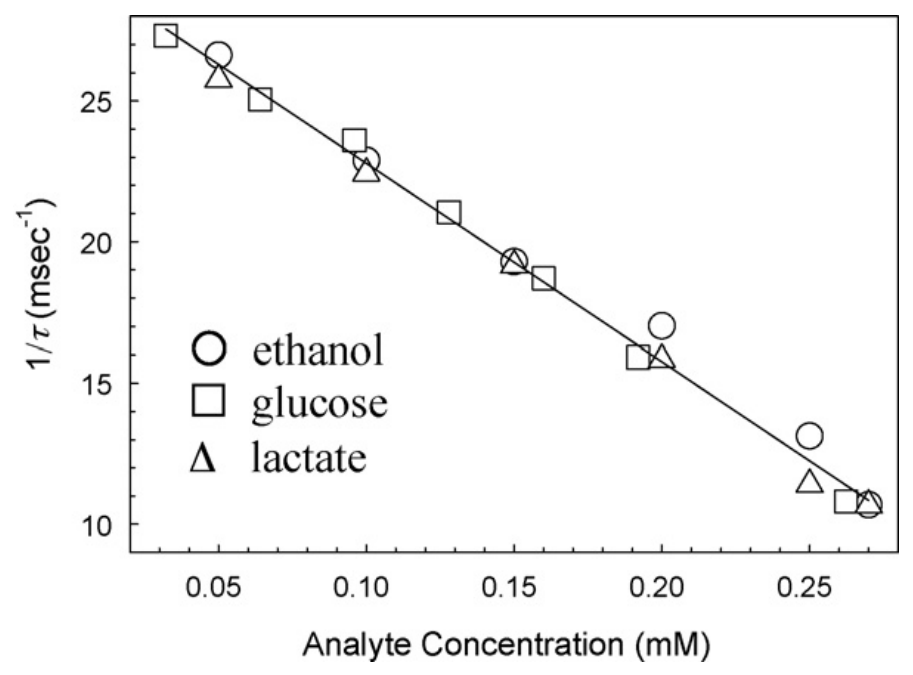

Fig. 5. $1 / \tau$ vs. the analyte concentrations for multianalyte measurements performed consecutively at $22{ }^{\circ} \mathrm{C}$ in a sealed container. A single photodetector (PMT)was used.

(a)

OLED pixel

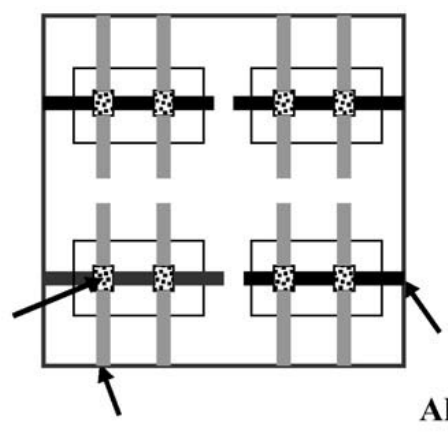

Al cathode (b)

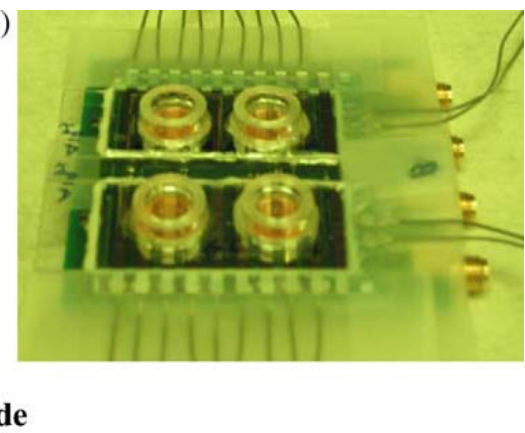

ITO anode

Fig. 6. (a) Schematic of the OLED array designed for simultaneous monitoring of four analytes. The vertical lines are the ITO anode stripes, the horizontal lines are the Al cathode stripes. The (square) OLED pixels are defined by the overlap between the ITO and the Al stripes. (b) 2" $\times 2$ " structurally integrated OLED-based photoluminescent multianalyte sensor for simultaneous monitoring of oxygen, glucose, alcohol, and lactate. The PS:PtOEP film is located at the bottom of each of the wells, an OLED array is located under each PS:PtOEP film, a $5 \mathrm{~mm} \times 5 \mathrm{~mm}$ Si photodiode is located under each OLED array, and a preamplifier circuit is located under each photodiode. Each well contains a buffer solution with no oxidase, GOx, LOx, or AOx to which the sample is added. 


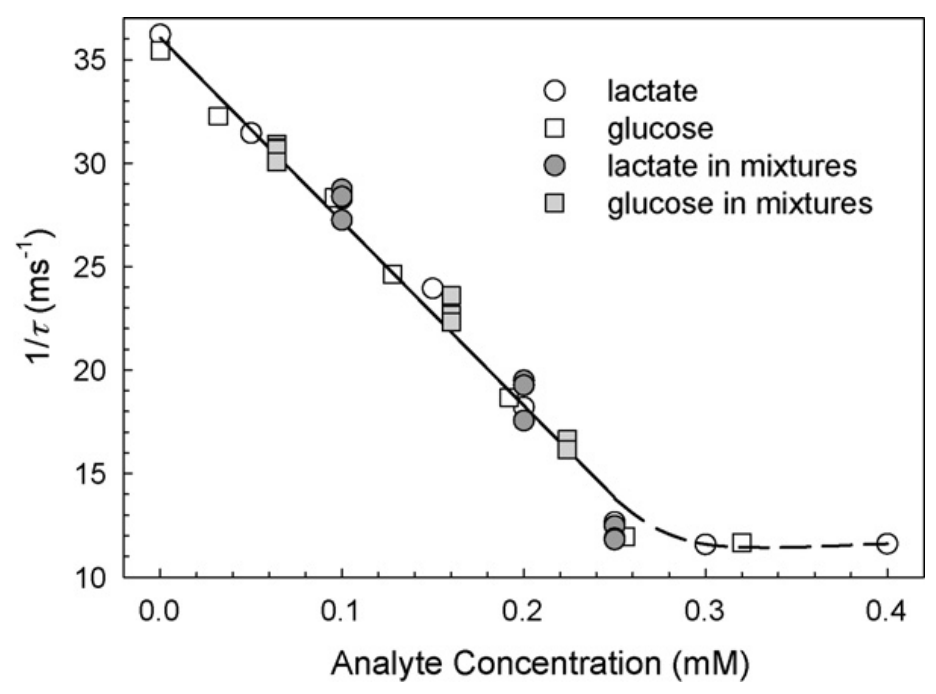

Fig. 7. $1 / \tau$ vs. the analyte concentrations for multianalyte measurements performed simultaneously at $\sim 22^{\circ} \mathrm{C}$ in a sealed container. The open symbols indicate data points used to generate the calibration curves; the filled points are different mixtures of all analytes used for testing the analysis of mixtures. Detection was performed by use of an array of $5 \mathrm{~mm} \times 5 \mathrm{~mm}$ Si photodiodes. 


\title{
Chapter 6. Data analysis and aging in phosphorescent oxygen-based
}

\author{
sensors \\ A paper published in Sensors and Actuators B 146 (2010) 14-22 \\ Y. Cai, A. Smith, J. Shinar, R. Shinar
}

\begin{abstract}
The stretched exponential analysis of the photoluminescence (PL) decay curves of the oxygen-sensitive dye Pt octaethylporphyrin (PtOEP) embedded in a polystyrene (PS) film and used in gas-phase oxygen, dissolved oxygen (DO), glucose, and lactate sensors is discussed. Light emitting diodes (LEDs) and organic LEDs (OLEDs) served as the pulsed excitation sources for the PL. Typically, the stretched exponential analysis resulted in excellent fits of the oxygen-quenched PL decay curves, superior to the single exponential analysis, in particular at the higher oxygen levels. While some previous studies of gas-phase oxygen sensors analyzed the decay curves with a single value of the stretching factor $\beta$, and other studies used the product of a single exponential and a stretched exponential with a fixed $\beta$, in this study only the stretched exponential term was used with $\beta$ as a variable. As a result, $\beta$ was found to decrease with increasing $\mathrm{O}_{2}$ concentration $\left(\left[\mathrm{O}_{2}\right]\right)$, from $\beta=1$, i.e., a simple exponential decay, at gas-phase $\left[\mathrm{O}_{2}\right]=0$ and $[\mathrm{DO}]=0$. The effect of doping the PtOEP:PS films with $360 \mathrm{~nm}$ titania particles (which enhance the PL) on the data analysis was also examined. In general, the $\mathrm{TiO}_{2}$ increased $\tau$ and $\beta$. The results indicate that a distribution of $\mathrm{O}_{2}$ : dye collision rates, induced by the microheterogeneity of the sensor films, is responsible for the nonexponential decay kinetics. The $\left[\mathrm{O}_{2}\right]$-dependent $\beta$ is possibly associated with
\end{abstract}


shallow multiple quencher trapping sites in the PS matrix that affect the frequency of dye: $\mathrm{O}_{2}$ collisions. Additionally, the long-term stability, data analysis, and detection sensitivity of the DO sensor during and following one-year aging, with the sensing film constantly immersed in water, are described. The findings impact commercial PL-based DO sensors.

\section{Introduction}

Photoluminescence (PL)-based oxygen sensors have been studied extensively [1-17], and such devices, monitoring gas-phase and dissolved oxygen (DO), are available commercially. The PL-based sensors are advantageous over the electrochemical sensors due to attributes such as improved stability, lower maintenance, and less-frequent calibration. Moreover, issues common to electrochemical sensors, including oxygen consumption and electrode poisoning, are eliminated.

The PL-based oxygen sensors typically utilize an oxygen-sensitive dye embedded in a thin polymeric or sol-gel film. When the excited dye collides with oxygen molecules its PL is quenched with a dose-dependent decrease in the PL intensity $I$ and decay time $\tau$. Calibration lines and the oxygen level can be obtained using the Stern-Volmer (SV) equation

$$
I_{0} / I=\tau_{0} / \tau=1+K_{\mathrm{SV}}\left[\mathrm{O}_{2}\right]
$$

where $I_{0}$ and $\tau_{0}$ are the unquenched values and $K_{\mathrm{SV}}$ is the film and temperature-dependent SV constant.

When using thin-film-based sensors, the ideal behavior described by Eq. 1 is often not obeyed $[8,12-17]$. This situation is usually due to microheterogeneity of the matrix and consequently, to luminophore molecules in sites that are not equally accessible to the quencher. Thus, several approaches have been suggested to modify Eq. 1. As an example, Eq. 
1 was modified to include a sum of two or multiple exponential terms representing luminophore/site combinations with specific $K_{\mathrm{SV}}$ and fractional contributions that depend on the local environment of the luminophore [1-3]. Indeed, the use of a two-site model, with two discrete sets of quenching parameters, resulted in improved fits of $I_{0} / I$ vs $\left[\mathrm{O}_{2}\right]$ plots for Ru dyes in some polymer and sol-gel derived matrices $[1-3,11]$. Similar to the PL intensity, the PL time-resolved intensity decay kinetics was described as a sum of individual singleexponential components with characteristic $\tau$ values and pre-exponential amplitudes [11]. In another work [4], it was shown that a fit of the decay kinetics to a sum of exponentials for a $\mathrm{Ru}$ dye in various polymers results in an unreasonable dependence of the pre-exponential factors on the oxygen pressure. A distribution of relaxation rates, based on the interaction of the dye with its heterogeneous environment, was therefore proposed. This model required a smaller number of fitting parameters in comparison to the multi-exponential model. In another model [7], which resulted in a comparable decay function and was also developed to include the influence of the microenvironment on the PL decay time, it was assumed that the PL quenching due to luminophore-polymer matrix interactions depends on the distance between the luminophore and the nearest interacting polymer site. As such, the quenching rate of a given excited molecule is the sum of its distance-dependent interactions with a number of quenching sites. According to the authors [7], for the examples they provided, this model is physically and practically advantageous over the multi-exponential and ratedistribution models. It was simplified by Bossi et al. [9] who showed that the nonexponential PL decay of two Ru dyes embedded in polydimethylsiloxane (PDMS) was accurately described by the function

$$
I(t)=I_{0} \exp \left(-B t^{0.5}\right),
$$


i.e., a special case of the stretched exponential behavior $[4,7,9,13-15,18-21]$ described below, where the stretching factor $\beta=0.5$. Bossi et al. [9] suggested that this behavior was due to saturation of $\left[\mathrm{O}_{2}\right]$ in the PDMS film, i.e., $\left[\mathrm{O}_{2}\right]$ in the film was sublinear in the $\mathrm{O}_{2}$ partial pressure of the surrounding gas. In all these examples, the single exponential analysis of the PL decay curves was inadequate even in the absence of the oxygen quencher. The stretched exponential behavior

$$
I(t)=I_{0} \exp \left[\left(-t / \tau_{k w w}\right)^{\beta}\right]
$$

is often used to describe dispersive processes in polymers. $\tau_{k w w}$ is a characteristic value ( $k w w$ refers to Kohlrausch-Williams-Watts, who applied the stretched exponential function to relaxation and scattering processes in disordered systems [18]). The stretched exponential behavior is a result of the microheterogeneity in disordered solid matrices and that disorder is quantified by the deviation of the parameter $\beta$ from unity [19]. The ensemble average $<\tau>$ and the square root of the variance $\sigma^{1 / 2}$ of the decay times distribution are determined from $\tau_{k w w}$ and $\beta[13]:$

$$
\begin{aligned}
& <\tau>=\left(\tau_{k w w} / \beta\right) \Gamma\left(\beta^{-1}\right) \\
& \sigma^{1 / 2}=\left\langle\tau>w=<\tau>\left[\beta \Gamma\left(2 \beta^{-1}\right)-\Gamma^{2}\left(\beta^{1}\right)\right]^{1 / 2} / \Gamma\left(\beta^{1}\right)\right.
\end{aligned}
$$

where

$$
\Gamma(x)=\int_{0}^{\infty} z^{x-1} \exp (-z) d z
$$

is the Gamma function and $w$ is the relative (dimensionless) distribution width [13]. 
Besides the analysis by Bossi et al., where $\beta=0.5$, an analysis using the product of a simple exponential and a stretched exponential successfully described the PL decay of Pd tetraphenyl-porphyrin (PdTPP) encapsulated in polymethylmethacrylate (PMMA) [13]. In that analysis, $\beta$ varied from 0.73 to 0.79 over the first $10 \mathrm{~h}$ of measurements on their films.

In the present work, we describe the use of the stretched exponential analysis to obtain the (least squares) values of $\beta,\langle\tau\rangle$, and $\sigma^{1 / 2}$ for the PL of Pt octaethylporphyrin (PtOEP) embedded in polystyrene (PS) (PtOEP:PS) that is quenched by either gas-phase $\mathrm{O}_{2}$ or DO. We also include the analysis of $360 \mathrm{~nm} \mathrm{TiO}_{2}$ particle-doped PtEOP:PS films, since these particles enhance the PL up to 10 fold [17]. That analysis indeed shows that the $\mathrm{TiO}_{2}$ increases $\left\langle\tau>\right.$ and $\beta$, consistent with additional trapping of the $\mathrm{O}_{2}$ at sites induced by the $\mathrm{TiO}_{2}$. This analysis, in which the fitted parameters (besides the amplitude $I_{0}$ and the background level) are $\tau_{k w w}$ and $\beta$, resulted in better fits of the oxygen-quenched PL decay curves following pulsed excitation. More importantly, it provided insight into the nature of the microheterogeneity of the environment in which the PL quenching $\mathrm{O}_{2}$ diffuses. Specifically, it was found to be consistent with a scenario in which the $\mathrm{O}_{2}$ diffuses through a multiple-trapping-site system. The analysis was used for gas-phase oxygen and DO sensors as well as for glucose and lactate sensors. The latter are based on monitoring oxygen following the oxidation reaction of glucose (lactate) in the presence of glucose (lactate) oxidase and oxygen [22-25]. Oxygen is consumed during the oxidation reactions, resulting in enhanced $I$ and longer $\tau$. The modified SV equation usable for monitoring these analytes when the reactions take place in a closed cell is given by [25] 


$$
I_{0} / I=\tau_{0} / \tau=1+K_{S V} \times\left\{[\mathrm{DO}]_{\text {initial }}-[\text { analyte }]_{\text {initial }}\right\}
$$

The paper presents also aging data for a DO sensor. While commercial oxygensensitive sensing films may be suitable for monitoring oxygen for one year, long-term aging studies of the oxygen-sensitive dye PdTPP in a PMMA film indicated a decrease of the average PL quenching rate by a factor larger than four during a nine-month aging period [13]. Previously, some sol-gel sensor materials with embedded tris(4,7-diphenyl-1,10phenanthroline) ruthenium(II) $\left(\mathrm{Ru}(\mathrm{dpp})_{3}\right)$ demonstrated long-term stability over an 11-month period [11]. However, Pt- and Pd-porphyrins, with their longer $\tau_{0}$ and consequently increased detection sensitivities, are typically embedded in polymeric films, which seem to behave differently from some of the sol-gel-based films.

As the long-term stability appears to be film-related, and in previous long-term stability studies $I$ was measured for monitoring gas-phase oxygen, we studied the long-term behavior of a DO sensor, monitoring $\tau$. Monitoring $\tau$ minimizes issues related to changes in the intensity of the excitation source, background light, and minor changes in the sensor film itself. The value of $\tau$ was monitored over a period of about one year, with the PtOEP:TiO $2: P S$ sensing film continually immersed in water.

\section{Experimental}

\subsection{Materials}

OLEDs: $\sim 15 \Omega /$ square indium tin oxide (ITO)-coated glass was obtained from Colorado Concept Coating, LLC., (N,N'-diphenyl-N,N'-bis(1-naphthylphenyl)-1,1'biphenyl-4,4'-diamine ( $\alpha$-NPD), coumarin (C545T), and tris(quinolinolate) Al ( $\left.\mathrm{Alq}_{3}\right)$ were 
obtained from H. W. Sands. copper phthalocyanine $(\mathrm{CuPc})$ and $\mathrm{LiF}$ were obtained from Sigma-Aldrich.

Sensing elements: PtOEP was obtained from H. W. Sands, PS, molecular weight 45,000, from Sigma-Aldrich, and toluene from Fisher Scientific. $\mathrm{TiO}_{2}$ nanoparticles, Ti-Pure R-706, with a $360 \mathrm{~nm}$ average diameter, were obtained from DuPont, glucose was purchased from Fisher Scientific, glucose oxidase (GOx) from Aspergillus niger and L-lactate from Sigma-Aldrich. Stabilized lactate oxidase (LOx) from Aerococcus viridians was obtained from Applied Enzyme Technology Inc. (Pontypool, UK). All chemicals were used as received.

\subsection{Procedures}

OLED fabrication: OLED pixels were fabricated by thermal evaporation of organic layers on $\sim 150 \mathrm{~nm}$ thick ITO, which was treated as previously described [10]. The organic layers consisted of a $5 \mathrm{~nm}$ thick $\mathrm{CuPc}$ hole injecting layer and a $50 \mathrm{~nm}$ thick NPD hole transport layer (HTL). For the green OLEDs, with peak emission at $\sim 530 \mathrm{~nm}$, the $\sim 45 \mathrm{~nm}$ thick emitting and electron transport layer (ETL) was either $\mathrm{Alq}_{3}$ or $20 \mathrm{~nm} 1$ wt.\% C545Tdoped $\mathrm{Alq}_{3} / 25 \mathrm{~nm} \mathrm{Alq}$. An 8-10 $\AA \mathrm{LiF}$ buffer layer was deposited on the organic layers followed by the $\sim 120 \mathrm{~nm}$ thick Al cathode. The OLEDs were encapsulated with glass covers glued using Torr Seal epoxy to prevent water and $\mathrm{O}_{2}$ exposure. The total thickness of the OLEDs, excluding the substrate and cover glass was thus $<0.4 \mu \mathrm{m}$. The green emitting LEDs with peak at $525 \mathrm{~nm}$ were obtained from Cree.

Sensing elements preparation: The sensing films for gas phase/DO sensors were prepared by drop casting 50-60 $\mu \mathrm{L}$ of toluene solution, which contained $1 \mathrm{mg} / \mathrm{mL}$ PtOEP, 
$\sim 40 \mathrm{mg} / \mathrm{mL} \mathrm{PS}$, and $0-8 \mathrm{mg} / \mathrm{mL} \mathrm{TiO}_{2}$, on cleaned glass slides. Prior to drop casting, the dye solution was ultrasonicated to uniformly suspend it and the $\mathrm{TiO}_{2}$ nanoparticles. The solutions were spread on the slides to generate typically $7-8 \mu \mathrm{m}$ thick films. The resulting films were allowed to dry in the dark at ambient temperature for at least 24 hours.

For glucose and lactate sensors, $20 \mu \mathrm{L}$ of PtOEP:PS in toluene solution were drop cast into an $8 \mathrm{~mm}$ in diameter cylindrical reaction cell, generating $\sim 7 \mu \mathrm{m}$ thick films at the bottom of the $200 \mu \mathrm{L}$ reaction cell.

Monitoring the PL decay time: The PL decay curves, at different levels of gas phase $\mathrm{O}_{2}$ or DO, were obtained following a typical $100 \mu$ s OLED or LED excitation pulse. $\tau$ was extracted from the decay curves using a single exponential and a stretched exponential fit. When using the latter, $\tau_{k w w}$ and $\beta$ were obtained by the least-squares fit of Eq. (3) to the measured curve, and $\left\langle\tau>, \sigma^{1 / 2}\right.$, and $w$ were then calculated from Eqs. (4) and (5).

Instrumentation: OLED arrays were fabricated by thermal vacuum evaporation of the organic layers in a deposition chamber (background pressure $\sim 1-2 \times 10^{-6}$ Torr) installed in an Ar-filled glove box (typical $\mathrm{O}_{2}$ levels $\sim 1 \mathrm{ppm}$ ). The OLEDs were driven by an AVTECH AV-1011B pulse-generator.

The PL was monitored with a Hamamatsu R6060 photomultiptier tube (PMT) operated at $\sim 950 \mathrm{~V}$ or a Si photodiode. The photodetector (PD) was typically used in the "back-detection" geometry, collecting the PL passing through the gaps between the OLED pixels that were used for excitation. Front detection geometry, with the sensor film sandwiched between the excitation source and the PD, was used in the LED-based long-term measurements. We note that the OLED and LED excitation sources, using either the back 
detection or the front detection geometries, resulted in comparable results, with the OLED/back detection design being more compact and flexible.

The glucose and lactate measurements were performed in a sealed reaction well to prevent replenishing of oxygen from the ambient following the oxidation reactions. Calibration curves were based on the modified Stern-Volmer relation (Eq. 7) [25].

Gas-phase $\mathrm{O}_{2}$ and DO were monitored using flowing oxygen/argon mixtures, as previously described [16]; replacing Ar with $\mathrm{N}_{2}$ had no effect on the results. Mixing was achieved by means of mass flow controllers, where the flow rates of the oxygen and argon varied, while maintaining a constant total flow rate, thus generating varying oxygen partial pressures.

Measurements at temperatures above ambient were performed using a Fisher Scientific Isotemp incubator. The incubator housed the sensing element and flow cell, and the gas carrying tubing, which was extended to assure its temperature equilibration. Measurements at $0^{\circ} \mathrm{C}$ were performed with the sample housing immersed in a mixture of ice and water.

\section{Results and discussion}

As mentioned, in this study the sensing film was PtOEP:PS or PtOEP:TiO 2 :PS. Optimized results were obtained for $\sim 7-8 \mu \mathrm{m}$ thick films of a PtOEP:PS ratio of 1:40 in the toluene solution used for film preparation. We have further shown that doping the PtOEP:PS films with $\mathrm{TiO}_{2}$ particles (360 nm in diameter) significantly increases the PL intensity, probably a result of an increased optical path of the excitation light within the film due to its scattering by the particles that have a high dielectric constant [17]. This increased optical 
path results in increased absorption by the PtOEP. Thus, we typically use films of PtOEP:TiO 2 :PS ratios of 1:1:40 to 1:3:40 in the toluene solutions used for film preparation.

All studies were performed by monitoring the PL decay time following an excitation pulse. The excitation sources were green LED or (typically two) OLED pixels. OLED-based sensing is a growing research field [26], due to attractive attributes of the OLEDs. These attributes include ease of fabrication on glass or plastic substrates that makes them compatible with microfluidic architectures, uniquely simple integration with the sensing component, small and flexible size, and adaptable design that includes single- or combinatorially fabricated [27] multiple-color pixel arrays. The latter can be used for detection of multiple analytes on a compact structure. OLED-based sensors are expected to be disposable and are therefore not as susceptible to the long-term stability issue of the organic devices, which remains a challenge.

We note that in this work the drive to use the stretched exponential analysis was the non-exponential PL decay curves following exposure to $\mathrm{O}_{2}$, though single exponent analysis often resulted in linear SV plots. Importantly, in all of the experiments conducted in a pure Ar or $\mathrm{N}_{2}$ atmosphere, the PL decay curve was in excellent agreement with a simple exponential decay curve (correlation coefficient $R^{2}$ well over 0.99 ; see below), as was also the case in other studies $[17,29]$.

As mentioned, a constant value of $\beta$, typically 0.5 [9], was previously used to analyse the PL decay in $\mathrm{O}_{2}$ sensors. In the present study, however, a constant value of $\beta$, e.g., 0.5 , 0.75 , or 0.85 , was not suitable to describe the PL decay curves over the whole analysis range of $0-100 \% \mathrm{O}_{2}$, as it resulted in poor fits either at $0 \%$ and (at least) $100 \% \mathrm{O}_{2}$. As a result, and 
since the PL decay curve was a simple exponent in the absence of $\mathrm{O}_{2}$, independent of the environment whether $\mathrm{N}_{2}$ or Ar, Eq. (3) was used with $\tau_{k w w}$ and $\beta$ as the fitting parameters. As shown, the resulting $\beta$ was found to be strongly dependent on $\left[\mathrm{O}_{2}\right]$.

\subsection{Gas-phase sensors}

Fig. 1 shows two linear SV plots for a PtOEP:PS film using a green pulsed OLED excitation source. One plot was obtained using single-exponential analysis, while the other was obtained using the stretched exponential analysis. As seen, the latter resulted in a larger detection sensitivity

$$
S \equiv \tau_{0} / \tau\left(100 \% \mathrm{O}_{2}\right)
$$

Importantly, for $100 \% \mathrm{O}_{2}, R^{2}$ values for the single and stretched exponential analyses of the PL decay curves were 0.981 and 0.992 , respectively. As shown below for a DO sensor, such differences in $R^{2}$ are significant. At $0 \% \mathrm{O}_{2}, R^{2}$ values for both types of analysis were 0.997 , as $\beta$ was nearly 1 .

Based on the reproducible, excellent single exponential fits at $0 \% \mathrm{O}_{2}$, it is believed that in the PtOEP:PS and PtOEP:TiO 2 :PS films the interaction of the dye molecules with microheterogeneous PS sites is not the main reason for the non-exponential behavior. As the non-exponential behavior is evident only in the presence of the $\mathrm{O}_{2}$ quencher and is dosedependent (see below), it probably implies that a microheterogeneity-induced distribution of the rates of the $\mathrm{O}_{2}$ :dye collisions is responsible for this behavior. In principle, non-uniform accessibility of the dye molecules to the quencher and different oxygen diffusion rates in the matrix due to its heterogeneous microstructure will likely result in deviations from an exponential PL decay time by affecting the frequency of the quenching collisions. 
We note that $S$ values ranging from $\sim 14$ to $\sim 40$ have been obtained for PtOEP-based gas-phase $\mathrm{O}_{2}$ sensors $[16,17]$. These variations are mostly due to the $\tau$ value obtained for $100 \% \mathrm{O}_{2}$, which unexplainably varies from $\sim 3$ to $\sim 8 \mu$ s in seemingly comparable films.

Fig. 2 shows the dependence of the stretching factor $\beta$ (for the film of Fig. 1) on the oxygen level. As seen, $\beta$ changes from 1 at $0 \% \mathrm{O}_{2}$ to $\sim 0.5$ at $100 \% \mathrm{O}_{2}$. The figure shows also the $\mathrm{O}_{2}$-dependent $\beta$ for a film that was additionally doped with $\mathrm{TiO}_{2}$ particles, $360 \mathrm{~nm}$ in diameter. The data shown is for a fresh PtOEP:TiO 2 :PS film of 1:2:40 component ratio. As seen, the values of $\beta$ changed from 1 to $\sim 0.65$. This behavior was the same whether the component wt ratio was $1: 2: 40$ or $1: 8: 40$. The dose-dependent $\beta$ can be explained by the potential existence of multiple $\mathrm{O}_{2}$ trapping sites, albeit possibly shallow, in PS. These different traps, whose effect depends on $\left[\mathrm{O}_{2}\right]$, result in different frequencies of dye: $\mathrm{O}_{2}$ collisions. As $\left[\mathrm{O}_{2}\right]$ increases, more of these sites are accessed by the quencher, resulting in a dose-dependent $\beta$, with a relative distribution width that increases with increasing $\left[\mathrm{O}_{2}\right]$. This explanation is equivalent to assuming a dose-dependent, varying $\mathrm{O}_{2}$ diffusion rate within the PS matrix.

Fig. 3 shows $\sigma^{1 / 2}$ and $w$ for PtOEP:PS and PtOEP:TiO ${ }_{2}$ :PS films vs $\left[\mathrm{O}_{2}\right]$. The observed smaller values of $\sigma^{1 / 2}$ and the narrower relative distributions of the PL decay times for the film doped additionally with titania particles indicate a change in the microstructure. Clearly, the $\mathrm{TiO}_{2}$ particles that result in longer PL decay times, reduce the rate of dye: $\mathrm{O}_{2}$ collisions. This may be a result of reduced accessibility of the dye molecules to the quencher, or slower diffusion of the quenching $\mathrm{O}_{2}$, which may become trapped in e.g., voids generated in the particle-doped matrix or on the $\mathrm{TiO}_{2}$ surfaces [29, 30-32]. 
Increasing the film thickness by using 50,100 , and $150 \mu \mathrm{L}$ of the component mixture for film fabrication on a constant substrate area did not affect the values of $\beta$. This behavior may indicate comparable microstructures in all films and consequently comparable $\mathrm{O}_{2}$ diffusion and dye: $\mathrm{O}_{2}$ collision frequencies. The values of $S$ for the films prepared from 50, 100 , and $150 \mu \mathrm{L}$ solution were $14.5,12.8$ and 13.6 , respectively, when using the single exponential analysis, and 18.5, 13.6, and 14.9, respectively, when using the stretched exponential analysis. The pulsed excitation was performed with a green $\mathrm{Alq}_{3}$-based OLED biased at $22 \mathrm{~V}$.

\subsection{Dissolved oxygen sensors}

When monitoring DO with the 1:3:40 PtOEP:TiO ${ }_{2}: \mathrm{PS}$ film, the SV equation is typically obeyed, resulting in linear calibration. The values of $R^{2}$ obtained when fitting the PL decay curves with a single exponent exceed 0.99 for low oxygen concentrations, but deteriorate as the oxygen level increases. Fig. 4 shows a decay curve obtained for $40 \mathrm{ppm}$ DO at $23^{\circ} \mathrm{C}$ (the [DO] in equilibrium with an almost pure $\mathrm{O}_{2}$ atmosphere) with both the exponential and stretched exponential fits plotted over the experimental data. The residuals, which show the difference between the experimental and calculated points, for each case are also shown. The excitation source used was a green LED. As seen, the fit is considerably better when using the stretched exponential analysis with $R^{2}$ improving from 0.986 to 0.995 . For comparison, Fig. 4 shows also the single exponential fit and residuals for a PtOEP:PS film in the absence of the quencher. The film was excited by a coumarin-doped Alq ${ }_{3}$-based OLED. Similarly excellent fits were obtained when using PtOEP:TiO 2 :PS films, independent of the excitation source. 
Fig. 5a shows the change in $\tau$ for a 1:3:40 $\mathrm{PtOEP}_{\mathrm{TiO}_{2}}: \mathrm{PS}$ film monitored over a period of about one year. The film was immersed in water, in the dark, for the whole measurement period. The PL decay curve was monitored using a green LED as the excitation source and $\tau$ was obtained using a single exponential analysis. The temperature was $\sim 23^{\circ} \mathrm{C}$ and the DO level $\sim 8.6 \mathrm{ppm}$. As seen, in the first $\sim 25$ days $\tau$ reduced from $\sim 28$ to $\sim 24 \mu \mathrm{s}$. It stabilized toward the end of the measurement period (during the last $\sim 100$ days, $\tau$ stabilized at $23.3 \pm 0.85 \mu \mathrm{s})$, but by then the film appeared lighter in color, possibly due to photo bleaching [31], though the film was exposed to light only briefly for each measurement, and/or some dye leaching. Fig. 5b shows $\langle\tau\rangle=21.0 \pm 1.5 \mu \mathrm{s}$, obtained using the stretched exponential analysis, which improved $R^{2}$, as was the case for the gas-phase sensor. The average value of $\beta$ over the whole period was $0.67 \pm 0.05$, however, the scatter in its value was stronger during the first $\sim 150$ days. Fig. 6 shows $\beta$ during the last $\sim 160$ days of the measurement.

The effect of the temperature on $\beta$ in the range 0 to $60{ }^{\circ} \mathrm{C}$ was small; no clear trend was observed. Fig. 7 shows the values of $\beta$ for a $\sim 6$-months old film for $0-100 \%$ gas-phase $\mathrm{O}_{2}$ at equilibrium with water (i.e., 0 to $40 \mathrm{ppm}$ DO). As seen, $\beta$ varied from 1 to $\sim 0.5$. Although Fig. 7 shows that for any value of $\left[\mathrm{O}_{2}\right] \beta$ at $60{ }^{\circ} \mathrm{C}$ is lower than at $20{ }^{\circ} \mathrm{C}$, the values at $0{ }^{\circ} \mathrm{C}$ (not shown) were similar to those at $60^{\circ} \mathrm{C}$, and those at $40{ }^{\circ} \mathrm{C}$ were similar to those at $20{ }^{\circ} \mathrm{C}$. We note that the values of $\tau$ were temperature-dependent, decreasing with increasing temperature [14]. However, the decrease at temperatures up to $55^{\circ} \mathrm{C}$ was small. The effect of temperature on the detection sensitivity, $\tau, \beta$, and the decay time distribution needs further evaluation. 
Fig. 8 shows the SV lines obtained after 6 and $\sim 12$ months since film preparation with the film, as mentioned, constantly immersed in water. As seen, the sensitivity reduced over time by a factor of 1.7 , from 14.8 after 6 months to 8.6 after one year. The change in the PL decay rate over one year is smaller than that reported earlier for a PdTPP in a PMMA matrix [13], and is probably dependent on the matrix, dye, and the film preparation procedure. The change in the film's detection sensitivity and calibration over time indicate the need for sensor calibration or preferably periodical film replacement in commercial PL-based DO sensors. In the gas-phase, films were stable for at least four months without change in performance.

\subsection{Glucose and lactate sensors}

PL-based glucose, lactate, and ethanol sensors are all based on monitoring the DO concentrations following their oxidation reactions in the presence of oxygen and their specific oxidase enzymes. In this work, the results obtained for reactions performed in closed cells were analyzed. In that case, Eq. (7) is obeyed with the final DO level being equal to the difference between the initial DO and analyte concentrations. The differences in the values of $\tau$ obtained using both types of analysis for glucose and lactate sensors were small, as expected for the low DO levels ranging from 0 to $\sim 8.6 \mathrm{ppm}$.

The calibration lines for glucose, based on Eq. (7), are shown in Fig. 9. The value of $\beta$ changed from 1 at $\sim 0.25 \mathrm{mM}$ glucose (practically, a solution depleted of DO following its consumption in the oxidation reaction in a closed cell, where there is no replenishing of oxygen from the ambient) to $\sim 0.7$ at $\sim 0.02 \mathrm{mM}$. 
Fig. 10 shows the relative distribution width $w$ (see Eq. (5)) of the PtOEP phosphorescence decay times at various levels of DO for a lactate sensor. As expected, $w$ decreases with decreasing DO level (increasing lactate concentration) as $\langle\tau\rangle$ increases. Comparable results were obtained for glucose when using the same film, however, in general, the decay-time distributions were film-dependent and thus, similar only qualitatively for different films. In particular, these distributions were dependent on the films' age.

\section{Concluding remarks}

The results presented clearly indicate that the stretched exponential analysis is well suited to analyze the PL decay kinetics for sensors for gas-phase oxygen, DO, and glucose and lactate. The analysis provides insight into the nature of the dye-doped films and their interactions with DO. The use of a single value of $\beta$ for a given film, at various $\left[\mathrm{O}_{2}\right]$, however, resulted in poor fits of the PL decay curves. The deviation of the PL decay curves from exponential behavior increased with increasing oxygen level; single-exponential analysis, in contrast, is similarly suitable in the absence of the quencher. The single exponential decay in the absence of the quencher together with the effects of titania doping indicate that a distribution of quencher:dye collision rates is responsible for the stretched exponential behavior. This distribution results from the films' microheterogeneity that affects the $\mathrm{O}_{2}$ diffusion and accessibility of the dye molecules to it. It is speculated that a range of $\mathrm{O}_{2}$ shallow trapping sites, with dose-dependent occupancy, can lead to the observed behavior and that the variation of $\beta$ with $\left[\mathrm{O}_{2}\right]$ may be the result of multiple trapping of the diffusing $\mathrm{O}_{2}$ quenchers. Overall, by treating the stretching factor $\beta$ for PtOEP:PS as a variable parameter, 
it is found to vary from 0.5 to 1 when the oxygen level changes from 100 to $0 \%$; for PtOEP:TiO 2 :PS in the gas-phase it was in the range 0.65 to 1.

Long-term stability studies of the DO sensor indicated visible changes in the sensing film, though the film was still usable following one year of immersion in water with frequent measurements of the PL decay time. Significant scattering of $\tau$ was observed, which is partially attributed to measurements at different points on the film itself. The detection sensitivity was practically unchanged during the first six months, but was reduced by a factor of 1.7 at the end of the one-year measurement period. As such, PL-based DO sensors should be re-calibrated if used beyond six months; preferably, the sensor film should be replaced, in particular when monitoring $\mathrm{O}_{2}$-induced changes in the PL intensity rather than lifetime.

\section{Acknowledgements}

Ames Laboratory is operated by Iowa State University (ISU) for the United States Department of Energy (USDOE) under Contract DE-AC 02-07CH11358. This work was partially supported by the Director for Energy Research, Office of Basic Energy Sciences, USDOE, and by NSF Grant IIP 0724090.

\section{References}

[1] E.R.Carraway, J.N. Demas, B.A. DeGraff, and J.R. Bacon, Anal. Chem., 63 (1991) 337342.

[2] W. Xu, R.C. McDonough, B. Langsdorf, J.N. Demas, and B.A. DeGraff, Anal. Chem., 66 (1994) 4133-4141.

[3] J.N. Demas, B.A. DeGraff, and W. Xu, Anal. Chem., 67 (1995) 1377-1380.

[4] S. Draxler, M.E. Lippitsch, I. Klimant, H. Kraus, and O.S. Wolfbeis, J. Phys. Chem., 99 (1995) 3162-3167.

[5] Z. Rosenzweig, R. Kopelman, Anal. Chem., 67 (1995) 2650-2654. 
[6] W. Trettnak, W. Gruber, F. Reininger, I. Klimant, Sensors and Actuators B, 29 (1995) 219-225.

[7] S. Draxler and M.E. Lippitsch, Anal. Chem., 68 (1996) 753-757.

[8] C. McDonagh, B. D. MacCraith, A. K. McEvoy, Anal. Chem., 70 (1998) 45-50.

[9] M.L. Bossi, M.E. Daraio, P.F. Aramendia, J. Photochem. Photobiol., 120 (1999) 15-21.

[10] V. Savvate'ev' Z. Chen-Esterlit, J. W. Aylott, B. Choudhury, C.-H. Kim, L. Zou, J. H. Friedl, R. Shinar, J. Shinar, R. Kopelman, Appl. Phys. Lett., 81 (2002) 4652-4654.

[11] Y. Tang, E.C. Tehan, Z. Tao, and F.V. Bright, Anal. Chem., 75 (2003) 2407-2413 and references therein.

[12] R.N. Gillanders, M.C. Tedford, P.J. Crilly, R.T. Bailey, Anal. Chim. Acta, 502 (2004) 16.

[13] K. Oige, T. Avarmaa, A. Suisalu, and R. Jaaniso, Sens. Actuators B, 106 (2005) 424430.

[14] P. Hartmann, Anal. Chem., 72 (2000) 2828-2834.

[15] K.A. Kneas, J.N. Demas, B. Nguyen, A. Lockhart, W. Xu, B.A. DeGraff, Anal. Chem., 74 (2002) 1111-1118.

[16] R. Shinar, Z. Zhou, B. Choudhury, J. Shinar, Anal. Chim. Acta, 568 (2006) 190-199.

[17] Z. Zhou, R. Shinar, A.J. Allison, J. Shinar, Adv. Funct. Mater., 17 (2007) 3530-3537.

[18] G. Williams and D. C. Watts, Trans. Faraday Soc., 66 (1970) 80 - 85.

[19] J. T. Bendler and M. F. Shlesinger, Macromol., 18 (1985) 591 - 592.

[20] C.P. Lindsey, G.D. Patterson, J. Chem. Phys., 73 (7) (1980) 3348-3357.

[21] K.C.B. Lee, J. Siegel, S.E.D. Webb, S. Leveque-Fort, M.J. Cole, R. Jones, K. Dowling, M.J. Lever, and P.M.W. French, Biophys. J., 81 (2001) 1265-1274.

[22] Z. Rosenzweig and R. Kopelman, Sens. Actuat. B, 35-36 (1996) 475-483.

[23] S. de Marcos, J. Galindo, J. F. Siera, J. Galban, and J. R. Castillo, Sens. and Actuat. B 57 (1999) 227-232.

[24] B. Choudhury, R. Shinar, J. Shinar, J. Appl. Phys., 96 (2004) 2949-2954. 
[25] Y. Cai, R. Shinar, Z. Zhou, C. Qian, and J. Shinar, Sens. and Actuators B 134 (2008) 727-735.

[26] J. Shinar and R. Shinar. J. Phys. D., 41(13) (2008) 133001-133027 and references therein.

[27] L. Zou, V. Savvate'ev, J. Booher, C.-H. Kim, and J. Shinar, Appl. Phys. Lett., 79 (2001) $2282-2284$.

[28] K.O. Cheon and J. Shinar, Appl. Phys. Lett., 83 (10) (2003) 2073-2075.

[29] X. Lu, I. Manners, and M. A. Winnik, Macromolecules, 34 (6) (2001) 1917-1927.

[30] X. Lu, M. A.Winnik, Chem. Mater., 13 (2001) 3449-3463.

[31] J. L. Pfeifer, T. A. Libsch, and H. P. Wertheimer, Soc. Automot. Eng., 840 (1985) 18481855.

[32] A. Takami, Ceram. Bull., 67 (1988) 1956-1960. 


\section{Figures}

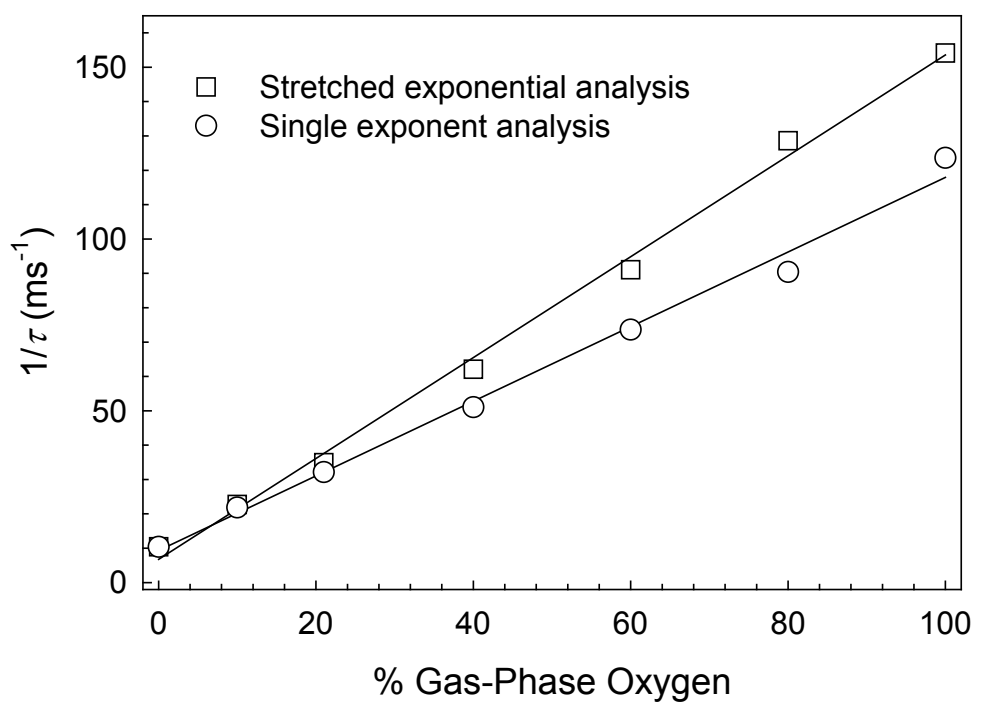

Fig. 1. Gas-phase SV plots using single exponent and stretch exponential analyses of the PL decay curves for a PtOEP:PS film excited by a pulsed green OLED. $1 /<\tau\rangle$ was plotted for the stretched exponential analysis.

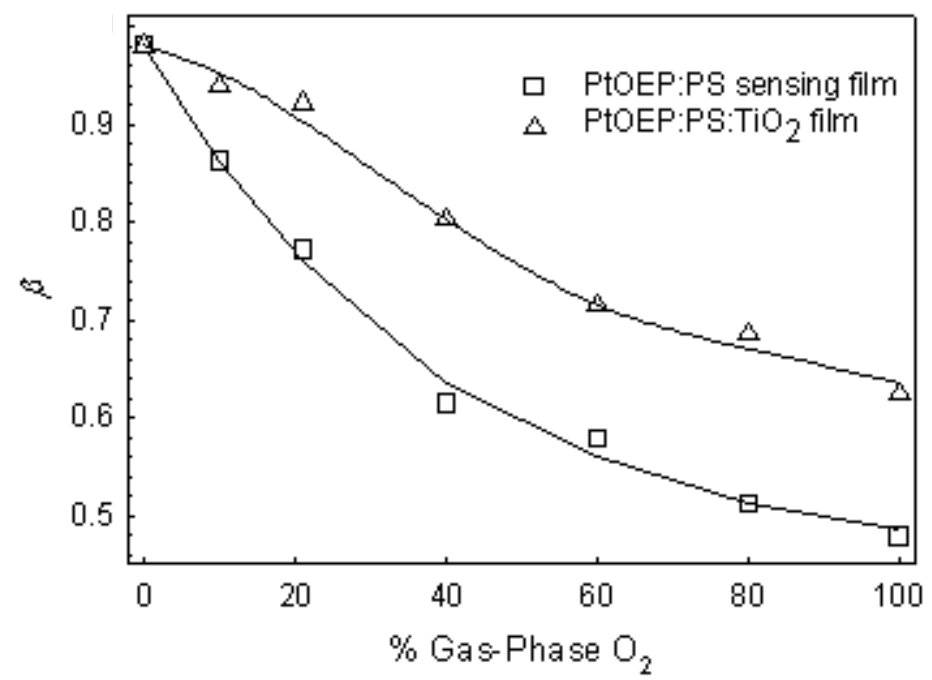

Fig. 2. The stretching factor as a function of the oxygen level for fresh PtOEP:TiO 2 :PS films of component wt ratios 1:0:40 (same film as of Fig. 1) and 1:3:40. The lines are a guide to the eye. 

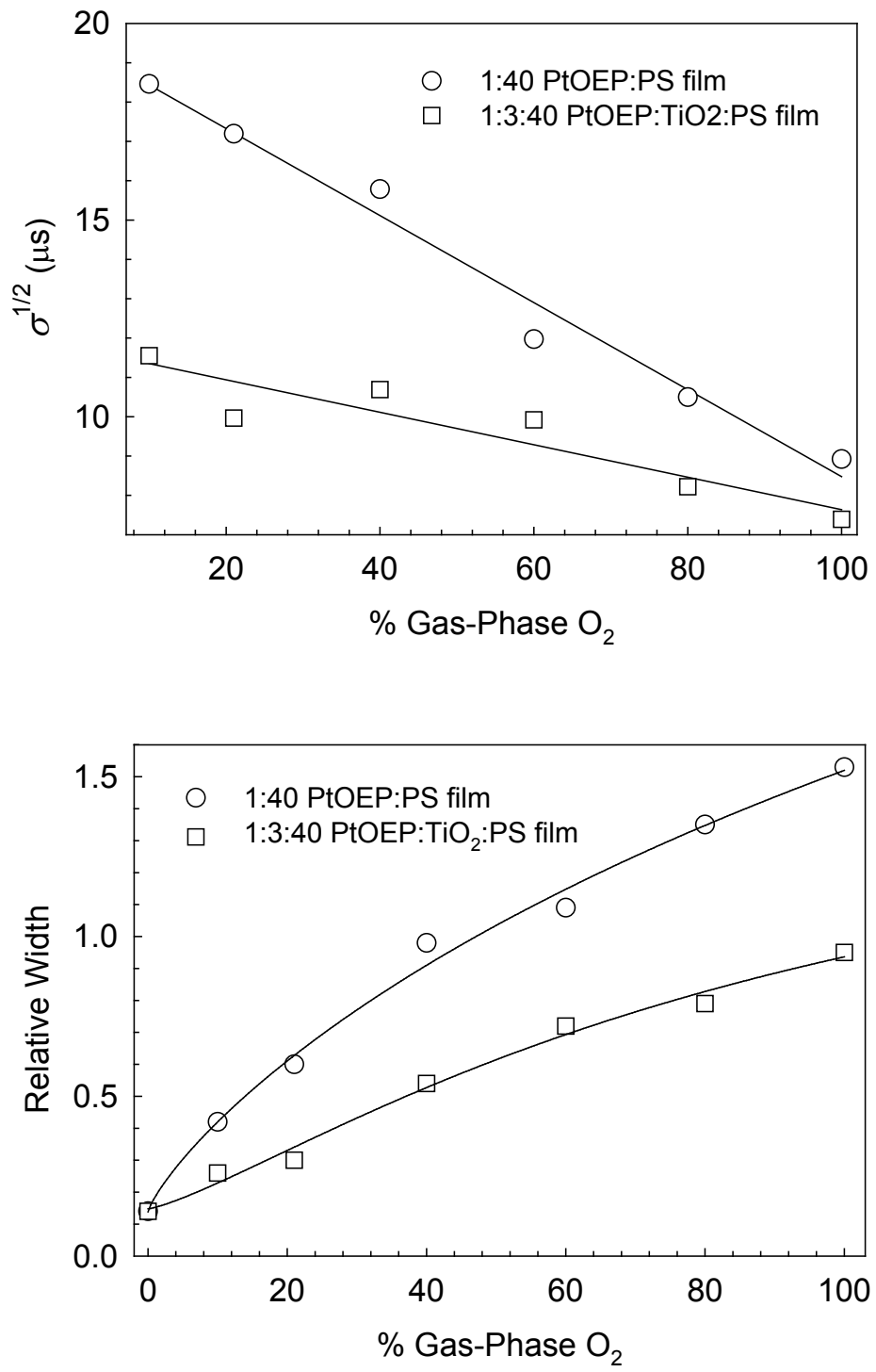

Fig. 3. The (a) square root of the variance $\sigma^{1 / 2}$ and (b) relative width $w$ (which quantify the absolute distribution width and width relative to $\langle\tau\rangle$, respectively; see Eqs. (3) $-(5))$ of the $\mathrm{PL}$ decay times for sensor films PtOEP:PS (circles) and PtOEP:TiO $2: \mathrm{PS}$ (squares), prepared from a solution containing $1 \mathrm{mg} / \mathrm{mL}$ PtOEP, $40 \mathrm{mg} / \mathrm{mL}$ PS, and 0 or $3 \mathrm{mg} / \mathrm{mL} \mathrm{TiO}$ particles, respectively. The data are for gas-phase $\mathrm{O}_{2}$ measurements. The lines are a guide to the eye. 

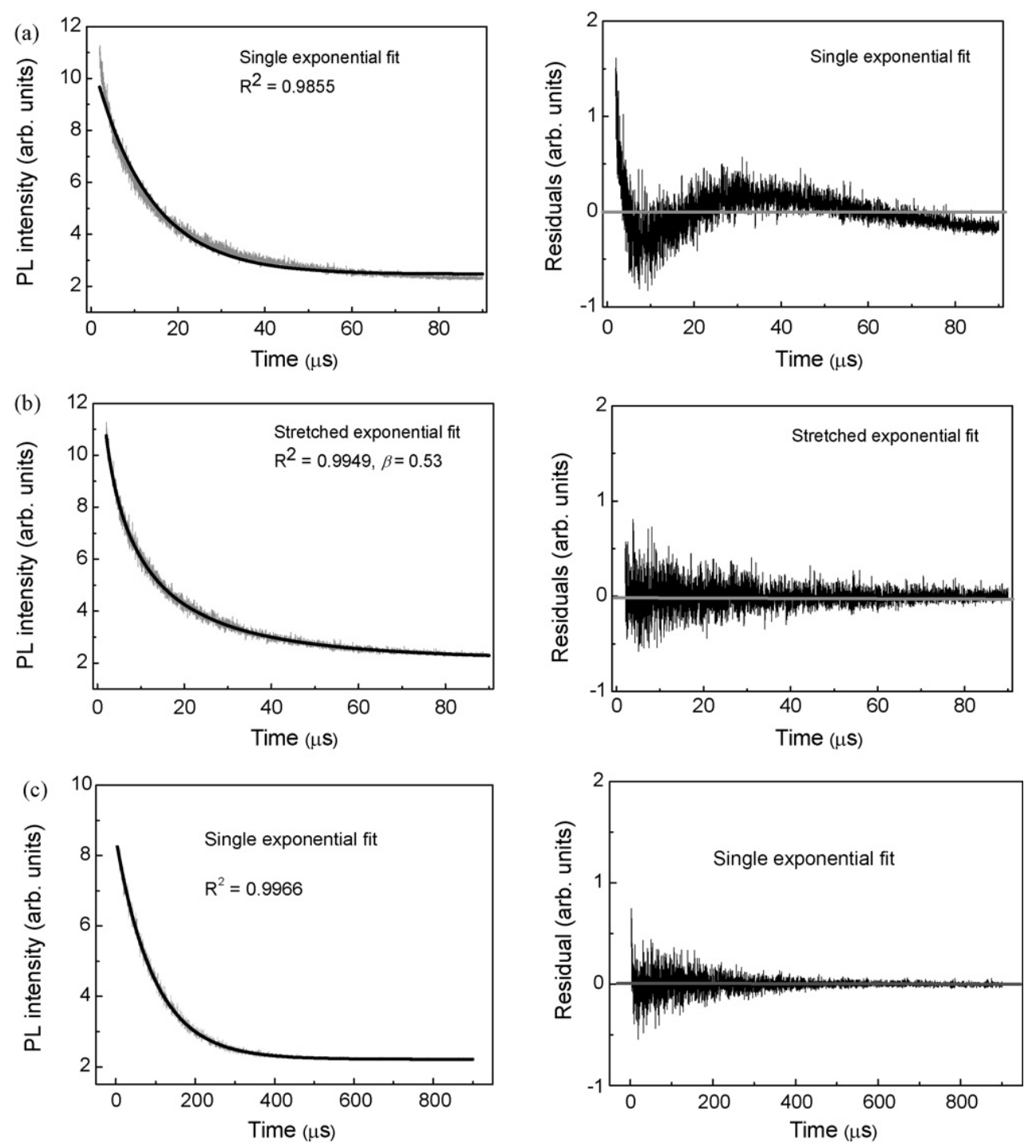

Fig. 4. Experimental decay curves following a $100 \mu$ s LED pulse and (a) the single and (b) stretched exponential analysis, for $[\mathrm{DO}]=40 \mathrm{ppm}$. The sensor film was a PtOEP:TiO $2: \mathrm{PS}$ at a component ratio of 1:3:40. (c) The experimental decay curve in pure Ar or $\mathrm{N}_{2}$, and the simple exponential fit to that curve. The sensor film was a 1:40 PtOEP: PS. The residuals (see text) for each analysis are also shown. 

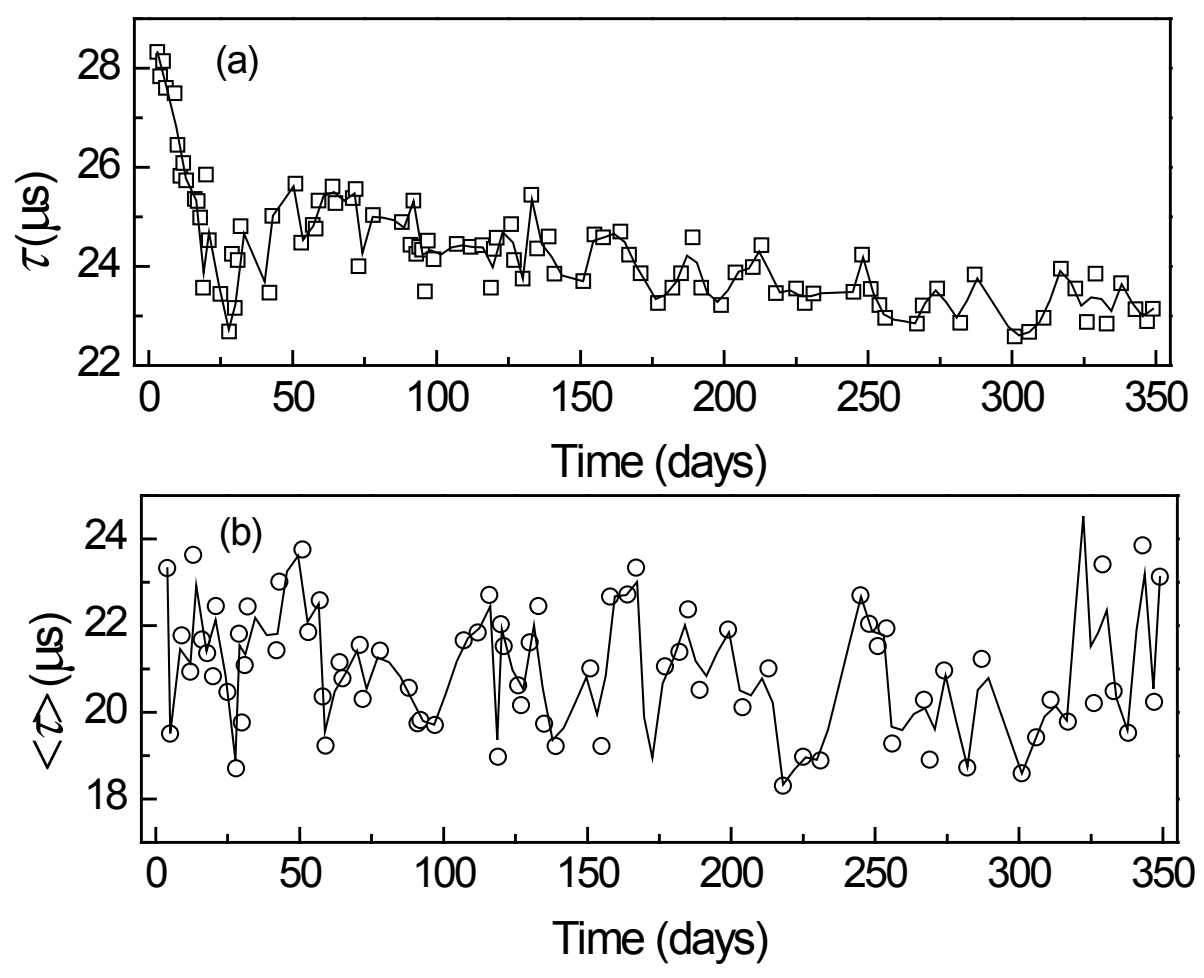

Fig. 5. The PL decay time of a 1:3:40: PtOEP:TiO $2:$ PS measured over a period of $\sim 1$ year at $\sim 23^{\circ} \mathrm{C}$ following a $100 \mu$ s pulse of a green LED (a) using a single exponential analysis of the PL decay curve (b) the average $\tau$ using stretched exponential analysis. The lines present 5 point average values. 


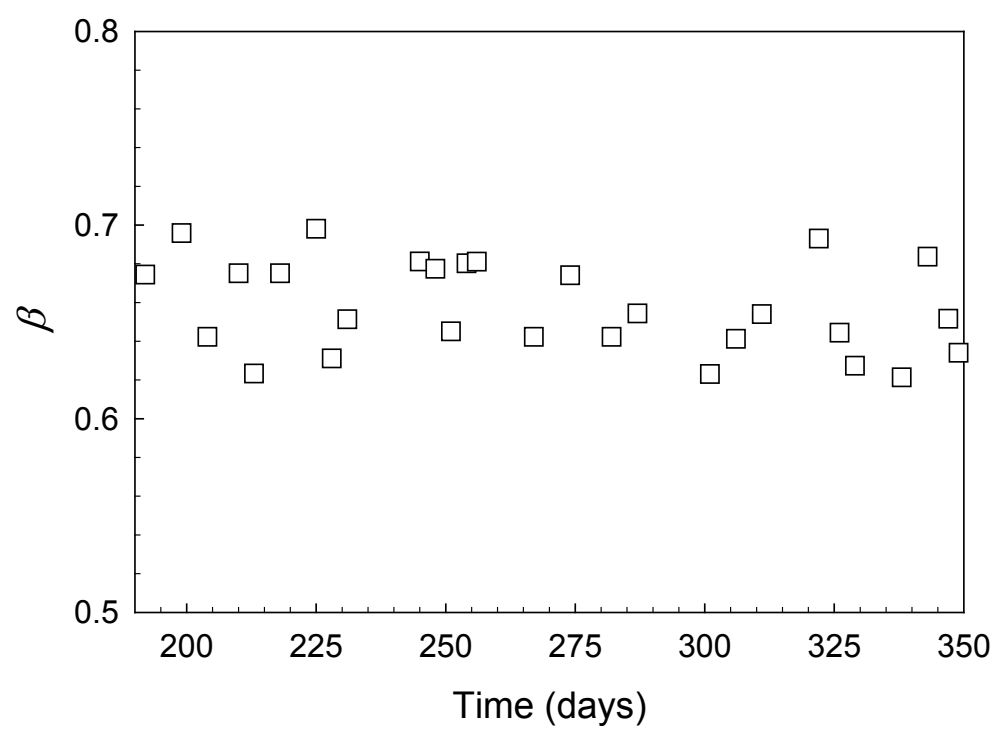

Fig. 6. The value of $\beta$ over the last $\sim 160$ days of the measurement.

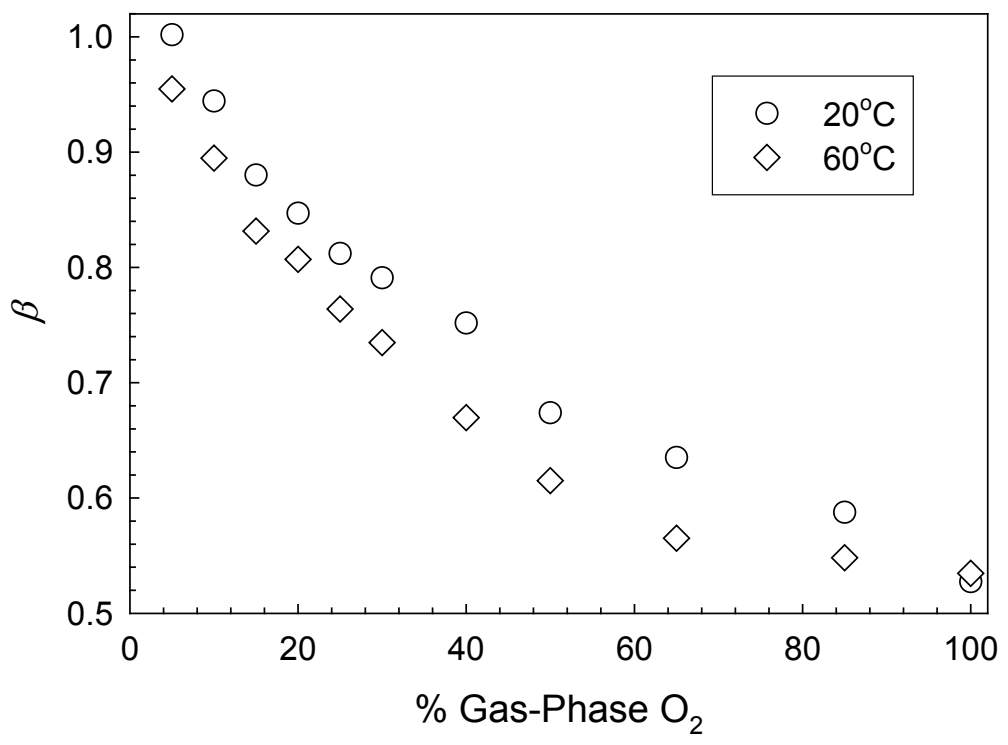

Fig. 7. Values of $\beta$ vs gas-phase $\mathrm{O}_{2}$ in equilibrium with water at 20 and $60{ }^{\circ} \mathrm{C}$ for a 6 -months old sensor continually immersed in water. The film was 1:3:40 PtOEP:TiO $2: \mathrm{PS}$. 


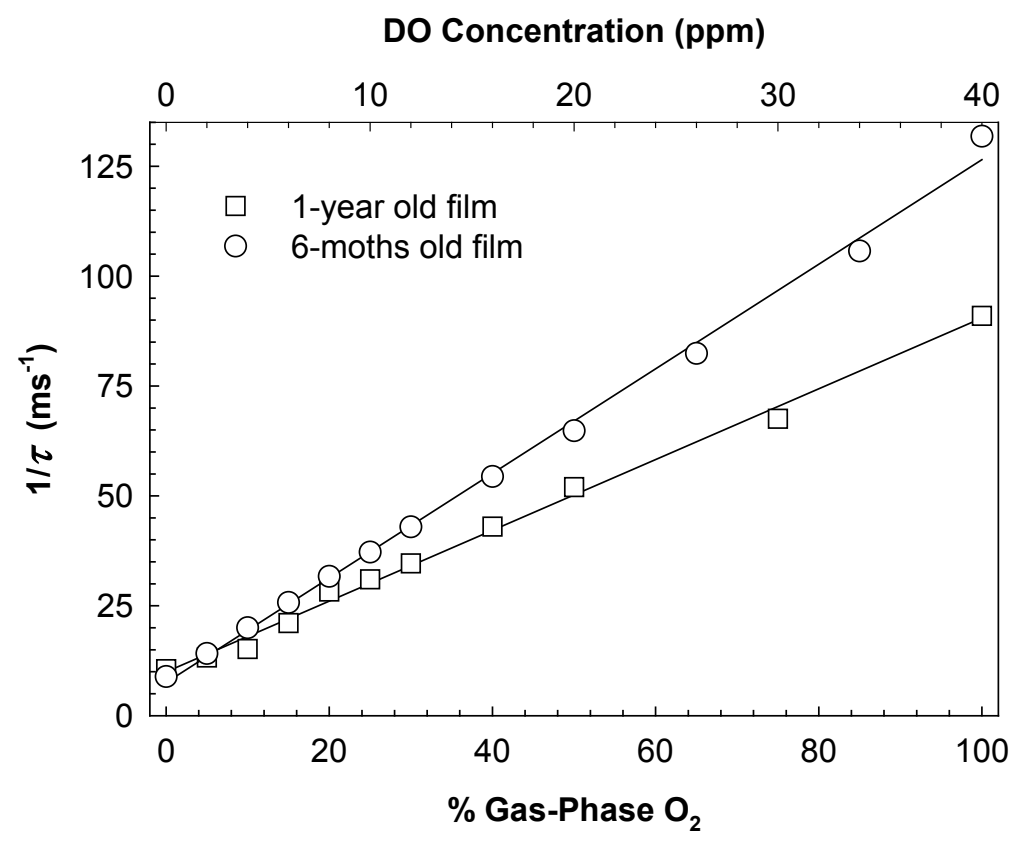

Fig. 8. Calibration lines of the DO sensor (utilizing the same 1:3:40 PtOEP:TiO $2: \mathrm{PS}$ sensor film constantly immersed in water) at different periods: circles - $\sim 6$ months old film; squares - 12 months old film.

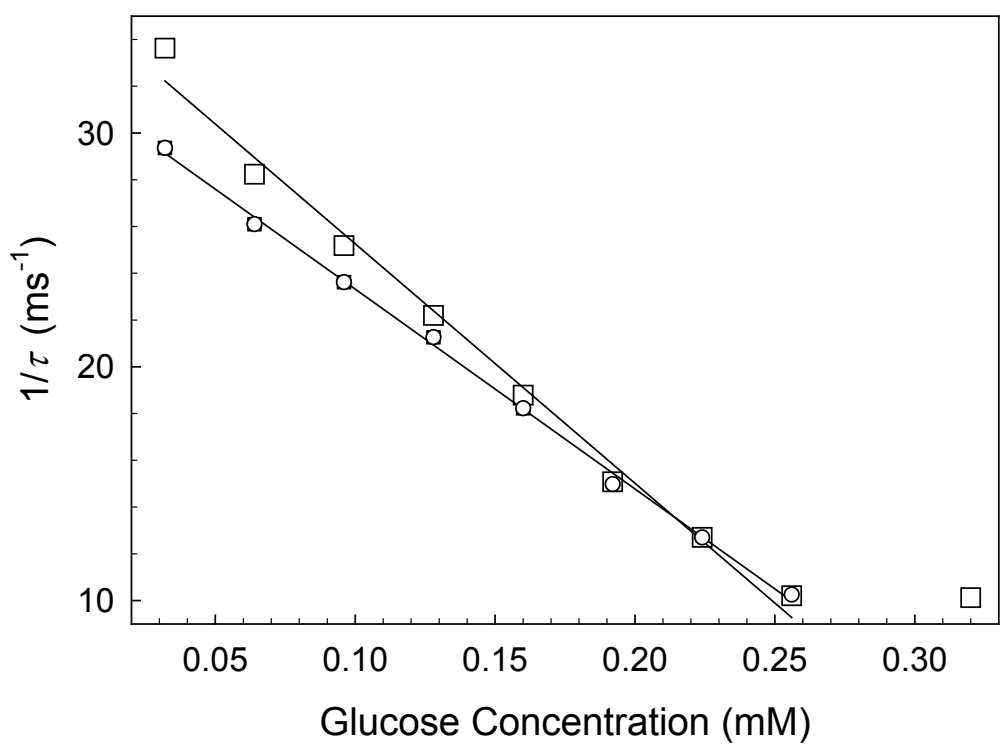

Fig. 9. The modified SV plots for a glucose sensor using (circles) single- and (squares) stretched-exponential analysis. 


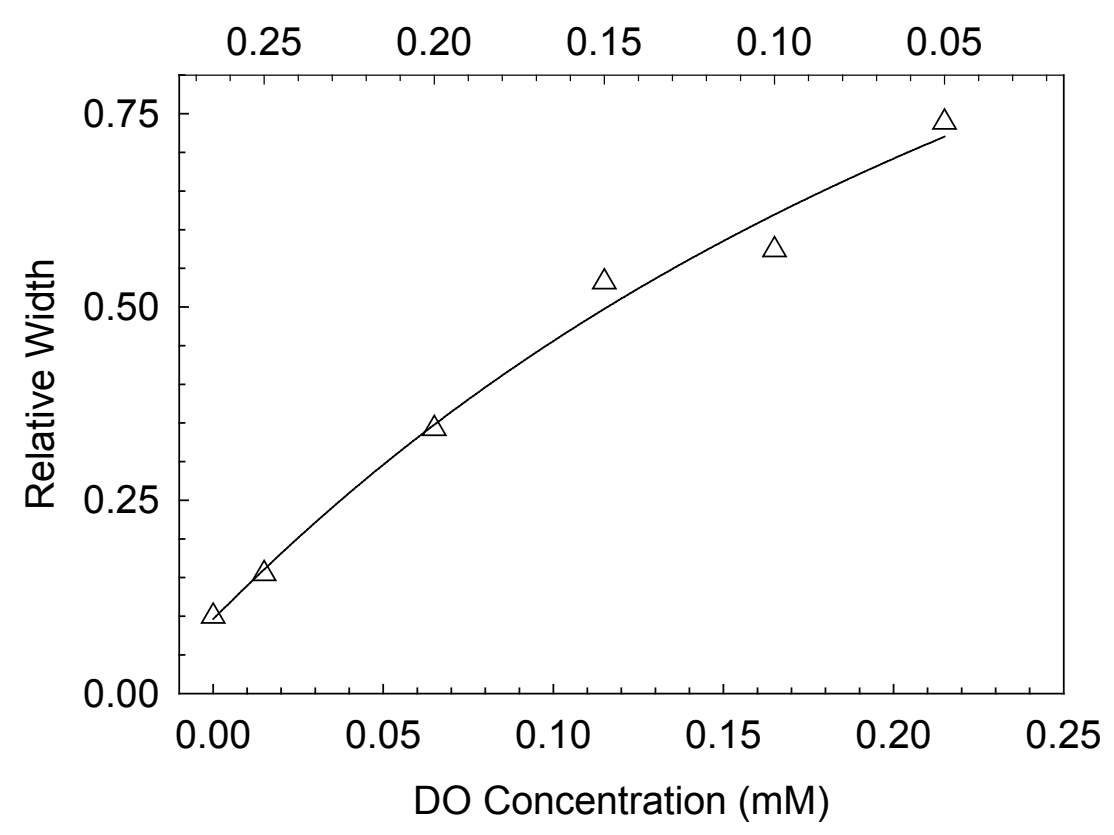

Fig. 10. The relative width $w$ of the distributions of the decay rates (see Eq. (5)) for a lactate sensor. The sensor film was 1:40 PtOEP:PS. The line is a guide to the eye. 


\title{
Chapter 7. Polythiophene-fullerene based photodetectors: tuning of spectral response and application in photoluminescence based
}

\section{(bio)chemical sensors}

\author{
A paper accepted for publication in Advanced Materials \\ K. Nalwa, Y. Cai, A. Thoeming, J. Shinar, R. Shinar, S. Chaudhary
}

\begin{abstract}
Organic electronics is attracting extensive interest in the development of low-cost and flexible devices, such as solar cells [1], light-emitting diodes (LEDs) [2], and photodetectors [3]. Recently, the use of organic electronics has been broadened toward novel devices, including photoluminescence (PL)-based (bio)chemical sensors using organic LEDs (OLEDs) as excitation sources [4]. The viability of a (bio)chemical sensing platform increases when the fabrication of all its components is simple, and they are compact and easily integratable. In this direction, an integrated platform based on OLED pixels excitation, a luminescing sensing medium, and PL-detecting organic photodetectors (OPDs) is a promising approach. This communication describes steps toward the development of such a compact sensing platform. In particular, a bulk-heterojunction OPD based on poly(3-hexylthiophene) and fullerene derivatives was engineered to be sensitive to the sensing film's PL, with a fast response time for monitoring analytes in both the PL intensity and PL decay time detection modes.
\end{abstract}

\section{Introduction}

The need for (bio)chemical sensing systems is burgeoning for various analytical problems in fields such as medicine, the environment, defense and food. Optical sensing 
techniques - most notably luminescence based - are sometimes the only ones that provide adequate sensitivity [5]. In general, luminescence-based (bio)chemical sensors require three components (excluding the electronics and readout): a fluorescing or phosphorescing sensing element, a light source that excites the PL of that sensing element, and a photodetector. Traditional light sources are lasers or LEDs that cannot be easily integrated with the other components due to size, geometrical, or operational constraints [6]. Traditional photodetection elements include charge coupled device cameras, photomultiplier tubes, and inorganic photodiodes, which are also hampered by integrability issues. In the past few years, Shinar et al. have demonstrated the efficacy of the OLED-based platform for PL-based sensing of various analytes [7-13]. We believe that integration of organic photodetectors (OPDs) with this - hence an all-organic sensing platform - has the potential to truly enable the development of flexible, thin, miniature sensor arrays via a facile and low-cost fabrication route. There have been only a few reports on the use of OPDs in PL-based sensors. Kraker et al. [14] recently reported a solid-state OLED/dye/OPD sensing system for PL intensity-based detection, requiring polarization filters to prevent the OLED's electroluminescence (EL) from reaching the OPD. Such EL blocking is crucial for the intensity-based detection methodology. Hofmann et al. [15] reported the use of an OPD to monitor a chemiluminescent reaction in a microfluidic system. Here, we report for the first time, the exploration of an OLED/dye/OPD-based sensing system in both PL intensity $(I)$ and decay time $(\tau)$ detection modes. The $\tau$ mode is preferable as it eliminates the need for (i) frequent sensor calibration, since $\tau$ is insensitive to changes in $I$, minor film degradation, or background light $[9,15,16]$ and (ii) optical filters, as $\tau$ is monitored during the off period of the pulsed excitation. 


\section{Results and discussion}

We explored poly(3-hexylthiophene): [6,6]-phenyl-C61-butyric acid methyl ester (P3HT:PCBM)-based bulk-heterojunction type devices as our OPDs due to their solution processibility and superior performance in the area of photovoltaics $[17,18]$. For quantitation of our OPD's response, oxygen and glucose were chosen as the analytes. The sensing element usually contains an oxygen-sensitive dyes, such as Pt or Pd octaethylporphyrin (PtOEP or PdOEP, respectively) [7-13]. The collisions of the dye molecules with $\mathrm{O}_{2}$ decrease $I$ and $\tau[9,15,16]$. Ideally, in a homogeneous matrix, the $\mathrm{O}_{2}$ concentration can be determined by monitoring $\tau$ or the steady-state $I$ using the Stern-Volmer (SV) equation [19]

$$
I_{o} / I=\tau_{\mathrm{o}} / \tau=1+\mathrm{K}_{\mathrm{SV}}\left[\mathrm{O}_{2}\right]
$$

where $I_{o}$ and $\tau_{\mathrm{o}}$ are the unquenched values and $\mathrm{K}_{\mathrm{SV}}$ is a constant. We used PtOEP in our experiments. It was embedded in $\mathrm{T} \mathrm{TiO}_{2}$ nanoparticle-doped polystyrene film. $\mathrm{TiO}_{2}$ improves EL absorption by PtOEP by increasing scattering within the polystyrene matrix [11]. Both inorganic LEDs and small-molecule OLEDs were utilized as green excitation sources (emission peak $\sim 525 \mathrm{~nm}$ ). The LEDs were operated in a pulsed mode (100 $\mu$ s pulse width at $50 \mathrm{~Hz})$. The PL of the sensing film is in the red region $(\sim 640 \mathrm{~nm})$. Hence, as a first step, the processing of the $\mathrm{P} 3 \mathrm{HT}: \mathrm{PCBM}$ active layer was tailored to improve the photoresponse of these OPDs in the red, which otherwise peaks in the green and is weak in the red $[17,18]$.

In an effort to red-shift the EQE spectrum, three types of OPDs (A, B, and C) with different active layer thicknesses were obtained by spin-coating at 400, 600 and $1000 \mathrm{rpm}$ for 30, 60, and 60 seconds, respectively (see supporting information for device schematic). The 
absorption spectra of these P3HT:PCBM layers are shown in Figure 1a. Device A, because its active layer is thicker $(\sim 350 \mathrm{~nm})$ than those of devices $\mathrm{B}(\sim 220 \mathrm{~nm})$ and $\mathrm{C}(\sim 140 \mathrm{~nm})$, demonstrates the highest absorption at all wavelengths. The three absorption shoulders are more pronounced in device A, indicating a higher degree of self- organization of P3HT chains arising from the slowest drying rate, due to the lower spin speed and duration [17]. This self-organization leads to high crystalline order involving an enhanced conjugation length of P3HT chains $[17,20]$. The EQE spectra for the three devices were measured in short-circuit condition (Figure 1b), and at $0.5 \mathrm{~V}$ reverse bias (Figure 1c). The EQE at short circuit condition for device A shows a maximum of $\sim 70 \%$ at $600 \mathrm{~nm}$, while the peak is at $540 \mathrm{~nm}(\mathrm{EQE} \sim 70 \%$ ) for device B, and $520 \mathrm{~nm}(\mathrm{EQE} \sim 40 \%)$ for device C. The thinner films' thickness (devices B and C) is less than the penetration depth of the strongly absorbed green light, so that the green photons can create a uniform distribution of photogenerated carriers throughout the thickness. But for the thicker film (device A), green photons, having a high absorption coefficient, are absorbed closer to the anode. This makes the electrons more susceptible to recombination, as they have to travel the entire active layer thickness to reach the $\mathrm{Al}$ electrode. In contrast, the red photons can penetrate greater thickness to generate a more uniform carrier distribution. Hence, for device A, the collection efficiency of charge carriers created by red photons is higher than that created by green photons, which explains the $600 \mathrm{~nm}$ EQE peak. The EQE dependence on wavelength does not change with $0.5 \mathrm{~V}$ reverse bias. However, collection at every wavelength improves, enhancing the overall EQE. At PtOEP's emission peak of $640 \mathrm{~nm}$, device A showed the highest EQE of $\sim 40 \%$ at $0 \mathrm{~V}$ and $\sim 50 \%$ at $-0.5 \mathrm{~V}$. In general, photodetectors can be operated at either zero or reverse bias. Operation at zero bias is however advantageous in one aspect, that is, lower dark current 
which assures a high dynamic range. For device A, the dark current was less than $1 \mathrm{nA} / \mathrm{cm}^{2}$, leading to a dynamic range exceeding $10^{7}$ (see supporting information).

To elucidate the structural properties of the P3HT:PCBM films, atomic force microscopy (AFM) was employed. Height AFM images (Figure 2) show that the surface r.m.s. roughness values, $\sigma$, for films $\mathrm{A}$ and $\mathrm{B}$ are $10.7 \mathrm{~nm}$ and $7.2 \mathrm{~nm}$, respectively. For film $\mathrm{C}$, the smoothest surface, with $\sigma \sim 1.05 \mathrm{~nm}$, is observed. The high surface roughness of slowly spin-coated films A and B is another signature of polymer (blend) self-organization, and can be correlated to formation of nanocrystallites due to ordering and stacking of P3HT supermolecules [17]. Raman spectra also show narrowing of the peak related to $-\mathrm{C}=\mathrm{C}-$ symmetric stretching in the active layer of device A, which indicates higher P3HT crystallinity (see supporting information) [21-24]. Higher P3HT crystallinity involves enhanced conjugation length, which leads to enhanced absorption in the red. This, in addition to greater film thickness, can also be partially responsible for enhanced red EQE in device A.

Due to enhanced EQE in the red, device A was chosen as the OPD for our sensors. The OPD, assembled with the sensing film and a $600 \mathrm{~nm}$ long-pass filter, was first tested for $\mathrm{O}_{2}$ sensing using the inorganic LED with peak emission at $\sim 525 \mathrm{~nm}$. In another experiment, an OLED was used. As a first step towards structural integration, the LED, PS:PtOEP sensing film, long pass filter, and P3HT:PCBM OPD were assembled in the front detection geometry (see supporting information for schematic). The filter was placed between the OPD and the sensing film to prevent the green EL from reaching the OPD. Note that the filter is required only for the $I$ detection mode. The $\tau$ mode does not require it, since measurements are done following the excitation pulse, i.e. in the (O)LED's off state. 
Fig. 3 shows the OPD response to the sensor's PL following the LED excitation pulse and exposure to different concentrations of $\mathrm{O}_{2}$ in Argon. As expected, $I$ and $\tau$ decrease with increasing $\mathrm{O}_{2}$ concentration due to collisional quenching, to which the OPD responds with a reduced photocurrent and its faster decay. This PL quenching of PtOEP by $\mathrm{O}_{2}$ is due to the paramagnetic triplet nature of ground state $\mathrm{O}_{2}$ and singlet nature of excited $\mathrm{O}_{2}$ [16], which is unique among common gases. Figure $3 \mathrm{~b}$ shows $I_{o} / I\left(I_{o}\right.$ is the intensity in $100 \%$ Ar) versus the gas-phase $\mathrm{O}_{2}$ concentration. The dependence was found to be linear with $\mathrm{O}_{2}$ concentration up to $40 \% \mathrm{O}_{2}$, with signal ratio $\mathrm{S} \equiv I_{o} / I\left(40 \% \mathrm{O}_{2}\right) \sim 10$, which can be further improved by using a $630 \mathrm{~nm}$ long-pass filter. The SV curve for the $\tau$ mode shows that $\tau_{o} / \tau$ for $20 \%$ oxygen is 2.5 , which is lower than the $I$ mode ratio. However, other advantages associated with the $\tau$ mode, as discussed earlier, make it more viable for practical applications. The deviation of the $\tau$ mode SV plot from linearity probably arises from inhomogeneity in the dye's environment, i.e. the dye molecules occupy quencher-easy accessible and quencher-difficult accessible sites [25], which leads to different contributions to PL quenching. However, the exact mechanism is not clear at this point.

Glucose sensing using the LED/PS:PtOEP sensing element/P3HT:PCBM OPD configuration relied on the enzymatic oxidation of glucose by glucose oxidase $\left(\mathrm{GO}_{\mathrm{x}}\right)$ and oxygen. In the presence of glucose and $\mathrm{GO}_{\mathrm{x}}$, the PL quenching of the dye molecules is reduced due to consumption of dissolved oxygen (DO). The DO's initial concentration ([DO $]_{\text {initial }}$ ) in water is $\sim 0.26 \mathrm{mM}$ at room temperature. For a concentration of the active isomer of glucose $\left([\beta-\mathrm{D} \text {-glucose }]_{\text {initial }}\right)<[\mathrm{DO}]_{\text {initial }}$, at the completion of the oxidation reaction, $[\beta \text {-D-glucose }]_{\text {initial }}$ equals the difference between the initial and final DO levels. With 
increased [ $\beta$-D-glucose $]_{\text {initial }}$, the residual DO decreases, hence the $I$ and $\tau$ of PtOEP increase (Figure $3 \mathrm{c}, \mathrm{d}$ ). Integration under the PL $\tau$ curve, corresponding to each $[\beta-\mathrm{D}-\text { glucose }]_{\text {initial }}$, was used to represent the $I$. It can be seen that $1 / I$ is linear with $[\beta-D \text {-glucose }]_{\text {initial }}$, which is expected from a modified SV equation [12]. The $\tau$ mode curves slightly deviate from linearity similar to the case of the gas-phase $\mathrm{O}_{2}$.

Finally, following the demonstration of the suitability of the OPD for $\mathrm{O}_{2}$ and glucose sensing, including in the $\tau$ mode, the inorganic LED was replaced by an OLED to demonstrate the viability of an all-organic sensor platform. In this experiment, $I$ and $\tau$ clearly decrease as the $\mathrm{O}_{2}$ concentration is increased, as expected (Figure 3e, f). The observed higher noise in the photocurrent decay curves is due to instabilities in the EL and lower brightness than the inorganic LED. $I_{d} / I$ is linear with $\mathrm{O}_{2}$ concentration and the ratio $I_{d} / I$ for $15 \%$ oxygen is 2.1. Although this ratio should be the same whether using an LED or OLED, it has previously been shown that weaker excitation by the OLED generally results in a lower ratio. ${ }^{[10,13]}$ The results with the OLEDs can therefore be improved by utilizing brighter (and encapsulated) OLEDs. The ratio $\tau_{o} / \tau$ for $15 \%$ oxygen is 1.6 and is comparable to the value observed when using the inorganic LED, since performance in the $\tau$ mode is independent of the intensity of excitation source.

\section{Conclusion}

In summary, a structurally integrated all organic sensing platform - OLED pixels exciting a luminescent dye; the dye's PL intensity and decay-time depending on an analyte's concentration; and these PL changes of the dye being detected by OPDs - is a promising approach to achieve low-cost, flexible and compact sensor arrays. This communication 
presented steps towards realizing this paradigm in one the several possible embodiments - a front detection geometry, wherein, the (O)LED, the dye embedded film, and the OPD were spatially assembled in the same order. We engineered the P3HT:PCBM OPDs to tailor their

photoresponse towards the red emitting dye (PtOEP) based $\mathrm{O}_{2}$ and glucose sensors. Devices realized from a thicker and slower-grown P3HT:PCBM layer showed the highest EQE of 40\% without bias at $640 \mathrm{~nm}$, which is the peak emission of the sensing dye. Oxygen and glucose were monitored using the optimized OPD via detection of the dye's $I$ or $\tau$. The latter eliminates the need for frequent sensor calibration or optical filters. The response of the OPDs was sufficiently fast to monitor the $\mathrm{O}_{2}$ using the $\tau$ mode. Finally, after demonstrating the efficacy of OPDs with inorganic LEDs, this report also demonstrated all-organic $\mathrm{O}_{2}$ sensors, which, in addition to OPDs, used OLEDs as the light source.

\section{Experimental}

OPD fabrication and characterization: For OPD fabrication, a conducting film of poly(3,4-ethylenedioxythiophene) doped with poly(styrenesulfonate) (PEDOT:PSS, Clevios P) was spin coated at $3000 \mathrm{rpm}$ after UV-Ozone plasma exposure of cleaned ITO-coated slides, followed by annealing at $120{ }^{\circ} \mathrm{C}$ for 5 minutes. The P3HT:PCBM blend solution (17 $\mathrm{mg} / \mathrm{ml}$ in dichlorobenzene) was spin coated at different speeds. An Al (100 nm) electrode was deposited by thermal evaporation on top of the active layer. The absorption spectra were measured by a Varian Cary 5000 UV-Vis-NIR spectrophotometer. EQE measurements were done using ELH Quartzline lamp (120V-300W from GE) and a monochromator with a lockin amplifier to eliminate background noise. The reference was a calibrated Si photodiode with known EQE spectra. The P3HT:PCBM layer thicknesses were obtained by forming a 
$100 \mu \mathrm{m}$ wide scratch on the films using a fine blade. AFM (Veeco Nanoscope III) tip in tapping mode was scanned across the scratch to find the thickness of the P3HT:PCBM films. Raman spectra were recorded on a Renishaw inVia Raman microscope equipped with a low noise and high sensitivity RenCam CCD detector, and a $488 \mathrm{~nm}, 0.3 \mathrm{~mW}$ laser. The reflected Raman signal was collected using a 50X objective with a numerical aperture of 0.7 . The signal collection time was $10 \mathrm{~s}$ and the scan was averaged twice. To mimic the device fabrication conditions, all the films for absorption and Raman spectra measurement were spun cast on PEDOT:PSS-covered ITO-coated glass substrates.

OLED Fabrication: $20 \Omega / \square$ ITO/glass was obtained from Colorado Concept Coatings. Copper phthalocyanine $(\mathrm{CuPc})$ and $\mathrm{LiF}$ were obtained from Sigma-Aldrich. N,N'-diphenylN,N'-bis(1-naphthyl phenyl)-1,1 '-biphenyl-4,4'-diamine (NPD), 2,3,6,7-Tetrahydro-1,1,7,7,tetramethyl- 1H,5H,11H-10-(2-benzothiazolyl) quinolizino-[9,9a,1gh] coumarin (C545T), and tris(8-hydroxyquinoline) $\mathrm{Al}\left(\mathrm{Alq}_{3}\right)$ were obtained from H.W. Sands. Green emitting (peaking at $\sim 525 \mathrm{~nm}$ ) OLED pixels were fabricated by thermally evaporating organic materials on top of $\sim 150 \mathrm{~nm}$ thick cleaned and UV ozone-treated ITO-coated glass. The organic layers, in sequence, are the hole injection layer $\sim 5 \mathrm{~nm} \mathrm{CuPC}$, hole transport layer

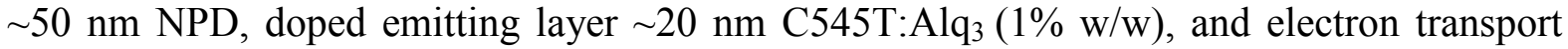
layer $\sim 30 \mathrm{~nm} \mathrm{Alq}_{3}$, which is followed by an electron injection layer $\sim 1 \mathrm{~nm} \mathrm{LiF}$ and the $\sim 100$ $\mathrm{nm}$ Al cathode. OLED pixels were generated by etching the ITO into two $2 \mathrm{~mm}$ wide strips; the OLED pixels are defined by the overlapping regions of mutually perpendicular ITO and Al strips. Two OLED pixels $(2 \mathrm{~mm} \times 2 \mathrm{~mm})$ were used as the excitation source for the PL measurements. 
Sensing Experiment: PS:PtOEP sensor films were prepared by drop casting $50 \mu \mathrm{L}$ of a toluene solution with $1 \mathrm{mg} / \mathrm{mL}$ PtOEP, $1 \mathrm{mg} / \mathrm{mL} \mathrm{TiO}_{2}$ and $40 \mathrm{mg} / \mathrm{mL}$ polystyrene. The films were dried in the dark at ambient temperature. GOx from Aspergillus niger was obtained from Sigma-Aldrich. GOx and glucose (Fisher Scientific) were dissolved in phosphate buffer (PH 7.4), at the desired concentrations. The sensor components - an LED, PS:PtOEP sensing film, long-pass filter, and P3HT:PCBM OPD - were assembled in a front detection mode, where the sensing film is sandwiched between the OPD and LED. For $\mathrm{O}_{2}$ sensing experiments, the sensor film was enclosed in a flow cell through which different volumetric ratios of $\mathrm{Ar} / \mathrm{O}_{2}$ mixture gas were passed. The inorganic LEDs were operated in a pulsed mode at a bias of $3.7 \mathrm{~V}$, pulse width of $100 \mu \mathrm{s}$, and a repetition rate of $50 \mathrm{~Hz}$. The photocurrent signal from the OPD at zero bias was amplified using a gain of $10^{6} \mathrm{~V} / \mathrm{A}$ at 200 $\mathrm{kHz}$ bandwidth, and monitored on an oscilloscope. The PL lifetimes were obtained by monitoring the OPD response following the application of the LED pulse. For glucose sensing, a glass tube was glued on top of the sensor film, forming a reaction well $(200 \mu \mathrm{L}$ in volume), enclosing the dye- coated film at the bottom. $100 \mu \mathrm{L}$ of glucose and $\mathrm{GO}_{\mathrm{x}}$ were sequentially added into the reaction well, followed by hermetic sealing using a cover glass. The PL signal was collected by the OPD after 1 minute of adding the solutions. The concentration of $\mathrm{GO}_{\mathrm{x}}(300$ units $/ \mathrm{mL})$ was sufficient to catalyze glucose oxidation in the range of $0 \sim 0.3 \mathrm{mM}$, deplete the $\mathrm{DO}$ in $20 \mathrm{sec}$.

\section{Acknowledgements}

SC, KSN and AL thank the Institute of Physical Research and Technology, Iowa State University for Company Assistance grant. SC, RS and JS thank the Iowa Power Fund. 
JS and YC thank the Ames Laboratory, which is operated by Iowa State University for the US Department of Energy (USDOE) under Contract No. DE-AC 02-07CH11358. The work was partially supported by the Director for Energy Research, Office of Basic Energy

Sciences, USDOE. RS and Integrated Sensor Technologies thank NSF for an SBIR Phase II grant.

\section{References}

[1] H-Y. Chen, J. Hou, S. Zhang, Y. Liang, G. Yang, Y. Yang, L. Yu, Y. Wu, G. Li, Nat. Photon., 3 (2009) 649.

[2] K. M. Vaeth, Inform. Display, 19 (2003) 12.

[3] X. Gong, M. Tong, Y. Xia, W. Cai, J. S. Moon, Y. Cao, G. Yu, C-L. Shieh, B. Nilsson, A. J. Heeger, Science, 325 (2009) 1665.

[4] J. Shinar, R. Shinar, J. Phys. D: Appl. Phys., 41 (2008) 133001.

[5] O. Hofmann, X. Wang, A. Cornwell, S. Beecher, A. Raja, D. D. C. Bradley, A. J. deMello, J. C. deMello, Lab Chip, 6 (2006) 981.

[6] E. J. Cho, F. V. Bright, Anal. Chem., 73 (2001) 3289.

[7] V. Savvate'ev, Z. Chen-Esterlit, J. W. Aylott, B. Choudhury, C. H. Kim, L. Zou, J. H. Friedl, R. Shinar, J. Shinar, R. Kopelman, Appl. Phys. Lett., 81 (2002) 4652.

[8] B. Choudhury, R. Shinar, J. Shinar, J. Appl. Phys., 96 (2004) 2949.

[9] R. Shinar, Z. Zhou, B. Choudhury, J. Shinar, Anal. Chim. Acta, 568 (2006) 190.

[10] R. Shinar, D. Ghosh, B. Choudhury, M. Noack, V. L. Dalal, J. Shinar, J. Non-Cryst. Solids, 352 (2006) 1995.

[11] Z. Zhou, R. Shinar, A. J. Allison, J. Shinar, Adv. Funct. Mater., 17 (2007) 3530.

[12] Y. Cai, R. Shinar, Z. Zhou, J. Shinar, Sensors Actuators B, 134 (2008) 727.

[13] D. Ghosh, R. Shinar, V. Dalal, Z. Zhou, J. Shinar, J. Non Cryst. Solids, 354 (2008) 2606. 
[14] E. Kraker, A. Haase, B. Lamprecht, G. Jakopic, Appl. Phys. Lett. 92 (2008) 033302.

[15] O. S. Wolfbeis, (Ed.), Fiber Optic Chemical Sensors and Biosensors, CRC Press, BocaRaton, FL, 1991.

[16] Y. Amao, Michrochim. Acta, 143 (2003) 1.

[17] G. Li, V. Shrotriya, J. Huang, Y. Yao, T. Moriarty, K. Emery, Y. Yang, Nat. Mater., 4 (2005) 864.

[18] J. Y. Kim, S. H. Kim, H. H. Lee, K. Lee, W. Ma, X. Gong, A. J. Heeger, Adv. Mater., 18 (2006) 572.

[19] J. R. Lakowicz, Principles of Fluorescence Spectroscopy, Plenum Press, New York (1983).

[20] M. Sunderberg, O. Inganas, S. Stafstrom, G. Gustafsson, B. Sjogren, Solid State Commun., 71 (1989) 435.

[21] J. Casado, R. G. Hicks, V. Hernandez, D. J. T. Myles, M. C. R. Delgado, J. T. L. Navarrete, J. Chem. Phys., 118 (2003) 1912.

[22] Y. W. Goh, Y. F. Lu, Z. M. Rem, T. C. Chong, Appl. Phys. A: Mater. Sci. Process., 77 (2003) 433.

[23] P. S. O. Patrico, H. D. R. Calado, F. A. C. de Oliveira, A. Righi, B. R. A. Neves, G. G. Silva, L. A. Cury, J. Phys.: Condens. Matter., 18 (2006) 7529.

[24] E. Klimov, W. Li, X. Yang, G. G. Hoffmann, J. Loos, Macromolecules, 39 (2006) 4493.

[25] S. Lee, I. Okura, Spectrochim. Acta Part A, 54 (1998) 91. 


\section{Figures}
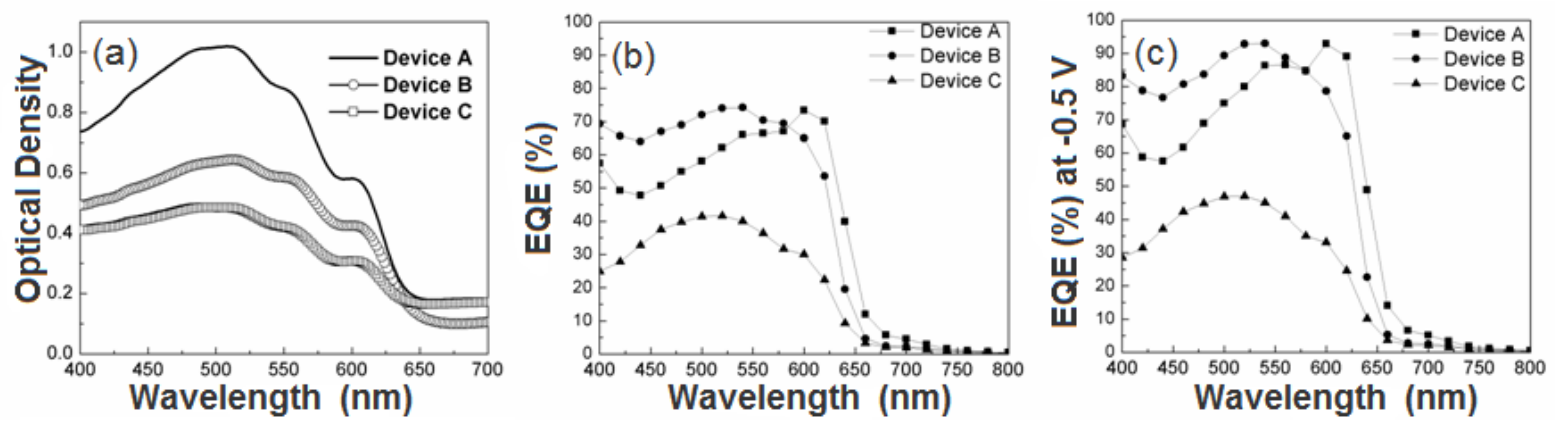

Fig. 1. Effect of active layer growth conditions. (a) UV-Vis absorption spectra for films of P3HT/PCBM (1:1 wt/wt ratio), spin coated at $400 \mathrm{rpm}$ for 30 seconds - Device A, $600 \mathrm{rpm}$ for 60 seconds - Device B, and $1000 \mathrm{rpm}$ for 60 second - Device C. (b) EQE spectra of devices $\mathrm{A}, \mathrm{B}$, and $\mathrm{C}$ at short circuit; and (c) at $0.5 \mathrm{~V}$ reverse bias. 

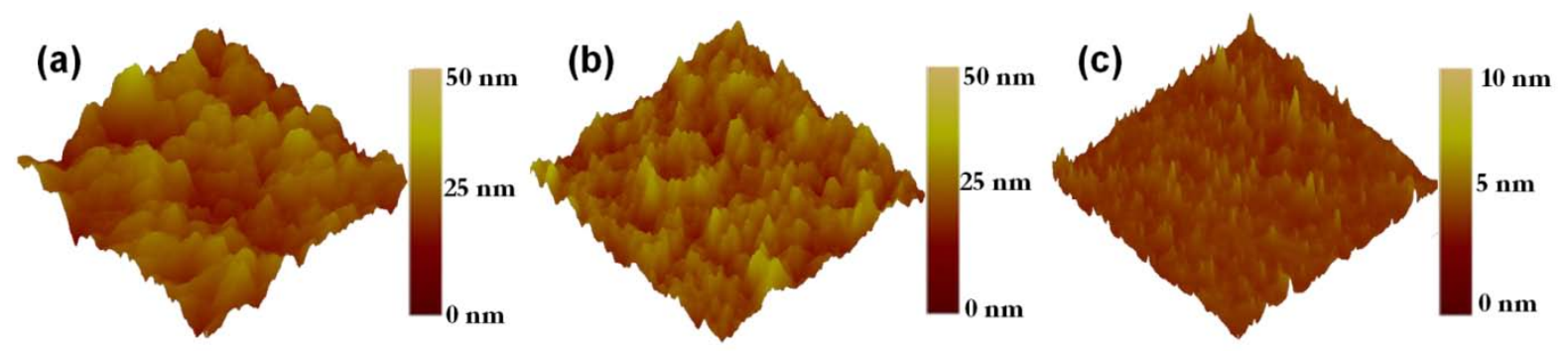

Fig. 2. AFM height images of the P3HT/PCBM composite films (PCBM concentration $=50$ $\mathrm{wt} \%$ ) showing the active layer of (a) device A (b) device B and (c) device C. Scan area is 5 $\mu \mathrm{m} \times 5 \mu \mathrm{m}$ in all cases. Note that the color scale for films $A$ and $B$ is $0-50 \mathrm{~nm}$, whereas for film $\mathrm{C}$ it is $0-10 \mathrm{~nm}$. 

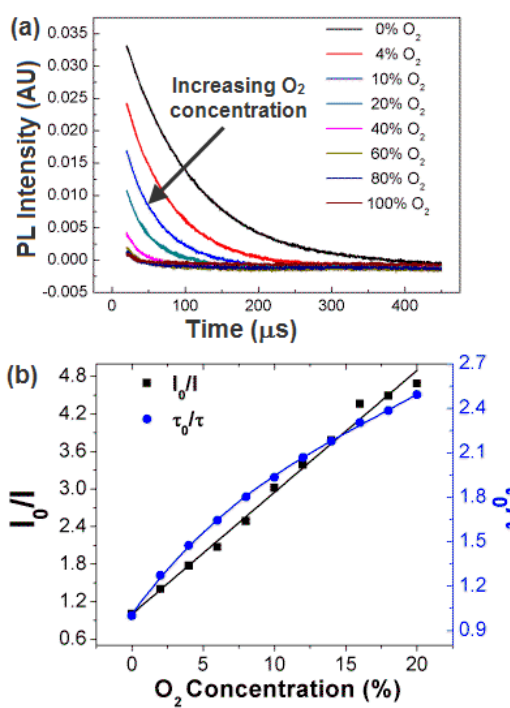

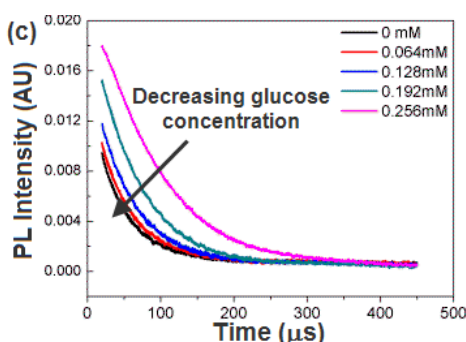

(d)

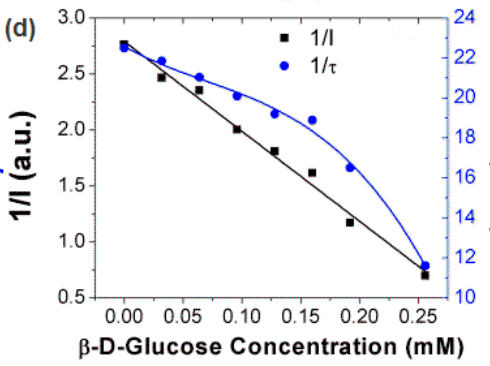

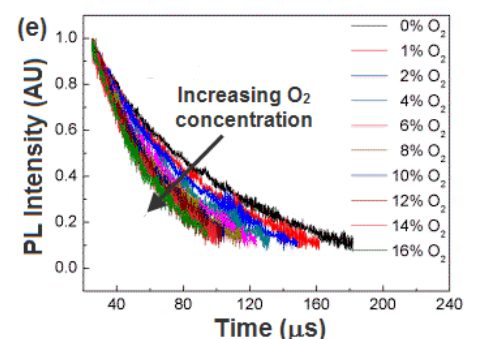

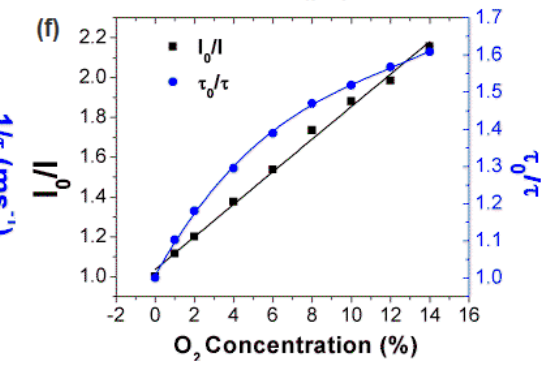

Fig. 3. The effect of concentration of gas-phase $\mathrm{O}_{2}$ (a) and glucose (b) on the OPD's temporal photocurrent response. Excitation source was an LED. (b) and (d) are $I$ and $\tau$-based SV calibration curves corresponding to (a) and (c), respectivly. For OLED excited $\mathrm{O}_{2}$ sensor, (e) and (f) show the effect of $\mathrm{O}_{2}$ concentration on the OPD temporal response and corresponding SV calibration curves, respectively. 


\section{Chapter 8. Summary}

General introduction to OLED basics and OLED-based structurally integrated sensors was provided in chapter 1 and chapter 2. As discussed in chapter 3, OLEDs were developed or improved using novel engineering methods for better charge injection (increased by over 1 order of magnitude) and efficiency. As the excitation sources, these OLEDs have preferred characteristics for sensor applications, including narrowed emission, emission at desired wavelength, and enhanced output for reduced EL background, higher absorption and improved device lifetime.

In addition to OLEDs with desired performance, sensor integration requires oxidase immobilization with the sensor film for $\mathrm{O}_{2}$-based biological and chemical sensing. Nanoparticles such as $\mathrm{ZnO}$ have large surface area and high isoelectric point $(\sim 9.5)$, which favors enzyme immobilization via physical adsorption as well as Coulombic bonding. In chapter 4, it was demonstrated that $\mathrm{ZnO}$ could be used for this purpose, although future work is needed to further bond the $\mathrm{ZnO}$ to the sensor film.

In chapter 5, single unit sensor was extended to multianalyte parallel sensing based on an OLED platform, which is compact and integrated with silicon photodiodes and electronics. Lactate and glucose were simultaneously monitored with a low limit of detection $0.02 \mathrm{mM}$, fast response time ( $\sim 1$ minute) and dynamic range from 0-8.6 ppm of dissolved oxygen. As discovered in previous work, the dynamic range covers $0-100 \%$ gas phase $\mathrm{O}_{2}$ or $0-40 \mathrm{ppm}$ dissolved oxygen at room temperature. 
PL decay curve, which is used to extract the decay time, is usually not a simple exponential at high $\mathrm{O}_{2}$ concentration, which indicates that $\mathrm{O}_{2}$ is not equally accessible for different luminescent sites. This creates a challenge for data analysis, which however was successfully processed by stretched exponential as shown in chapter 6 . This also provides an insight about the distribution of $\mathrm{O}_{2}$ :dye collisional quenching rate due to microheterogeneity. Effect of $\mathrm{TiO}_{2}$ doping was also discussed. Stretched exponential analysis also generates calibration curves with higher sensitivity, which is preferred from the operational point of view.

The work of enhanced integration was shown in chapter 7 with a polymer photodetector, which enables the preferred operation mode, decay time measurement, due to fast reponse $(<20 \mu \mathrm{s})$. Device thickness was enlarged for maximum absorption of the PL, which was realized by slow spincoating rate and shorter spincoating time. Film prepared this way shows more crystalline order by Raman spectra, probably due to slow evaporation. This also ensures charge transport is not affected even with a thick film as indicated in the response time. Combination of OLEDs and polymer photodetectors present opportunities for solution processed all-organic sensors, which enables cheap processing at large scale.

Future development can focus on monolithically integration of OLEDs and organic photodetectors (OPD) on the same substrate at a small scale, which could be enabled by inkjet printing. As OLED and OPD technologies continue to advance, small-sized, flexible and all-organic structurally integrated sensor platforms will become true in the near future. 


\section{Acknowledgements}

I would like to express my deep gratitude to my advisors Dr. Joseph Shinar and Dr. Ruth Shinar for all the years' the guidance, encouragement, support, and help, without which the work presented in this dissertation would not have been possible.

I would like to thank my parents and my lovely and beautiful wife who are always standing behind me and supporting me. It's their selfless love that has brought me this far. I also want to dedicate this dissertation to my father, who is my most important mentor and friend throughout my life. His influence made me a strong person, who will never stop striving for excellence and never give up without trying hard. Carrying his love and care, I feel confident no matter what challenges I am facing.

Special thanks to my colleagues, Yun Tian, Zhaoqun Zhou, Zhengqing Gan, Rui Liu, Alex Smith, Ying Cheng, et al. Discussions and work with them stimulated many interesting ideas and made my understanding deeper and broader.

Many thanks to the funding agencies, including US Department of Energy, NASA, and NSF etc. I would also like to thank Larry, Lori, Gloria, Diane and Deb for their administrative work. 